UCRL-53295

Distribution Category UC-20, 20a,

20d, 20f, $20 \mathrm{~g}$

UCRL -53295

DE8 2020610

\title{
A Study of Impurities in the Tandem Mirror Experiment Using Extreme-Ultraviolet Spectroscopy
}

\author{
O. T. Strand
}

Manuscript Date: May 12, 1982

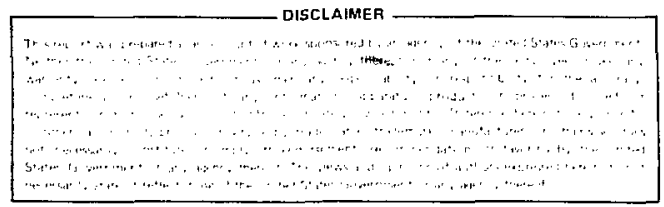

LAWRENCE LIVERMORE LABORATORY

University of California $\bullet$ Livermore, California $\bullet 94550$ 
ABSTRACT

Impurities in the Tandem Mirror Experiment (TMX) have been siudied using extreme ultraviolet spectroscopy. Three time-resolving absolutely-calibrated normal-incidence monochromators, one on each section of TMX, were used to study the impurity emissions in the wavelength range of $300 \AA-1600 \AA$. The instruments on the east end cell and central cell were each capable of obtaining spatially-resolved profiles from 22 chords of the plasma simultaneously while the instrument on the west end cell monitored the central chord. The impurities identified in TMX were carbon, nitrogen, oxygen, and titanium. Emphasis was placed upon determining the impurity densities and radiated power losses of the central cell; results indicate that the impurity concentrations were low--less than $0.4 \%$ for each species--and that less than $10 \%$ of the total net trapped neutral beam power was lost to radiation. The use of titanium gettering on the central cell walls was observed to decrease the brightnesses of singly- and doubly-ionized carbon and oxygen in the central ceil plasma. In the end cells, oxygen was the main impurity with a concentration of about $1.5 \%$ and was injected by the neutral beams; the other impurities had concentrations of about $0.5 \%$. Radiated losses from the end cells were negligible.

Evidence has been obtained which indicates that impurities in the central cell are subject to a radially outward transport. The density ratios of $0 \mathrm{~V}$ to 0 VI indicate a short $(0.3-3 \mathrm{~ms})$ central cell confinement time for $0 \mathrm{VI}$ ions, even though calculations predict that axial confinement times should be longer than the TMX shot duration. Impurity puffing experiments performed in the central cell of TMX support this conclusion. Oxygen and neon puffed into the central cell during the TMX shot were observed to accurmulate in the outer boundary of the plasma, while only a small fraction penetrated into the central regions. None of the injected impurity was observed in the end cell plasmas; instrumental sensitivity puts an upper limit of 0.03 on the ratio of injected impurity density in the end cells to that in the central cell. 0xygen puffed before the TMX shot was observed to decay from all rogions of the plasma during the shot. These results provide an explanation for the observed low concentrations of impurities in the central cell of TMX. 
ACKNOWLEDGMENTS

This report originated as a Ph.D. dissertation submitted to the Johns Hopkins University in Baltimore, MD. The work performed for this study is the result of a collaboration between Johns Hopkins University (JHU) and the Lawrence Livermore National Laboratory (LLNL); therefore, there were two groups of pecple involved in the successful completion of this project. At JHU, H. W. Moos is the head of the plasma spectroscopy group; the state-of-the-art spatial-imaging monochromators designed and built by his group made possible the undertaking of this research. His own interest in the project and his perception and skill as a plasma spectroscopist contributed much toward understanding and analyzing the data. Other members of the group at JHU contributed to this work. E. Tracy spent the summer of 1980 in Livermore and endured many long hours helping to take data. R. E. Bell wrote the software for the 3-D computer plots. J. Mintor and W. Ruff assisted in the absolute calibration of the instruments at JHU. P. Coffman and M. Neikirk kept the coast-to-coast lines of communication and support open throughout the project.

At LLNL, the list of people who took an active part in this project is almost endless. The project leader was F. H. Coensgen who was always in full support of this effort. T. C. Simonen always showed an interest in the data and its interpretation. R. S. Hornady initiated the collaboration between JHU and LLNL on the 2XIIB experiment and provided the framework for this project also. S. L. Allen was originally at JHU before he joined the TMX group and provided an immense amount of help with taking and analyzing data, computer modeling and writing journals. R. P. Drake provided the driving force necessary to perform the impurity puffing experiments. All other members of the TMX group readily assisted as much as possible, including T. A. Casper, J. F. Clauser, D. L. Correll, W. F. Cummins, J. C. Davis, J. H. Foote, A. H. Futch, R. K. Goodman, D. P. Grubb, E. B. Hooper, A. L. Hunt, A. W. Molvik, R. H. Munger, W. E. Nexsen, T. J. Orzechowski, W. L. Pickles, P. Poulsen, and B. W. Stallard. Technical assistance was provided by G. H. Cobb, J. J. Jolly, G. E. Mattson, and J. J. Murphy. C. R. Perry and her secretarial staff were always cheerfully helping with everything from typing to unraveling red tape. Martelle Mays did an excellent job of typing this manuscript. 
TABLE GF CONTENTS

I. Introduction

II. The Tandem Mirror Experiment (TMX)

1. Introduction

2. Enhanced Confinement Times in TMX

3. TMX Parameters

4. Major Results from TMX

5. TMX Upgrade

III. EUV Plasma Spectroscopy on TMX

1. Introduction

2. Brightnesses and Ion Densities

3. Ion Density Radial Profile Models and Concentrations

4. Radiated Power Losses

5. Doppler Corrections

5.1 Doppler-Broadened Singlet

5.2 Doppler-Broadened Multiplet

IV. Description of the Spectroscopic Equipment

1. Introduction

2. The Single-Channel Instrument

3. The Spatial-Imaging Monochromators

4. Instrument Locations on TMX

v. Intrinsic Impurity Brightnesses and Concentrations

1. Introduction

2. General Characteristics of Impurity Emissions

2.1 Impurities Identified

2.2 Temporal Profiles

2.3 Spatial Profiles

2.4 Doppler Widths of EUV Emissions $\quad 54$

3. Impurity Concentrations in TMX 58

3.1 Light Impurities $\quad 58$

3.1.1 Method 58

3.1.2 Metastable Densities $\quad 58$

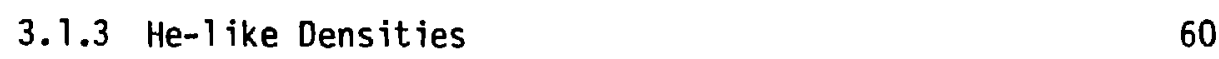

3.1.4 High Electron Temperature at Edge of Central Cell Plasma $\quad 60$ 
TABLE OF CONTENTS (continued)

3.2 Titanium

3.2.1 Method

3.2.2 Excitation Rates

64

3.2.3 Ionization Equilibrium

64

3.2.4 Results

4. Radiated Power Losses from TMX 66

5. Sources of Impurities 68

5.1 End Cell Sources 68

5.2 Central Cell Sources $\quad 72$

$V I$. Confinement Times of Intrinsic Impurities in the Central Cell of TMX

$\begin{array}{ll}\text { 1. Introduction } & 74\end{array}$

2. Predicted Axial Confinement Time of Impurities 74

3. Experimental 0 VI Confinement Time 76

4. Conf inement Modified Coronal Equilibrium 79

5. Steady-State Model for Oxygen Ions $\quad 81$

5.1 Introduction $\quad 81$

$\begin{array}{ll}5.2 \text { Description of Model } & 81\end{array}$

5.3 General Trends 83

5.4 Discussion of Results $\quad 84$

VII. Impurity Puffing Experiments in the Central Cell of TMX 88

1. Introduction 88

2. Experimental Procedure 88

3. Late Puffing Experiments $\quad 90$

$\begin{array}{ll}3.1 \text { Late Puffing-0xygen } & 90\end{array}$

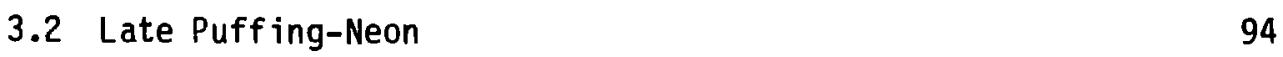

3.3 Late Puffing - End Cell Results 97

4. Early Puffing - Oxygen 97

5. Discussion 99 
TABLE OF CONTENTS (continued)

$\underline{\text { Page }}$

Appendices

A. Rate Coefficients

101

A.1 Electron Impact Excitation Rates 101

A.2 Electron Impact Ionization Rates 101

A.3 Radiative and Dielectric Recombination Rates 103

B. Flowchart of Data Acquisition, Display, and Storage 113

C. Details of the Spatial-Imaging Monochromators 115

$\begin{array}{lll}\text { C. } 1 \text { The Livermore SIDS (LLNL-SIDS) } & 115\end{array}$

C.1.1 The $0.4 \mathrm{~m}$ Normal Incidence Monochromator 115

C.1.2 The Spatial Imaging Detector System (SIDS) 116

C.1.3 Calibration Results of the LLNL-SIDS 118

C.2 The Johns Hopk ins University SIDS (JHU-SIDS) 127

C.3 The Calibration Procedure 131

$\begin{array}{ll}\text { References } & 136\end{array}$ 
Fig. II.l. (a) Simple magnetic mirror showing increased field strength of each end which tends to reflect charged particles.

(b) Loss region in velocity space of an open-ended mirror device.

Fig. II.2. Magnetic field, electron density, and plasma potential along the length of TMX.

Fig. II.3. Timing sequence for typical TMX operation. The gas feed could be via either the gas boxes or puffer valves.

Fig. II.4. Schematic of TMX showing geometry of the magnet

system, neutral beams, start-up guns and magnetic

flux bundle.

Fig. II.5. Comparison of plasma-potential profiles: (a) basic tandem potential profile (TMX), and (b) potential profile with thermal barriers (TMX Upgrade). $\phi_{C}$ $=$ confinement potential, $\phi_{e}=$ central cell potential, and $\phi_{b}=$ potential depression at the barriers.

Fig. IV.1. Schematic diagram of the single channel instrument.

Fig. IV.2. Photometric calibration vs wavelength for

the single channel instrument.

Fig. IV.3. Ray diagram of spatial imaging optics in the LLNL-SIDS instrument.

Fig. IV.4. Final locations of the three EUV instruments on TMX.

Fig. V.1. Temporaliy and spatially resolved brightness of deuterium $L y_{\alpha} 1215 \AA$ from the east end cell of TMX. Note the effect of the start-up gun plasmas.

Fig. V.2. Temporal profiles of oxygen ion brightnesses from the central cell and east end cell of TMX. These are central chord profiles which do not include the effects of the start-up gun plasmas.

Fig. V.3. Central cell radial brightness profiles of 0 II - O VI and the corresponding electrnn line density profiles during gas box fueling of the central cell plasma. 
Fig. V.4. Central-cell radial brightness profiles of N II, density profiles during puffer fueling of the central cell plasma.

Fig. V.5. Central-cell radial profile of (a) deuterium

$L_{\alpha} 1215 \AA$ brightness and (b) the corresponding neutral density.

Fig. V.6. Spectral scan of 0 VI doublet at $1032 \AA$ and 1038 \& from TMX central cell. The line widths are determined by the $2 \AA$ instrumental resolution.

Fig. V.7. Spectral scans from TMX east end cell showing

Doppler broadening of (a) $0 \mathrm{~V} 630 \AA$ and

(b) N II $917 \AA$. The instrumental resolution is $1 \AA$.

Fig. V.8. Brightness profile of Ti IV $779 \AA$ in the east end cell when only the start-up plasma guns are fired. This indicates that the start-up guns are a source of titanium in the end cells.

Fig. VI.1. Predicted confinement time of 0 VI vs electron temperature and density ratio of $0 \mathrm{~V}$ to $0 \mathrm{VI}$. Experimentally obtained values indicate a range in the 0 VI conf inement time of 0.3 to $3 \mathrm{~ms}$.

Fig. VI.2. Calculated oxygen ionization equilibrium for

(a) nearly infinite confinement time and (b) $1 \mathrm{~ms}$ confinement time.

Fig. VI.3. "Reasonable" profiles of (a) electron density,

(b) electron temperature, (c) conf inement time, and (d) neutral oxygen density during gas box operation.

Fig. VI.4. Brightness profiles predicted by the model using

"reasonable" input parameters during gas box operation. Actual brightness profiles are normalized to the code results for comparison.

Fig. VII.1. (a) Unsmoothed and (b) Smoothed radial and temporal profile of total puffed oxygen density in the central cell.

Fig. VII.2. Radial profile of total puffed oxygen density puffing experiments. 
Fig. VII.3. Temporal profiles of total puffed oxygen density at $38 \mathrm{~cm}, 30 \mathrm{~cm}, 20 \mathrm{~cm}$ during the late puffing experiments.

Fig. VII.4. Penetration rate of total puffed oxygen density in the central cell during the late puffing experiments.

Fig. VII.5. Radial profile of puffed (a) Ne II and (b) 0 II 96 density in the central cell at 25 ms.

Fig. VII.6. Temporal profiles of injected 0 II, 0 IV, 0 VI central chord brightness during early puffing experiments.

Fig. A.1. Excitation rates for transitions of carbon ions (Gaunt factors by Davis [42]).

Fig. A.2. Excitation rates for transitions of nitrogen ions (Gaunt. factors by Davis [42]).

Fig. A.3. Excitation rates for transitions of oxygen ions (Gaunt factors by Davis [42]).

Fig. A.4. Excitation rates f,r Lyman alpha, N IV 765 A (LASL), NV 1239 \& (LASL), C IV 1548 \& (LASL).

Fig. A.5. Excitation rates for transitions of oxygen inns (LASL report [39]).

Fig. A.6. Ionization rates for deutei ium and carbon (Lotz $[44])$.

Fig. A.7. Ionization rates for nitrogen and oxygen (Lotz $[44])$.

Fig. A.8. Ionization rates for neon (Lotz [44]).

Fig. A.9. Radiative and dielectronic recomb ination rates for oxygen (Mattioli [46]).

Fig. C.1. Quantum efficiency of the SIDS detector times the transmission of the grating (ur) vs anode in the LLNL-SIDS instrument at $735 \mathrm{~A}$. The effect of the three panels of the grating can be seen.

Fig. r..2. Schematic diagram of the Spatial Imaging Detector System (SIDS). 
Fig. C.3. Photometric calibration vs wavelength for anode

11 of the LLinl-SIDS before and after the TMX experiment.

Fig. C.4. Reflectivity of the center panel of the grating in the LLNL-SIDS vs wavelength order. This grating is blazed at $600 \AA$ in first order.

Fig. C.5. Rejection of scattered 1 ight by the LLNL-SIDS at $584 \AA$. The values are normalized to the signal obtained at $584 \AA$. Note the increase at second order.

Fig. C.6. LLNL-SIDS detector counting rate vs incident photon flux at $584 \AA$. The response of the detector is still linear at $10^{5}$ counts per second per anode.

Fig. C.7. Wavelength calibration of the LLNL-SIDS instrument. The dispersion of the grating results in approximately 30 steps of the wavelength drive per $\AA$.

Fig. C.8. Calibration of the LLNL-SIDS entrance and exit slit widths and entrance slit height. Slit widths of $100 \mu$ corresponds to $I \mathbb{A}$ resolution.

Fig. C.9. LLNL-SIDS detector count rate vs applied high 130 voltage.

Fig. C.10. Photometric calibration vs wavelength for anode 11 of the JHU-SIDS before and after the TMX experiment.

Fig. C.11. Schematic diagram of the extreme ultra-violet calibration equipment (ECE) at Johns Hopkins University. The reference diode has been calibrated at the National Bureau of Standards [17]. 
Table II.1. Typical operating parameters for TMX compared with expected values for TMX-Upgrade.

Table V.1. The typical central-chord brightnesses from the

TMX central cell and east end cell.

Table V.2. Ion energies in east end cell obtained from Doppler broadening measurements

Table V.3. Ratios of metastable to ground state density

$\left(n_{m} / n_{q}\right)$ used to calculate total impurity

densities and radiated power losses.

Table V.4. Ionization state distribution observed for oxygen

in the central cell of TMX.

Table V.5. Average impurity concentrations observed in the TMX central cell and east end cell. The low-impurity concentrations in the central cel1 yield $z_{\text {eff }} \sim 1.1$.

Table V.6. Parameters used to calculate excitation rates for transitions of Si-like and S-like titanium.

Table V.7. Ionization equilibrium distributions used to

calculate total titanium density and radiated power loss.

Table V.8. Radiated power losses from the TMX central cell and east end cell.

Tab?e VI.1. Calculated central cell axial magnetic conf inement times $\left(\tau_{0}\right)$ and enhanced

electrostatic conf inement times $\left(\tau_{\phi}\right)$ for the ionization states of oxygen observed in TMX. This assumes $n_{p} / n_{c}=3, T_{p}=T_{c}=75 \mathrm{eV}$, $\mathrm{R}=20, \mathrm{n}_{\mathrm{C}}=5 \times 1 \mathrm{Cm}^{12}, \mathrm{~g}(20)=4$.

Tabie A.1 0scillator strengths and average Gaunt factors used to calculate the excitation rates.

Table C.1. QT values vs anode for LLNL-SIDS.

Table C.2. Strip current, yoltage, and gain per MCP of LLNL-SIDS for various voltages from high voltage supply.

Table C.3. QT values vs anode for JHU-SIOS. 


\section{INTRODUCTION}

Until recently, impurities in open-ended magnetically-confined fusion plasmas were not studied extensively. Early mirror devices were plagued with instabilities which resulted in very poor energy confinement [1]. However, with improved control over these instabilities and increased ion temperatures achieved on 2XIIB, the issue of impurities has become more important. The large positive ambipolar potentials Jeveloped in mirror machines (due to the high mobility of the electrons) crupled with the rapid scattering rates of highly-charged impurities into the loss regions of velocity space indicate that impurities should be poorly confined. Low impurity concentrations were, in fact, observed on 2XIIB, except for high-energy neutral-beam-injected oxygen which was well confined. Tandem mirror devices, however, have the first open-ended configuration in which the accumulation of impurities might be possible. In particular, TMX consists of two 2XIIB-type minimum-B mirror end cells connected by a solenoidal (central cell) region of lower magnetic field strength. A higher plasma potential is created in the end cells than in the central cell so that positive ions in the central region are confined axially by a combination of mirror confinement and electrostatic confinement. A significant accumuation of impurities in the large central cell volume would result in a major fraction of the total input power to be lost through radiation. This report presents the first study of impurities performed on a neutral-beam-heated tandem mirror device.

Emphasis was placed upon studying the impurities in the central cell once the end cell impurity concentrations were determined to be similar to those on 2XIIB. The major impurities identified in TMX were carbon, nitrogen, oxygen, and titanium; the concentrations of each in the central cell were less than one-half percent which is much lower than expected (Section V.3). This resulted in only a small fraction of the input power being lost through radiation (Section V.4). The question to address was no longer how to control the impurities, but rather why the impurity concentrations were so low in the central cel1. Two possible answers are either a low source of impurities into the central cell piasma or a low impurity confinement time. Quantitative information regarding impurity sources was not obtained although the condition of the central cell vacuum chamber walls was observed to affect the impurity concentrations (Section V.5.2). The end cell plasmas were not expected to provide a major source of impurities to the central cell; high-energy 
impurities should be well confined within the end cells and low-energy impurities should be preferentially ejected to the end walls of the machine. Experimental evidence of impurity confinement times, however, indicates that the total central cell confinement time for highly-ionized oxygen was on the order of or less than the deuterium confinement time even though calculations predict that the axial confinement time of highly-ionized species should be much longer than the plasma lifetime (Section VI.2). This suggests that radial losses of impurities were important in the central cell plasma. This conclusion is supported by the results of a series of impurity injection experiments performed on TMX. The central cell plasma was observed to resist a radial inflix of the injected impurities (Section VII.3) which is consistent with a radially outward driving force. Furthermore, impurities injected before the plasma build-up were observed to actually leave the central cell plasma (Section VII.4) although none of the impurities were observed in either end cell. The conclusion that outward radial transport results in a short impurity confinement time provides an explanation for the low impurity concentrations in the central cell of TMX.

This report is divided into seven chapters (the first of which is the Introduction) and three appendices. Chapter II contains a brief description of TMX which includes a discussion of tandem mirror physics and the plasma parameters obtained with TMX. The major results of TMX are presented and indicate the success of this tandem mirror experiment. The final section describes TMX-Upgrade which is currently under construction and will test some recent physics concepts which should improve the overali confinement times and efficiency of the tandem mirror configuration.

Chapter III presents the formalism of plasma spectroscopy which was used to analyze the data obtained during this study. Expressions for impurity densities and radiated power losses are obtained along with correction factors required due to the significant Doppler broadening of the impurity emissions from the end cells.

Chapter IV gives a brief description of the three time-resolving monochromators used on TMX during this study. One of the instruments viewed a single chord of plasma at a time while the other two had spatial-imaging capabilities. An outline of the various locations of each instrument on TMX is also given along with a discussion oi the quality of the data taken by each. 
Chapter $V$ is the first chapter on results and presenis the study of the intrinsic impurities found in TMX. A general description of the characteristics of the impurity emissions is given and the impurity concentrations and radiated power losses are determined. Sources of impurities in both the central cell and end cells are discussed.

Chapter VI examines the confinement times of impurities in the central cell of TMX. An expression is developed which predicts the axial confinement times of multiply charged ions. This is compared with experimentally determined values of total confinement time. Aiso presented are the computed effects of finite confinement times on ionization state population ratios.

Chapter VII describes the impurity injection experiments which were performed on the central cell of TMX. The experimental set-up and procedure are explained and the results obtained from two different series of experiments injecting neon and oxygen are presented.

Appendix A contains an extensive set of graphs of ionization, recombination and excitation rate coefficients used in this study. Appendix $B$ presents a flowchart illustrating the software written to take data during the experiments. Finally, Appendix $C$ gives a detailed description of the two spatial-imaging instruments used on TMX and outlines the absolute photometric calibrations performed at Johns Hopkins University. 


\section{THE TANDEM MIRROR EXPERIMENT (TMX)}

\section{II.1. INTRODUCTION}

Since TMX is the first neutral beam heated tandern mirror device, a separate section is devoted here to explain the concepts behind it. Several journal articles have [2-4], or soon will be, published describing various aspects of TMX, so only a brief overview is given here. The first section discusses the physics and enhanced confinement times of TMX. The second section describes the plasma and machine parameters which were designed into this experiment. The third section reviews the major results from TMX and compares them with present theories. Finally, the last section describes the TMX Upgrade which is currently under construction and the improved performance which is expected from it.

\section{I1.2. ENHANCED CONF INEMENT TIMES IN TMX}

One methoc of confining a quasineutral collection of charged particles is by controlling the motion of the ions and electrons with a magnetic field. In particular, a simple magnetic mirror [Fig. II.l(a)] confines a plasma not only in directions perpendicular to the field but confines a large class of particles parallel to the field. However, particles which scatter so that their resulting velocities have a sufficieritly large component parallel to the confining magnetic field will not be reflected by the increasing field strength. This means that there is a class of particles which are not confined by open-ended mirror configurations, i.e., there is a loss region in velocity space. The size of this "loss cone" can be calculated by conserving magnetic moments along a field line and depends upon the mirror ratio of the confining magnetic field:

$$
\sin ^{2} \theta_{0}=\frac{1}{R}=\frac{B_{0}}{B_{m}}=\frac{v_{\perp}^{2}}{v_{0}^{2}}
$$

where $\theta_{0}$ is the pitch angle of the charged particle with respect to the magnetic field in the region of minimum field $B_{0}, B_{m}$ is the maximum magnetic field, $R$ is the mirror ratio $\left(B_{m} / B_{0}\right), v_{\perp}$ is the particle velocity 

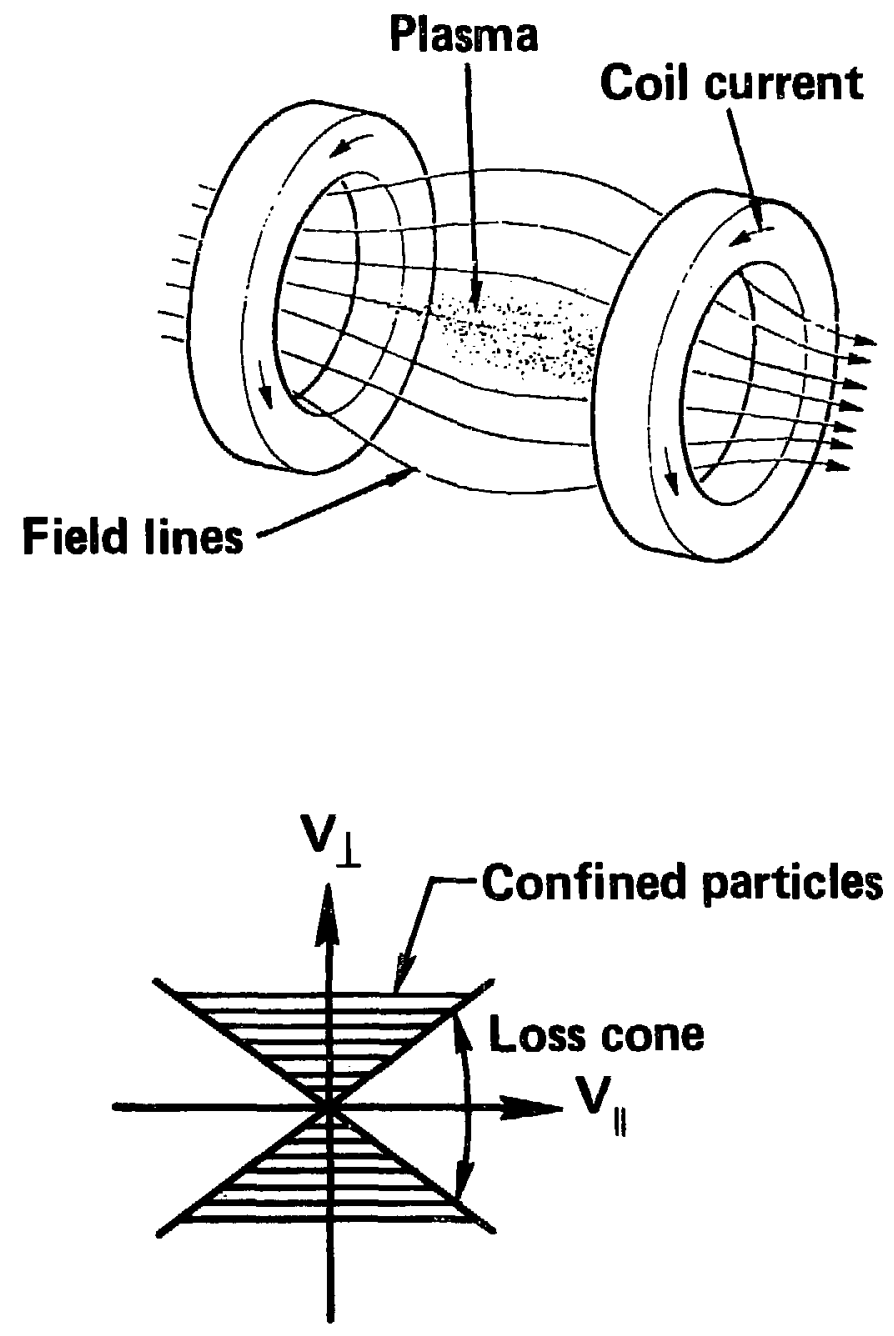

Fig. II.1. (a) Simple magnetic mirror showing increased field strength of each and which tends to reflect charged particles. (b) Loss region in velocity space of an open-ended mirror device. 
perpendicular to the magnetic field and $v_{0}$ is the total velocity of the particle $\left(v_{0}^{2}=v_{1}^{2}+v_{11}^{2}\right)$ [see Fig. II.1(b)].

One consequence of the presence of this loss cone in velocity space is that the velocity distributions are no longer Maxwellian which results in a deviation from thermodynamic equilibrium. This anisotropy of the velocity distribution can drive an instability known as the drift cyclotron loss cone (DCLC) instability. DCLC can severely limit the particle confinement time of an open-ended mirror confined plasma. One method used to minimize this instability is to allow a warm plasma to stream through the mirror parallel to the magnetic field. This has the effect of filling in the loss cone in velocity space, i.e., making the velocity distribution more isotropic and Maxwellian, and bringing the plasma closer to thermodynamic equilibrium. Such a technique was used to stabilize the $2 X I I B$ device by using an external source of warm plasma. On TMX, the plasma flowing out the ends of the central cell was used to stabilize each end cell plasma.

Magnetically confined plasmas develop a large positive potential--known as the ambipolar potential--by virtue of the mass difference between ions and electrons. Electrons, because they are less massive, are much more mobile and collisional than ions and tend to leave the plasma more readily. This results in a separation of charge and establishes a positive electrostatic potential which can be several times the corresponding energy of the electrons. This potential has the effect of equalizing the rate at which the ions and electrons are lost. While the electrons are confined by this ambipolar potential, the ion loss rate is enhanced by about a factor of 2 [5].

TMX uses to advantage the existence of this positive ambipolar potential. The magnetic field, electron density and plasma potential along the length of TMX are sketched in Fig. II.2. From the Boltzmann relation, which assumes quasineutrality along magnetic field lines, a higher potential develops in a region of higher density, so that the potential difference $\phi_{C}$ between the two density regions is

$$
e_{c}=T_{e} \ln \left(\frac{n_{p}}{n_{c}}\right)
$$

for a given electron temperature $T_{e}$, where $n_{p}$ and $n_{c}$ are the electron dens.ties in the end cell regions (plugs) and central region of TMX respectively, and $\mathrm{e}$ is the electron charge. (The electrons are assumed to be mobile enough to have a constant temperature along a given field line over the 


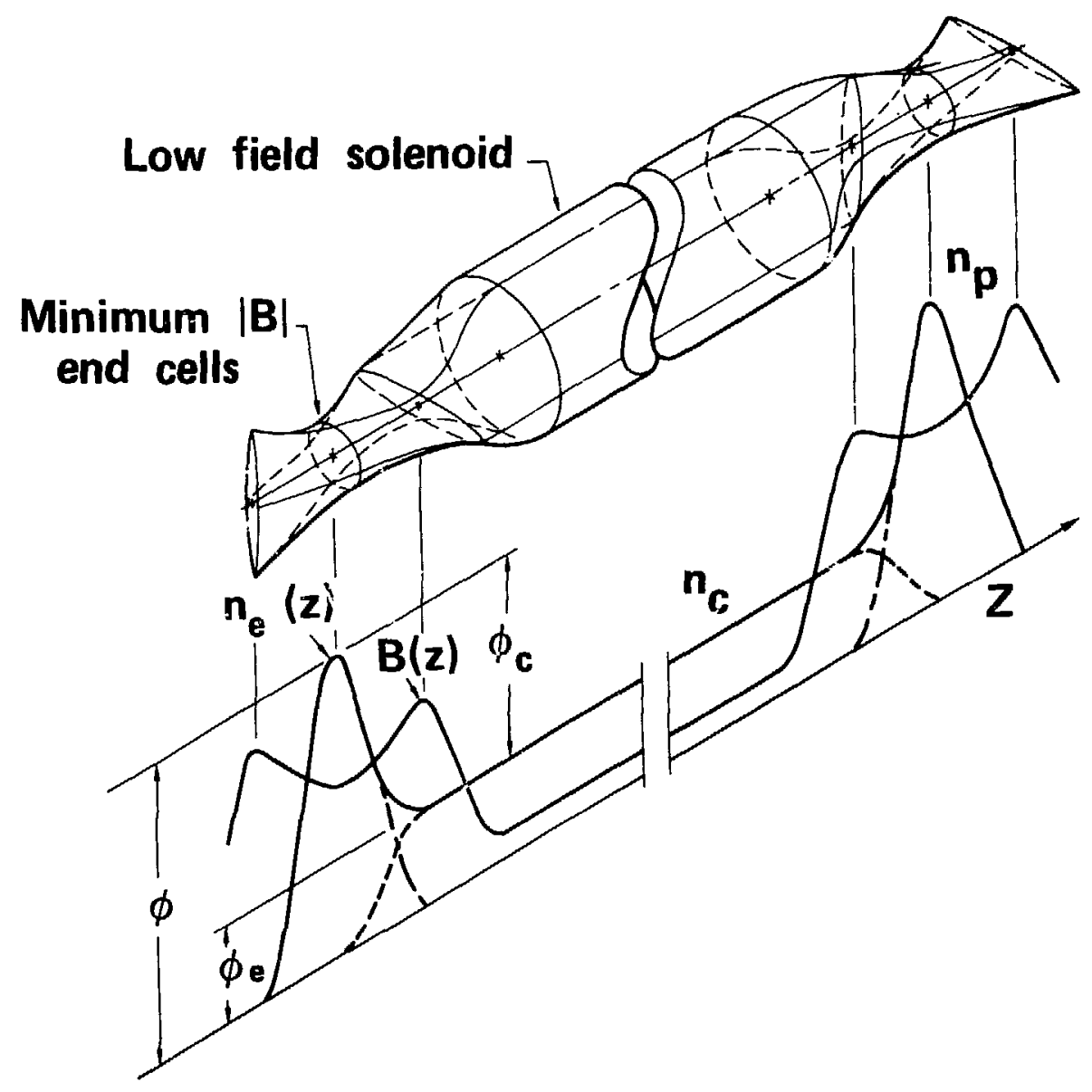

Fig. II.2. Magnetic field, electron density, and plasma potential along the length of TMX. 
length of TMX.) It can be seen from this relation that $n_{p}>n_{c}$ must be satisfied in order that $\phi_{c}>0$. Furthermore, a heating scheme which preferentially heats the electrons is most effective in increasing $\phi_{C}$. (A detailed description of the machine and the method of maintaining density gradients in the plasma will be given in the next section.)

The ions in the central cell of TMX, then, are confined not only by the higher magnetic fields at each end of the machine, but are further confined by the presence of the positive electrostatic potential which tends to repel the ions as they approach the ends of the machine. It has been shown [6] that deuterons $(Z=1)$ in the central region are confined for a time $\tau_{c}$, which is required for them to diffuse upward in energy until they can escape over the potential barriers (for $\mathrm{e}_{\mathrm{c}} \geq 2 \mathrm{~T}_{\mathrm{e}}$ ):

$$
\tau_{c}=\tau_{i j} g(R) \frac{e \phi_{c}}{T_{c}} \exp \left(\frac{e \phi_{c}}{T_{c}}\right)
$$

where $\tau_{i j}$ is the deuteron-deuteron collision time, $T_{c}$ is the central cell ion temperature, and $g(R)=\pi(2 R+1) \ln (4 R+2) / 4 R$ where $R$ is the mirror ratio. Combining the two above equations gives:

$$
\tau_{c}=\tau_{i j} g(R)\left(\frac{T_{e}}{T_{c}}\right) \ln \left(\frac{n_{p}}{n_{c}}\right)\left(\frac{n_{p}}{n_{c}}\right)^{T^{/ / T}} .
$$

Maintaining $T_{e}>T_{c}$ and $n_{p}>n_{c}$ allows an enhanced confinement time of ions in the central region of TMX. The primary objective of TMX was to provide a proof-of-principle of the enhanced confinement of deuterons in the central region of a tandem mirror geometry.

This erihanced confinement time of ions raised concern regarding the presence of impurities in the central cell plasma. The electrostatic confinement times should increase with increasing charge state of an ion, therefore, impurities should be more effectively confined axially than the working gas deuterium. It is well known, especially in tokamak work, that impurities can provide a major loss of power from the plasma through radiation; furthermore, impurities can enhance certain instabilities in plasmas. The original motivation for this research was to determine absolute impurity densities and radiated power losses from the central plasma in TMX. 


\section{II.3. TMX PARAMETERS}

TMX consists of three regicns: two end cells and a central cell. Each end cell is a 2XIIB-type neutral-beam-heated mirror device [7]; that is, each has its own minimum-B field configuration with a $2: 1$ mirror ratio (20 kG:10 kG). The central cell is a $5 \mathrm{~m}$ long solenoidal region of lower magnetic field strength $(0.5-2 \mathrm{kG})$. Ficure II.3 shows the timing sequence for typical TMX ope ation. A warm plasma is injected along field lines to provide a target for the high energy beams of neutral deuterium which are injected perpendicular to the magnetic field (Fig. II.4). A high density plasma can be built up in each end cell as the high energy neutral particles charge exchange with the warm ions to yield warm neutral particles and high energy ions. These high energy ions have most of their energy perpendicular to the magnetic field and are, therefore, well confined within each end cell. The density of the end cell plasmas, then, is controlled by the amount of injected neutral beam current ( $n_{p}$ is typically $1-3 \times 10^{13} \mathrm{~cm}^{-3}$ with 150 equivalent atomic amps of beam current per end cell). The density of the central cell plasma is controllea by adjusting the amount of gas allowed to flow into that region $\left(n_{c}=0.3-1 \times 10^{13} \mathrm{~cm}^{-3}\right)$. There are two methods by which gas is provided to the central cell and each results in different radial plasma profiles. One technique is simply to open a small gas valve at the midplane of the central ce11. This is known as "puffer operation." The other technique is to inject gas near each end of the central region where the plasma shape is very elliptical (the radii are $2 \mathrm{~cm} \times 57 \mathrm{~cm}$ ), the gas should readily penetrate to the innermost flux lines at these locations before ionizing. The gas is injected via a set of collars which surround the plasma with the same ellipticity as the flux bundle. This is known as "gas box operation." Whichever central cell gas fueling method is used, the plasma density in the central region of TMX can be adjusted independently (within limits, of course) of the density in the end cells $\left(n_{p} / n_{c} \sim 3\right.$ for most of TMX operation). This is crucial in maintaining a potential difference between the plugs and central cel1.

In a tandem mirror reactor, the bulk of the fusion reactions will take place in the central region so that the ions there must be sufficiently heated in addition to being well confined. In TMX, the central cell ions are heated in the following manner. Al1 of the energy contained in the TMX plasma is provided by the neutral beams injected into the end cells. The high energy 


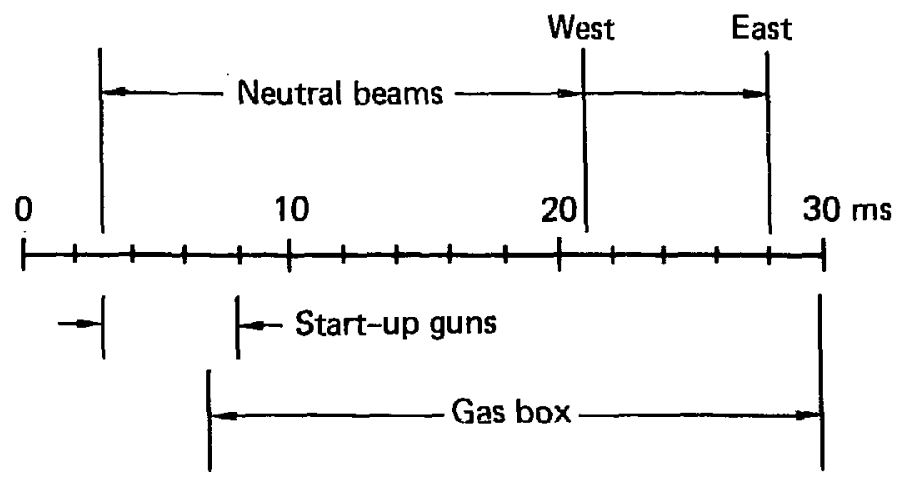

Timing sequence on $\operatorname{TMX}(9 / 26 / 80)$

Fig. II.3. Timing sequence for typical $T M X$ operation. The gas feed could be via either the gas boxes or puffer valves. 


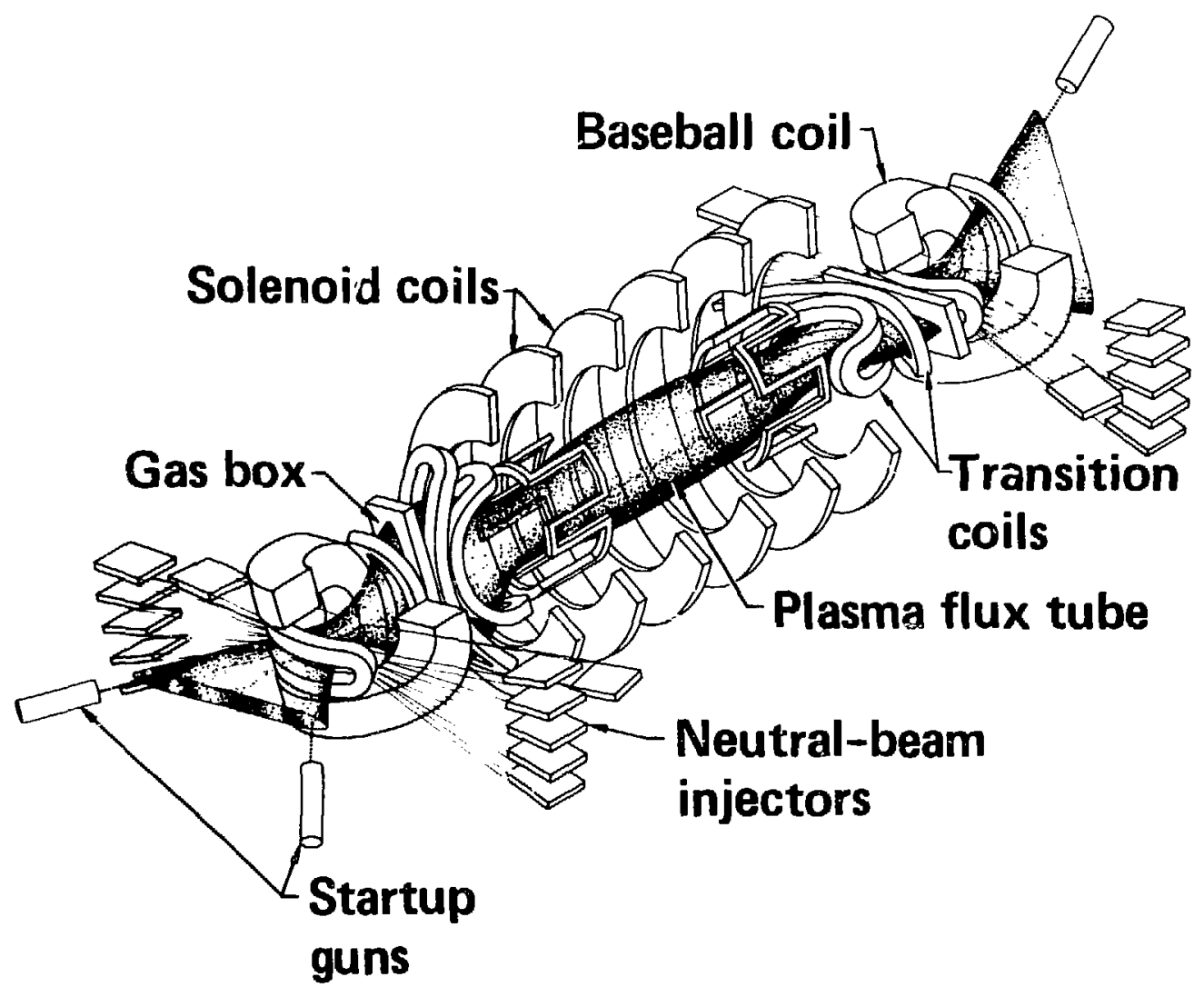

Fig. II.4. Schematic of TMX showing geometry of the magnet system, neutral beams, start-up guns and magnet ic flux bundle. 
ions trapped in the plugs transfer energy to the electrons in the plug by Spitzer drag [8]. The electrons are sufficiently mobile not only to maintain a Maxwellian velocity distribution but also to be able to sample the entire plasma axialiy. Therefore, the electrons are heated in the plugs and then eventually transfer their energy to the ions trapped in the central cell. The energies of the various particles, then, have the following relationship:

$$
\mathrm{W}_{\text {ip }} \gg \mathrm{T}_{\text {ep }} \geq \mathrm{T}_{\text {ec }}>\mathrm{T}_{\text {ic }}
$$

where $\bar{W}_{i p}$ is the average ion energy in the plug (the plug ions have a highly non-Maxwellian velocity distribution, so no temperature is assigned to their energy), $T_{e p}$ and $T_{e c}$ are the plug and central cell electron temperatures and $T_{i c}$ is the central cell ion temperature. Typical values for the particle energies are $W_{i p} \sim 10 \mathrm{keV}, T_{\text {ep }}=\mathrm{T}_{\text {ec }}=50-100 \mathrm{eV}$ and $\mathrm{T}_{i c}=50-100 \mathrm{eV}$. Other plasma parameters of TMX operation are given in Table II.l.

\section{II.4. MAJOR RESULTS FROM TMX}

The experiments on TMX have demonstrated that the basic tandem mirror principles are correct. The main result is that TMX established electrostatic confining potentials which improved the central cell confinement. Up to a factor of 9 enhancement over the case without electrostatic conf inement was observed for deuterium, although factors of 3 to 7 were more typical [3]. This corresponds to a 150-300 volt potential well depth between the end cells and the central cell. Several factors were important in achieving this result. Maintaining higher densities in the end cells than in the central cell was a crucial requirement. Plug densities were generally three to five times higher than central cell densities. Achieving high electron temperatures was also an important factor. Electron temperatures as high as $260 \mathrm{eV}$ were obtained in the east end cell. This is three to four times higher than temperatures obtained with similar beam currents in 2XIIB. Isolating the plasma from the end walls of the vacuum chamber was necessary to achieve high electron temperatures. This isolation was achieved by allowing the warm plasma flowing out the ends of the central cell to stabilize the end cells (as discussed in Section II.2) rather than using an external source of warm plasma as in $2 X I I B$. 
Table II.1. Typical operating parameters for TMX compared with expected values for TMX-Upgrade.

Parameters TMX TMX-U [7]

Central cell

Density, $n_{c}\left(\mathrm{~cm}^{-3}\right)$

Electron temperature, $\mathrm{T}_{\mathrm{ec}}(\mathrm{keV})$

Ion temperature, $T_{\text {ic }}(\mathrm{keV})$

$P$ lasma length, $L_{c}(m)$

$P$ lasma radius, $r_{c}(\mathrm{~cm})$

Magnetic field, $B_{c}(k G)$

Confining potential, $\phi_{C}$ (keV)

Conf inement lifet ime, $\tau_{c}$ (ms)

End Cell

Density, $n_{p}\left(\mathrm{~cm}^{-3}\right)$

Electron temperature, $T_{\text {ep }}(\mathrm{keV})$

Ion energy, $w_{j}$ (keV)

Mirror-to-mirror length, $L_{p}$

$p l$ asma radius, $r_{p}(\mathrm{~cm})$

Central magnetic field, $B_{p}$

Mirror ratio (axial), $R$

(m)

(kG)

Barrier

Density, $n_{b}\left(\mathrm{~cm}^{-3}\right)$
$5 \times 10^{12} \quad 2 \times 10^{13}$

0.1

0.6

0.1

0.9

5.5

8.0

25

1

25

0.3

2.2

3

17
0.1

10

0.9

10

10

2

$-\quad 5 \times 10^{12}$ 
With increased axial confinement from the plug potentials, the question of radial confinement in the central cell became an important issue, sspecially since there are regions between the plugs and the central cell where the curvature of the magnetic field lines might promote radial transport of the ions [2]. Experimental results indicate, however, that the radial confinement time of the deuterons exceeds the axial confinement time near the plasma axis, although radial transport processes become more important near the plasma edge. Most of the net input power from the neutral beams is deposited at the end walls rather than at radial positions.

Not all observations on TMX, however, were expected from the theory in the original proposal. Equation (II.2) for the increased electrostatic potential in the plugs assumes that the electron temperature is equal along field lines throughout TMX. It was often found, however, that the end cell electron temperature exceeded that in the central cell by about a factor of 2 . According to theory [9], some of the TMX data suggests that there is a region between the end cells and central cell where the density drops to about half the central cell density. This region of low density is referred to as a thermal barrier since the plug and central cell electrons would then be isolated from each other and would not necessarily have the same temperature. This thermal barrier is the basis for the design of the TMX Upgrade and will be discussed in the last section of this chapter. Several atiempts were made to observe the thermal barrier region with microwave interferometers but none were successful.

Another originally unexpected observation was made regarding the central cell ion temperatures. Measurements made with diamagnetic loops of total central cell plasma energy ( $k T_{\text {ion }}+k T_{\text {electron }}$ ) suggested that the central cell ion temperatures were often higher than the electron temperatures. This was eventually supported by Doppler broadening measurements which indicated that central cell ion temperatures might be as high as 100-200 eV [10]. Theory now indicates that radio-frequency (RF) fluctuations generated in the end cells couple to the central cell ions and heat them [11]. Increased RF fluctuations were also associated with decreased ion confinement in the central cell.

One final remark will be made here for completeness. The results of this study indicate that the impurity concentrations were considerabiy lower $(\leqslant 0.5 \%)$ than expected in the central cell of TMX. Experimental evidence suggests that enhanced outward radial transport of impurities reduced the 
impurity confinement times in the central cell. This will be discussed in detail in Chapters V-VII.

In summary, TMX established increased central cell confinement of deuterium ions in accordance with fundamental tandem mirror theory. This result has provided support for scaling laws for tandem mirror confinement times and the construction of TMX Upgrade [12] with thermal barriers and the much larger MFTF-B [13] which will extend TMK results to thermonuc lear parameters.

\section{II.5. TMX UPGRADE}

Construction is clirrently in progress of a modified tandem mirror experiment--called the TMX Upgrade [12]--which uses to advantage the concept of the thermal barrier mentioned in the previous section. Recall that in Eq. (II.2) the potential is proportional to the electron temperature times the natural logarithm of the density. Suppressing the density in a region between each end cell and the central cell would create a potential dip (see Fig. II.5) between the central cell and piugs that would isolate the electrons in those regions. This means that the central cell electron temperature need not be equal to that in the plugs. In particular, the effort on TMX Upgrade will be to greatly increase the electron temperature in the plugs by auxiliarly heating [alectron cyclotron resonance heating (ECRH)]. This means tnat a large positive confining potential can be established in each end cell at a much lower end cell density. This is the main advantage of the thermal barrier. In fact, the plug density could now be considerably lower than the central cell density rather than the reverse case required on TMX. Not only does this relax the technological requirements for construction by greatly reducing magnetic field strengths and neutral beam energies in the plugs, but also significantly raises the overall efficiency of a tandem mirror reactor.

The details of maintaining a density profile which includes a thermal barrier are lengthy so will not be discussed here [12]. However, the relationship giving the potential difference created between the plugs and central cell in TMX by assuming a single electron temperature [Eq. (II.2)] now becomes more general and is given by:

$$
\phi_{c}=T_{e p} \ln \left(\frac{n_{p}}{n_{b}}\right)\left(\frac{T_{e c}}{T_{e p}}\right)^{1 / 2}-T_{e c} \ln \left(\frac{n_{c}}{n_{b}}\right)
$$



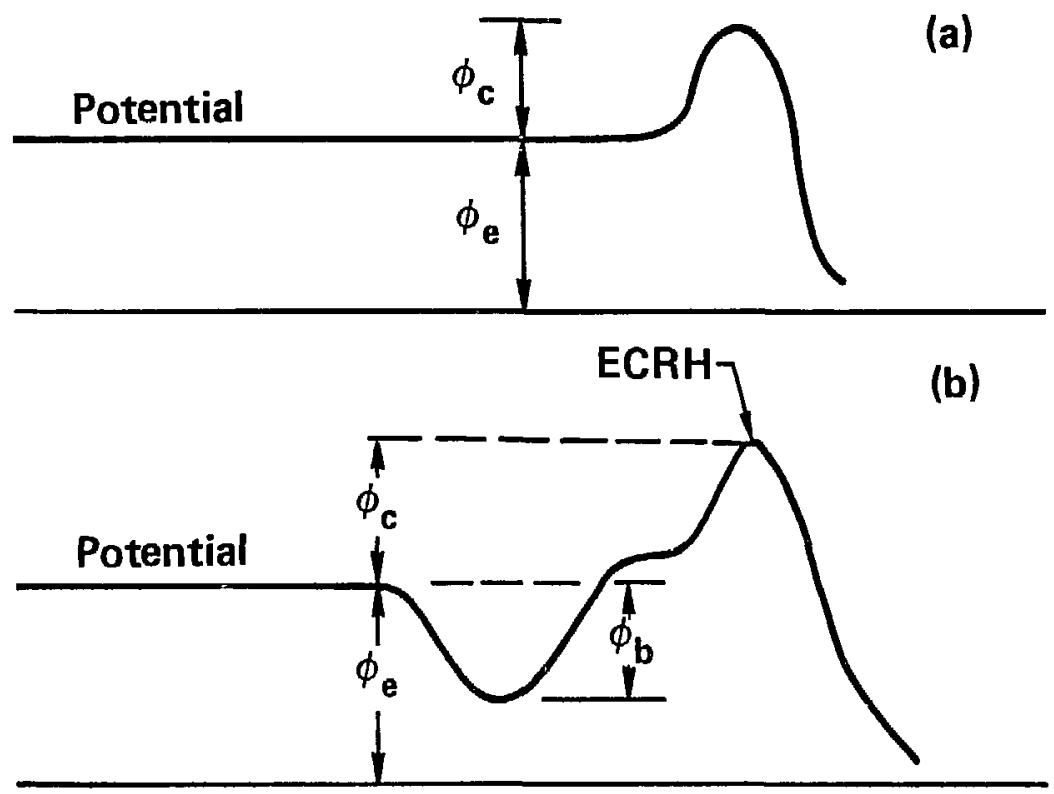

Fig. II.5. Comparison of plasma-potential profiles: (a) basic tandem potential profile (TMX), and (b) potential profile with thermal barriers (TMX Upgrade). $\phi_{c}=$ conf inement potential, $\phi_{e}=$ central cell potential, and $\phi_{b}$ = potential depression at the barriers. 


$$
-28-
$$

where $T_{e p}, T_{e c}$ are the electron temperatures in the plugs and central cell; $n_{p}, n_{b}, n_{c}$ are the densities in the plugs, thermal barriers and central cell. Obtaining maximum $\phi_{c}$ requires $T_{e p} \gg T_{e c}$ and $n_{p} / n_{b} \gg 1$. Note that $n_{p}>n_{c}$ is no longer required as in the case of TMX. Table I.1 lists the expected parameters on TMX-Upgrade compared with TMX. 


\section{EUV PLASMA SPECTROSCOPY ON TMX}

\section{III.1. INTRODUCTION}

The use of spectroscopy to observe and analyze high temperature plasmas goes beyond the applications to laboratory plasmas. Spectroscopy has been essentially the only means available for species identification in stars and other astrophysical objects. Similarly, spectroscopy is very useful for identifying impurities in magnetically confined fusion plasmas. The use of spectrometers which are calibrited for intensity measurements allows the determination of actual impurity densities, assuming that other parameters such as electron density and temperature are known. Radiated power losses may also be determined by these techniques. This chapter discusses the formalism which was applied to the data to arrive at the results presented in this report. Section 2 develops an expression for the impurity ion density starting from the observed brightness. Section 3 studies two different ion density radial profile models, and Section 4 briefly describes the calculations for radiated power losses from the plasma. Finally, Section 5 discusses corrections to observed brightnesses of Doppler broadened emission Tines.

\section{III.2. BRIGHTNESSES AND ION CENSITIES}

The quantity measured by spectroscopic instruments is the brightness, which is proportional to the number of photons per second emitted by the plasma at a given wavelength. Assuming that the plasma is optically thin, the spectrometer receives photons from the entire line of sight of plasma integrated over the area of plasma subtended by the entrance slit. Taking into account the efficiency of the optics and detector, the brightness may be expressed as

$$
B=\frac{N}{(Q T) \Omega_{p} A_{s}}\left(p h-s^{-1}-\mathrm{cm}^{-2}-s r^{-1}\right)
$$

where $N$ is the observed counting rate, QT is the quantum efficiency of the detector times the transmission of all optical components in the instrument and beamline, $\Omega_{p}$ is the solid angle of plasma subtended by the entrance slit, and $A_{s}$ is the area of the entrance slit. 
The number of photons emitted by the plasma is determined by the rates at which the various processes occur within the plasma. In TMX plasmas, electron impact excitation is the dominant excitation process. Radiative de-excitation is a much faster process, so that as soon as an ion reaches an excited state, it immediately radiates a photon and returns to the ground state. This means that essentially all ions exist in the ground state or low-level metastable states.

Since electron impact excitation is the process which determines the rate at which photons are emitted, the volume emission rate $E$ is given by

$$
E_{i}=n_{i} n_{e}^{\langle\sigma v\rangle_{i}^{e x}}
$$

Where $n_{j}$ is the density of the given ionization state, $n_{e}$ is the electron density, and $Q_{i} \equiv\langle\sigma\rangle_{i}^{e x}$ is the rate coefficient for electron impact excitation.

The brightness is obtained by integrating the volume emission rate along the line-of-sight chord of plasma viewed by the instrumeni:

$$
B_{i}=\frac{1}{4 \pi} \int E_{i} d l
$$

In order to obtain the volume emission rate from the brightness, an Abel transformation must be performed on the above equation. The Abel transform and an equivalent matrix inversion technique assume cylindrical symetry and are discussed thoroughly in other references $[14,15]$.

Once $E_{j}(r)$ is known, the impurity density is simply

$$
n_{i}(r)=\frac{E_{j}(r)}{n_{e}(r) Q_{j}(r)} .
$$

Radial profiles of the electron density and excitation rate (discussed in Appendix A) also must be known in order to calculate the ion density radial profile.

\section{III.3. ION DENSITY RADIAL PROFILE MODELS AND CONCENTRATIONS}

The inversion of a brightness radial profile accentuates noise in the data and does not provide an analytic form for the ion density (unless some fit is made to the resulting points) to use in further calculations. Therefore, it is often useful to describe the ion density in Eq. (111.2) with 
a suitable model. In Chapter $V$, two models are shown to describe the ion density radial profiles: a constant density mode 1 and a constant concentration mode1. In the end cells of TMX, the singly- and doubly-ionized states have nearly constant density profiles while the higher ionization states are described with the constant concentration model. These are the same models used to describe the radial ion profiles on 2XIIB [16]. In the central cell, all ionization states of all impurities are described as having constant densities with radius.

The constant density model assumes that the ion density is a constant function of radius. Furthermore, Appendix $A$ indicates that the excitation rate coefficient is a slowly varying function for most temperatures observed on TMX, so that $Q_{i}$ is assumed constant also in this model. Therefore, Eq. (III.3) becomes

$$
n_{i}=\frac{4 \pi B_{i}}{Q_{i} \int n_{e} d l}
$$

This is the expression for the ion density in the constant density model. Note that $B_{i}$ and $\int n_{e}$ d $l$ have the same radial profile according to this model.

The second model used in Chapter $V$ assumes that the ion density and electron density have the same radial profiles, i.e., a constant concentration. Equation (III.3) now becomes $\left(Q_{j}\right.$ is assurned constant in this model also)

$$
c_{i}=\frac{n_{i}}{n_{e}}=\frac{4 \pi B_{i}}{Q_{i} \int\left[n_{e}(r)\right]^{2} d l}
$$

In this mode $1, c_{j}$ is a constant function of radius.

\section{III.4. RADIATED POWEP LOSSES}

The power radiated from the plasma may be calculated directly from the volume emission rates. At a given wavelength $\lambda_{j}$, the radiated power $P_{i}$ is given by

$$
P_{i}=\frac{h c}{\lambda_{i}} \int E_{i} d^{\prime \prime}
$$


where the volume emission rate $E_{j}$ is integrated over the entire plasma voiume. The excitation rates are assumed constant as in the density calculations.

In the central cell, all impurity ions are assumed to have constant density as before; the electron density is assumed Gaussian radially and constant along the effective length of the central cell $\left(L_{\text {eff }}=314 \mathrm{~cm}\right)$. In the end cells, the impurity ions are assigned the same distributions used for the density calculations; the electron density is assumed Gaussian both radially and axially in accordance with the plasma line density data. The 1/e radius varies from 8 to $10 \mathrm{~cm}$, while the length is generally 12-14 cm.

Equation (III.7) gives the radiated power for only a single transition, while the desired quantity is the total power radiated from all transitions for each impurity species. Since it is not practical to observe all transitions with a monochromator, it is possible to estimate the contributions of the unobserved transitions by measuring the brightness of a transition to each ground state and low-lying metastable state and then scaling the brightnesses of the unobserved transitions to these states according to their respective excitation rates. The assumptions and formalism for this procedure are discussed in detail elsewhere $[16,17]$ so will not be presented here.

\section{III.5. DOPPLER CORRECTIONS}

The ion energies in the end cells of TMX are often in excess of $10 \mathrm{keV}$, so that the emission lines are substantially Doppler broadened. The measured widths of these lines allow an estimate of the ion energy. Since these high-energy ions are mirror confined and have most of their velocity perpendicular to the magnetic field, the resulting ion velocity distribution is highly non-Maxwellian. Therefore, the appropriate expression for the ion energy is that of its kinetic energy [16]:

$$
\begin{aligned}
& E=\frac{1}{2} A\left(m_{p} c^{2}\right)\left(\frac{\Delta \lambda}{\lambda}\right)^{2} \\
& E(k e V)=4.69 \times 10^{5} A\left(\frac{\Delta \lambda}{\lambda}\right)^{2}
\end{aligned}
$$

Where $A$ is the atomic mass, $m_{p}$ is the proton mass, $c$ is the speed of light, $\lambda$ is the wavelength of the emission line observed, and $\Delta \lambda$ is the half-width at half-maximum (HWHM) of the spectral profile. Equation (III.8) is used to determine the ion energies presented in Chapter $V$. 
The brightnesses of the emissions from the plug are often so high that the size of the spectrometer slits has to be reduced until the widths correspond to a spectral resolution which is iess than the spectral widths of the emission lines. Therefore, the observed brightnesses are less than the actual brightnesses of those lines. The following section derives the correction factor required in the case of a Doppler-broadened singlet while Section III.6.2 addresses the more general case of a Doppler-broadened and unresolvable multiplet.

\section{III.5.1 Doppler-Broadened Singlet}

The total brightness ( $B_{\text {tot }}$ ) contained in a Gaussian emission line is given by

$$
B_{\text {tot }}=\int_{-\infty}^{\infty} B_{0} \exp \left\{-\left[\left(\lambda-\lambda_{0}\right) / w\right]^{2}\right\} d\left(\lambda-\lambda_{0}\right)
$$

where $\mathrm{B}_{0}$ is the peak brightness, $w$ is the e-folding half-width and the integral is performed over all wavelengths $(\lambda)$ relative to the peak wavelength $\left(\lambda_{0}\right)$ for $\lambda_{0} \gg w$. Performing the integral yields

$$
B_{\text {tot }}=B_{0} \sqrt{\pi} W \text {. }
$$

The measured brightness ( $B_{\text {meas }}$ ) is the emission profile in Eq. (III.9) modulated by the response of the spectrometer which is actually triangular if the entrance and exit slit widths are equal but may be approximated by a Gaussian with only small errors. Therefore, the brightness measured at a given wavelength $\left(\lambda_{\mathrm{I}}\right)$ is

$$
B_{\text {meas }}\left(\lambda_{I}\right)=\int_{-\infty}^{\infty} B_{0} \exp \left\{-\left[\left(\lambda-\lambda_{0}\right) / w\right]^{2}\right\} \exp \left\{-\left[\left(\lambda-\lambda_{I}\right) / w_{I}\right]^{2}\right\} d\left(\lambda-\lambda_{0}\right)
$$

where $w_{I}$ is the e-folding half width of the instrumental spectral resolution. If the instrumental response is centered at $\lambda_{0}$, then $\lambda_{I}=\lambda_{0}$ and the integral yields

$$
B_{\text {meas }}^{0}=B_{0} \sqrt{\pi} w_{0}
$$


where $B_{\text {meas }}^{0}$ is the maximum brightness observed and

$$
\frac{1}{w_{0}^{2}}=\frac{1}{w^{2}}+\frac{1}{w_{I}^{2}}
$$

Eliminating $B_{0}$ from Eqs. (III.10) and (III.12) and substituting Eq. (III.13) for $w_{0}$ gives

$$
B_{\text {tot }}=\left[\left(1+\frac{w^{2}}{w_{I}^{2}}\right)^{1 / 2}\right]_{\text {meas }} .
$$

Note that in the case of very little Doppler broadening compared to the instrumental resolution, as in the central cell, $w \ll w_{I}$ and $B_{\text {tot }}=B_{\text {meas }}^{0}$ so that no correction factor is needed. Going one step further and using the fact that the measured width $\left(w_{m}\right)$ of the emission line is given by (adding Gaussians in quadratures)

$$
w_{m}^{2}=w^{2}+w_{I}^{2}
$$

yields

$$
B_{\text {tot }}=\frac{W_{m}}{W_{I}} B_{\text {meas }}^{0}
$$

The total brightness is merely the ratio of the measured line width to the instrumental width times the maximum observed brightness.

\section{III.5.2 Doppler-Broadened Multiplet}

The situation becomes more general in the case of a multiplet in which the individual emission lines are sufficiently Doppler broadened that they cannot be resolved. Equations (III.9) and (III.11) become

$$
B_{\text {tot }}=\int_{-\infty}^{\infty} \sum_{j} B_{j} \exp \left[-\left[\left(\lambda-\lambda_{j}\right) / w\right]^{2}\right] d\left(\lambda-\lambda_{j}\right)
$$

and

$B_{\text {meas }}\left(\lambda_{I}\right)=\int_{-\infty}^{\infty} \sum_{j} B_{j} \exp \left\{-\left[\left(\lambda-\lambda_{j}\right) / w\right]^{2}\right\} \exp \left\{-\left[\left(\lambda-\lambda_{I}\right) / w_{I}\right]^{2}\right\} d\left(\lambda-\lambda_{j}\right)$ 
where each line in the multiplet is centered at $\lambda_{j}$ and each line has a width $w$. A routine was written using the above equations which calculates the width of each line of a Doppler-broadened multiplet if the measured total width and oscillator strength of each line are given. This routine is used to calculate the ion energies of species in which an unresolved multiplet was scanned in wavelength. 


\section{IV.1. INTRODUCTION}

Three absolutely-calibrated $0.4 \mathrm{~m}$ normal incidence monochromators were used on TMX during the course of this study. The first instrument is the original $0.4 \mathrm{~m}$ built at Johns Hopkins University. It is equipped with a standard photomultiplier tube detector which viewed a single chord of plasma per event and is referred to as the "single channel instrument." The second $0.4 \mathrm{~m}$ used on TMX is equipped with a spatial imaging detector system (SIDS). It was purchased by LLNL and was designed and build specifically for this experiment and is referred to as the "LLNL-SIDS." The third instrument brought to Livermore was originally used by S. L. Allen on Alcator A at M.I.T. and has as its detector the original SIDS designed and built by R. K. Richards, H. W. Moos, and S. L. Allen at Johns Hopkins University. It is called the "JHU-SIDS" in this report.

Using three instruments necessarily means that preparation, maintenance, and calibration composed a large fraction of the total effort that went into this project. This chapter, therefore, is devoted co a general description of the single channel instrument and the two spatial-imaging instruments. Details of the construction and calibration results for the LLNL-SIDS and the JHU-SIDS are given in Appendix $C$. The location of each instrument on TMX is also described in this chapter along with a discussion of the quality of data obtained by each.

\section{IV.2. THE SINGLE CHANNEL INSTRUMENT}

This $0.4 \mathrm{~m}$ normal incidence monochromator has a photomultiplier detecior so it has no spatial imaging properties. It has been the traditional workhorse of the plasma spectroscopy group at $\mathrm{JHU}$, and was the first instrument taken to LLNL for this project. It was originally calibrated on the Synchrotron Ultraviolet Radiation Facility (SURF II) electron storage ring at the National Bureau of Standards in Gaithersburg, Maryland, and since then on the Extreme Ultra-Violet Calibration Equipment at JHU (see Appendix C). This instrument has been used on Versator $I$ and Alcator $A$ at M.I.T. and also on 2XIIB at LLNL. During that period of three years, its absolute photometric 
calibration had not degraded more than $50 \%$. This instrument has been described in detail elsewhere [18], so only a brief description of it is given here.

The $0.4 \mathrm{~m}$ monochromator itself is similar to those used for the LLNL-SIDS and JHU-SIDS (Appendix C) with the exception of the grating. Since this instrument is not designed for spatial imaging, there is no need for an enlarged field of view. Therefore, the grating is smaller than in the other two instruments; it measures $20 \mathrm{~mm} \times 20 \mathrm{~mm}$. This grating was made by Bausch and Lomb and was coated with platinum. It was ruled with 24001 ines/mm so that its linear dispersion is the same as that of the LLNL-SIDS (100 $\mu$ slits $=1 \AA$ resolution) but the blaze wavelength is $300 \AA$ as on the JHU-SIDS.

The detector is a windowless EMR $510 \mathrm{~W}$ photomultiplier tube (PMT) which has had its first dynode coated with approximately $1000 \AA$ of $\mathrm{CU}$ I to enhance its quantum efficiency above $1200 \AA[19]$. The analog signal from the PMT is converted from a current to a voltage by a fast electrometer and was then sent into the main TMX data computer (HP-1000) where the signal was digitized and stored on magnetic tape along with all data taken by the other diagnostics on TMX. The PMT and the electronics package (high voltage supply and curre: amplifier) mounted directly behind it are protected from $x$-rays by lead shielding and are surrounded by magnetic shielding which has been tested to 500 gauss longitudinal and 1000 gauss transverse magnetic fields. A schematic drawing of the entire instrument is given in Fig. IV.l.

A graph of the photometric response vs wavelength is given in Fig. IV.2. The pre-TMX calibration (1/79) indicated a factor of 2 drop in sensitivity at $1216 \AA$ from the value obtained before its use on 2XIIB $(8 / 78)$. The post-TMX calibration (6/81) showed no change in QTG during the use of this instrument on TMX.

\section{IV.3. SPATIAL-IMAGING MONOCHROMATORS}

These instruments are the combination of a $0.4 \mathrm{~m}$ normal incidence monochromator and a spatial-imaging detector system (SIDS) [20]. Details of these instruments are given in Appendix $C$. This section contains a brief description of the spatial-imaging system.

The optics in this system are both simple and yet quite free from aberrations. Figure IV.3 sketches a ray diagram of the essential imaging 


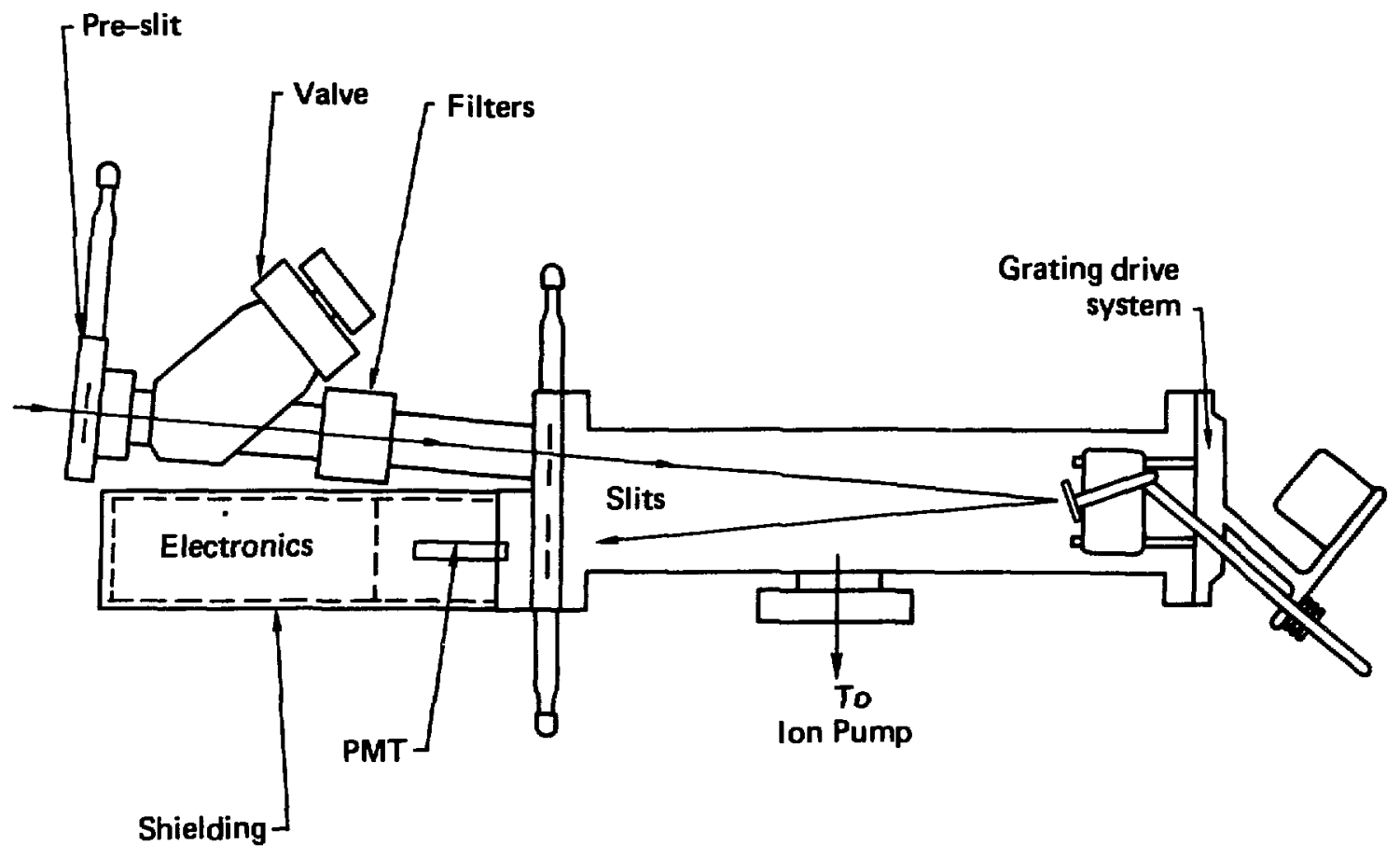

Fig. IV.1. Schematic diagram of the single channel instrument. 


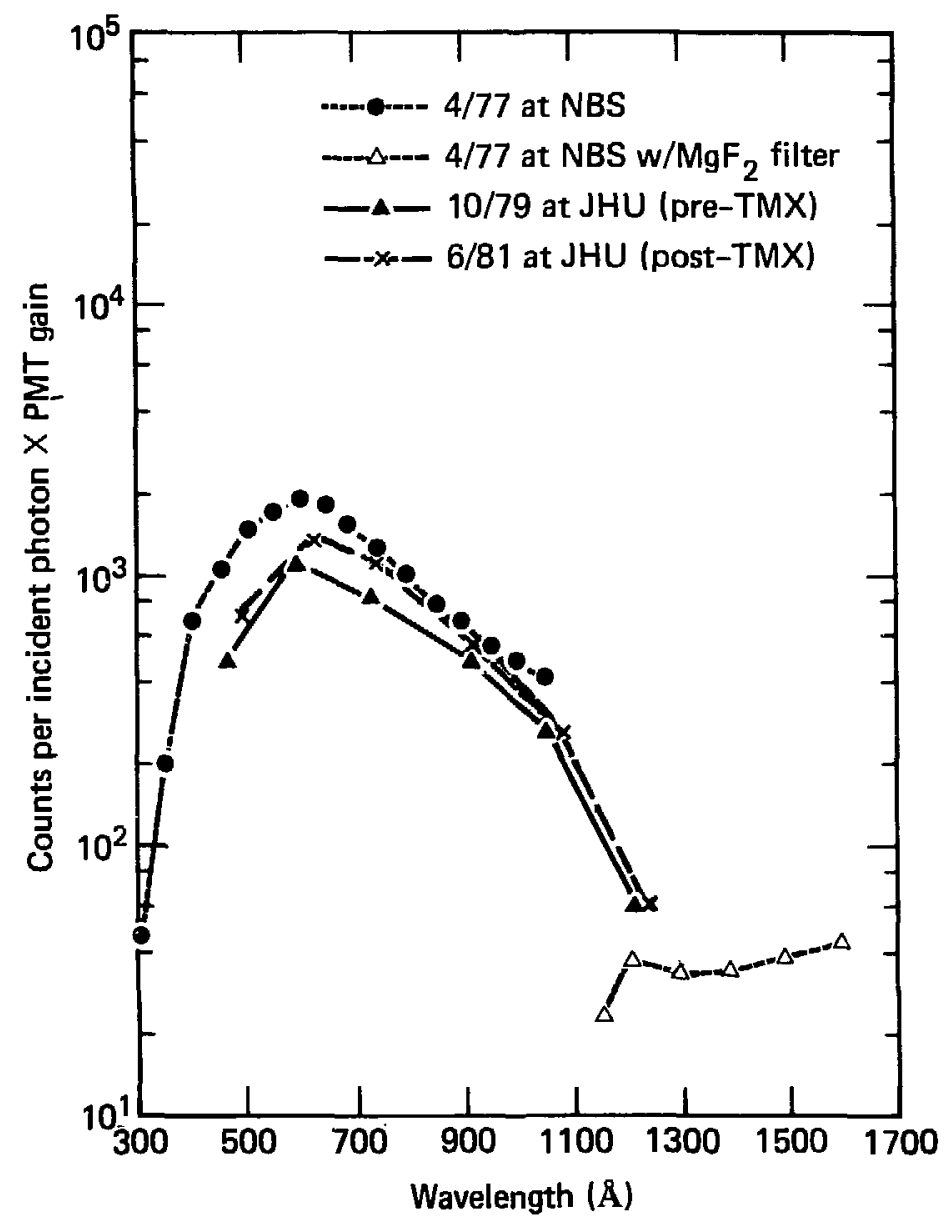

Fig. IV.2. Photometric calibration vs wavelength for the single channel instrument. 


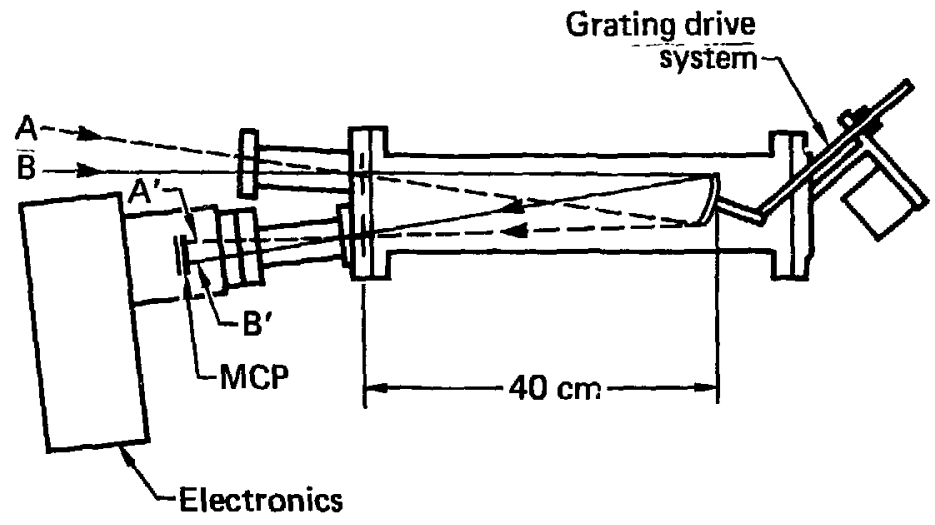

Fig. IV.3. Ray diagram of spatial imaging optics in the LLNL-SIDS instrument. 
components. The plasma (or other extended source of photons) is located to the right of the drawing. Different points $(A$ and $B$ ) in the plasma illuminate different locatic.ss on the grating which then illuminate different anodes ( $A$ ' and $\mathrm{B}^{\prime}$ ) of the detector. Therefore, each anode of the detector views a different spatial region of the plasma. Note that the long dimensions of the entrance and exit slits, the grooves in the grating and the long (or non-imaging) dimensions of the detector anodes are all parallel to each other. This means that the grating disperses the spectrum in the same direction that the spatial imaging occurs. It is only the presence of the exit slit that selects the desired wavelength to be observed. The spatial resolution is determined by the anode-to-anode separation and by the distance from the exit slit to the front of the detector. Moving the detector closer to the exit slit will decrease the spatial resolution and will correspondingly increase the total field of view (as long as other optical components are not overfilled). The small dimension of the slits, which determines the spectral resolution, must also be considered in the design of this system. Defocusing will occur if the slit size increases and approaches the anode width and also if the slit width is so small that diffraction effects become important. This means that the grating spacing must be chosen to achieve the desired spectral resolution with slit sizes that do not degrade the focusing properties. The details of all these considerations are given in Appendix $C$.

\section{IV.4 INSTRUMENT LOCATIONS ON TMX}

Throughout this project, the main emphasis was placed upon studying the central cell of TMX; the single channel instrument and the LLNL-SIDS obtained extensive amounts of data from that region. The LLNL-SIDS was also mounted briefly on the east end cell, but most data from here was obtained with the JHU-SIDS. Limited amounts of central chord data were taken from the west end cell with the single channel instrument. This section covers chronologically the locations on TMX that were occupied by the various instruments.

The first instrument taken to TMX was the single channel instrument. It was mounted on the central cell and, after difficulties due to damage during shipment, the first data was taken on July 20, 1979. It was not possible to tilt this instrument for spatial profiles, so only central chord information was obtained. Nevertheless, it provided the first indication that the impurity densities were low in the central cell. Enough datc was taken to 
provide estimates of trends in central cell electron temperature (there was no Thomson scattering on the central cell at this time) with machine parameters. In April 1980, when the LLNL-SIDS was mounted on the central cell and the JHU-SIDS was mounted on the east plug, the single channel instrument was moved to the west plug. Enough data was taken on the west plug to ascertain that no major differences existed between the impurity levels there and those in the east plug, however, first priority was given to taking data with the two spatial imaging instruments.

In January 1980, the LLNL-SIDS was taken to TMX with $\mathrm{f} / 19$ optics and was mounted on the east plug. This period was intended to be primarily for the instrument shakedown; data was taken only from January 23 to February 8. Spatial profiles of oxygen and $L y_{\alpha}$ were obtained, but basically no study was performed.

During February and March of 1980, the LLNL-SIDS was returned to JHU and its optics were enlarged to $f / 11$ to be more compatibl? with the larger central cell plasma radius. In March, it was returned to TMX, was mounted on the central cel1, and took data there from April 18 until TMX shutdown on 0ctober 4, 1980. This provides the bulk of the data used in this report. Many spatial profiles were taken of the central cell impurity brightnesses and the density levels obtained confirmed the low levels indicated by the single channel instrument.

The JHU-SIDS was also taken to TMX in March which was the first time for this instrument. It was mounted on the east plug and took data from March 27, 1980, until TMX shutdown. Unfortunately, an intermittent noise problem plagued this instrument after only a few weeks of operation. After three months of searching for sources of noise, the signal levels returned to normal, but the spatial profiles were more sharply peaked than those measured. during the first few weeks. During disassembly of TMX, it was discovered that two waveguides for microwave line-density measurements had been installed in the field of view of the instrument. Therefore, most of the radial profiles obtained from the east plug with the JHU-SIDS cannot be used, although the central chord brightnesses are assumed to be correct for the data taken when the noise problem was not present.

In summary, the majority of data taken for this experiment was obtained on the central cell, first with the single channel instrument and then with the LLNL-SIDS. Central chord brightnesses were obtained on the east plug with the JHU-SIDS and sufficient data was obtained from the single charinel 
instrument on the west plug to ascertain that no major differences existed between the impurity densities in the two end cells. The final locations of the three instruments are shown in Fig. IV.4; all were mounted above TMX and viewed vertical chords of the plasma. 


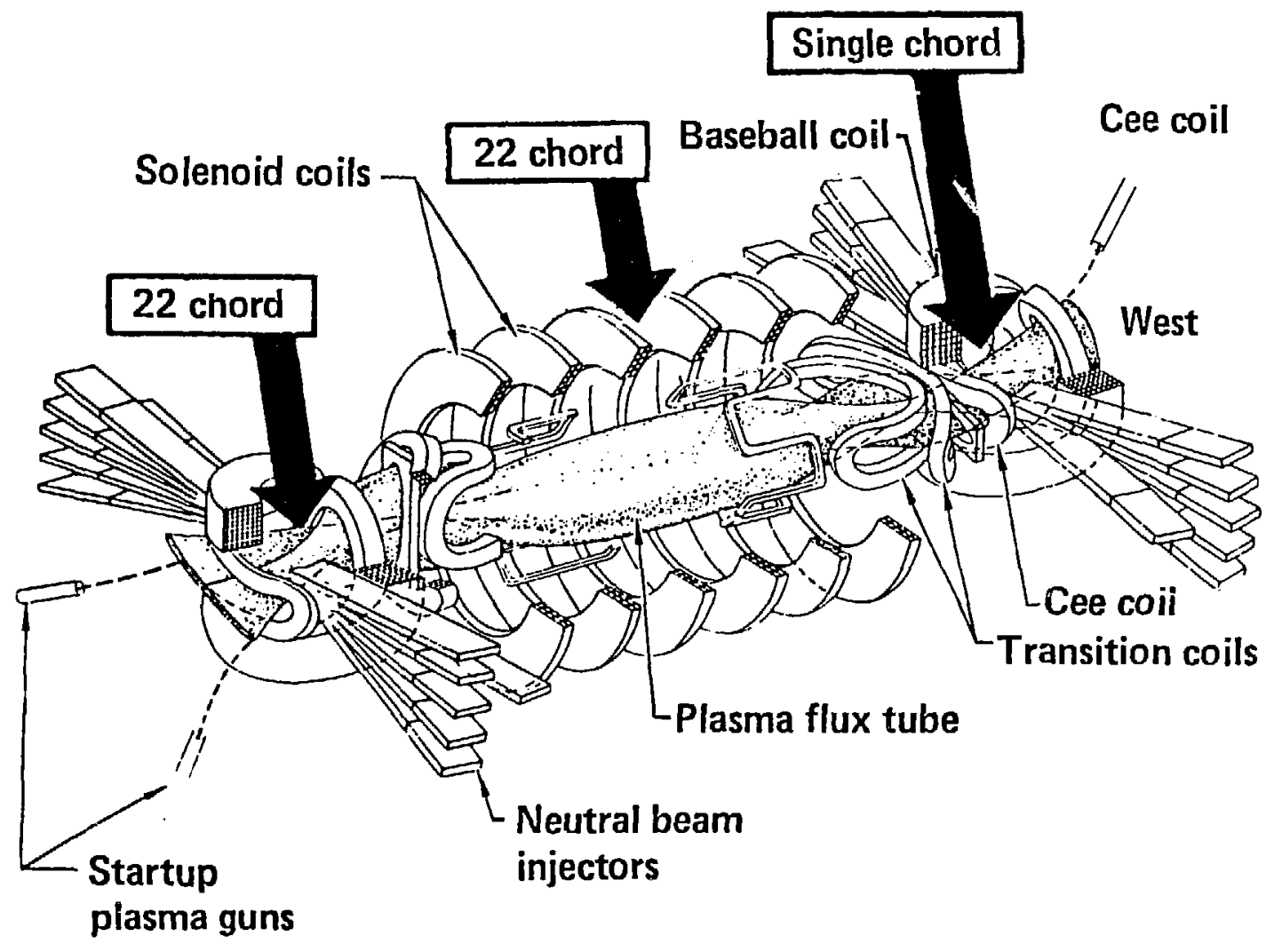

Fig. IV.4. Final locations of the three EUV instruments on TMX. 


\section{INTRINSIC IMPURITY BRIGHTNESSES AND CONCENTRATIONS}

V.1. INTRODUCTION

TMX provided a set of plasma conditions which are quite different from those observed on tokamaks. The electron temperatures ranged frain $50 \mathrm{eV}$ to $100 \mathrm{eV}$ on axis, and varied slowly with radius. The ion temperatures in the central cell were generally 50-100 eV, while in the end cells, $10 \mathrm{keV}$ ion energies were observed, so that Doppler broadening of the emission lines was quite pronounced in the end cells. Qualitatively different radial profiles of impurity emissions resulted depending on whether the gas boxes or puffer valves (see Chapter II) were used to fuel the central cell plasma.

The formalism applied to the data presented here was developed in Chapter III, so only the results will be given in this chapter. Most of the running on TMX used gas box fueling of the central cell; therefore, the results quoted for brightnesses, impurity densities and radiated power losses are for gas box operation. Section 2 gives a qualitative overview of the general characteristics of the impurity emissions, including the impurities and brightnesses observed, temporal and spatial profiles of the impurity emissions and coppler broadening observed in the end cell emissions. Section 3 presents the impurity concentrations; it also discusses metastable ratio measurements and possible contributions to the total impurity densities from the unobserved He-like species. Section 4 calculates the radiated power losses due to the intrinsic impurities. Finally, Section 5 considers possible sources of impurities in the plasma.

\section{V.2. GENERAL CHARACTERISTICS OF IMPURITY EMISSIONS}

\section{V.2.1 Impurities Identified}

The major impurities identified in TMX by this study were carbon, nitrogen, oxygen and titanium. (That no other abundant impurities were present in the plasma was verified by R. J. Fortner, T. Nash, and D. Dietrich of LLNL, who performed a photographic survey from $50 \AA$ to $6000 \AA$ using both a grazing incidence and normal incidence spectrograph [21].) The light impurities--carbon, nitrogen, and oxygen--were studied most carefully with the three monochromators described in Chapter IV. The resonance transitions of 
C II-IV, N II-V, 0 II-VI, as well as transitions to the low-lying metastable levels of C III, N IV, 0 II, 0 III, 0 IV, and 0 V were measured. Ground state transitions of Ti III, IV, VI, VII, IX, $X$ were also observed. Table V.I lists the transitions observed in the east end cell and central cell of TMX.

\section{V.2.2 Temporal Profiles}

As described in Chapter II, a warm deuterium plasma is injected along field lines into the end cells to provide a target for the neutral beam injectors. The plasma density builds up in each end cell and then flows into the central cell ionizing the fuel gas in that region. Figure V.l shows the deuterium $L y_{\alpha}$ brightness from the east end cell as a function of radius and time during a single TMX shot. The emissions from the target deuterium plasmas can be seen from $5 \mathrm{~ms}$ to $10 \mathrm{~ms}$ which is when the start-up guns were fired on this shot. After the start-up guns are turned off, the plasma quickly reaches a steady state which is maintained until the neutral beams are turned off at the end of the TMX shot. Figure V.2 shows examples of temporal profiles of oxygen emissions from both the east end cell and the central cell. Only the central chord emissions are shown so that the effect of the start-up plasmas is smitted. The time required for the end cell plasmas to flow into the central cell is observed in the delay of the initial increase in the impurity emissions from the central cell. The emissions are generaliy free from large fluctuations and maintain a constant value throughout the shot. This behavior is typical of the emissions from all other impurities observed. Their representative values of steady state brightnesses observed from the TMX central cell and east end cell are presented in Table V.l.

\section{V.2.3 Spatial Profiles}

Brightness profiles outained from the central cell are emphasized in this report; the radial profiles obtained from the east end cell were qualitatively similar to those observed on the earlier $2 X I I B$ experiment [16]. The sing?yand doubly-ionized states show approximately constant density with radius and the higher ionization states are more appropriately described as having constant concentrations. Because of the limited number of radial profiles obtained from the TMX end cells, the $2 X I I B$ radial profiles with the TMX end 


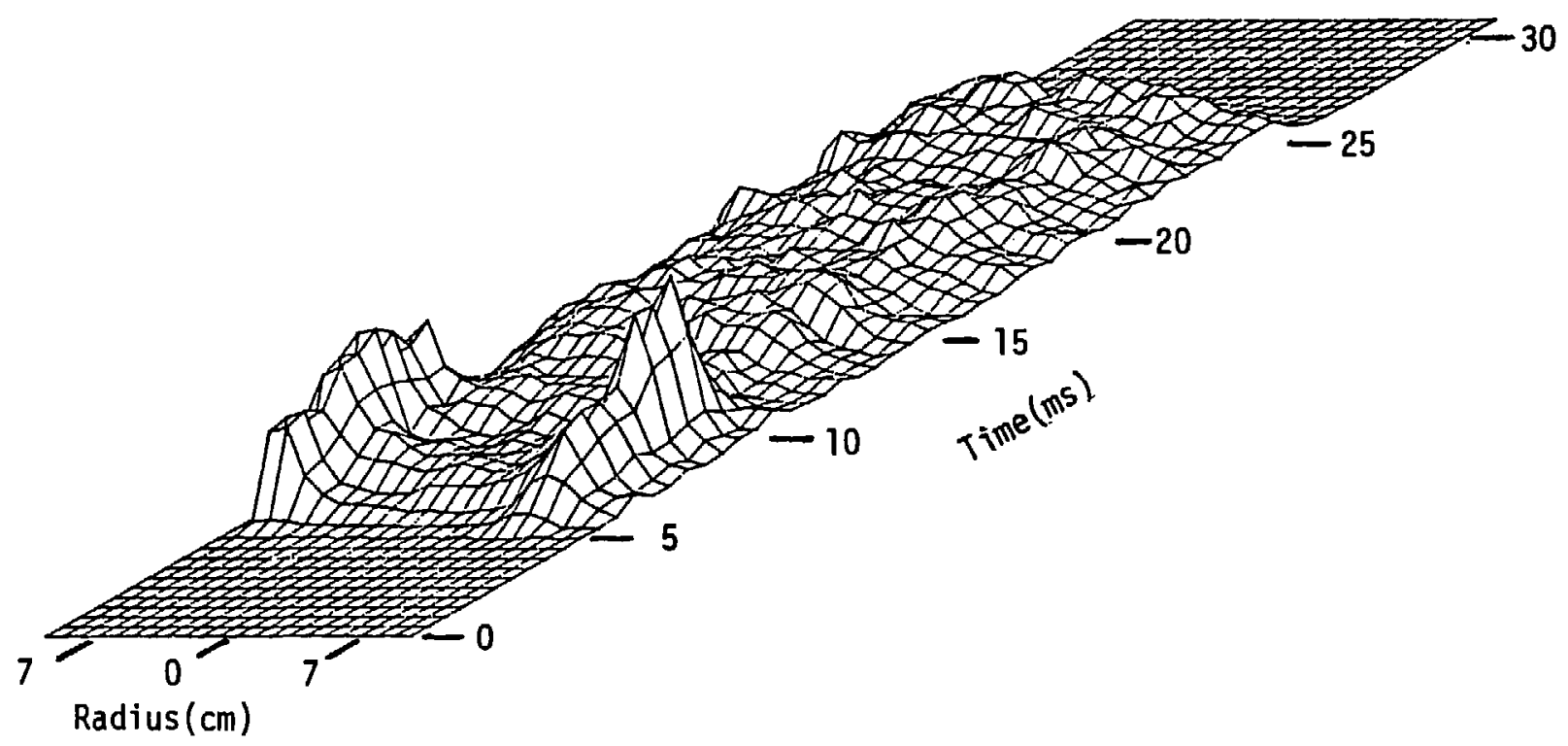

Fig. V.1. Temporally and spatially resolved brightness of deuterium $L y_{\alpha} 1215 \AA$ from the east end cell of TMX. Note the effect of the start-up gun plasmas. 

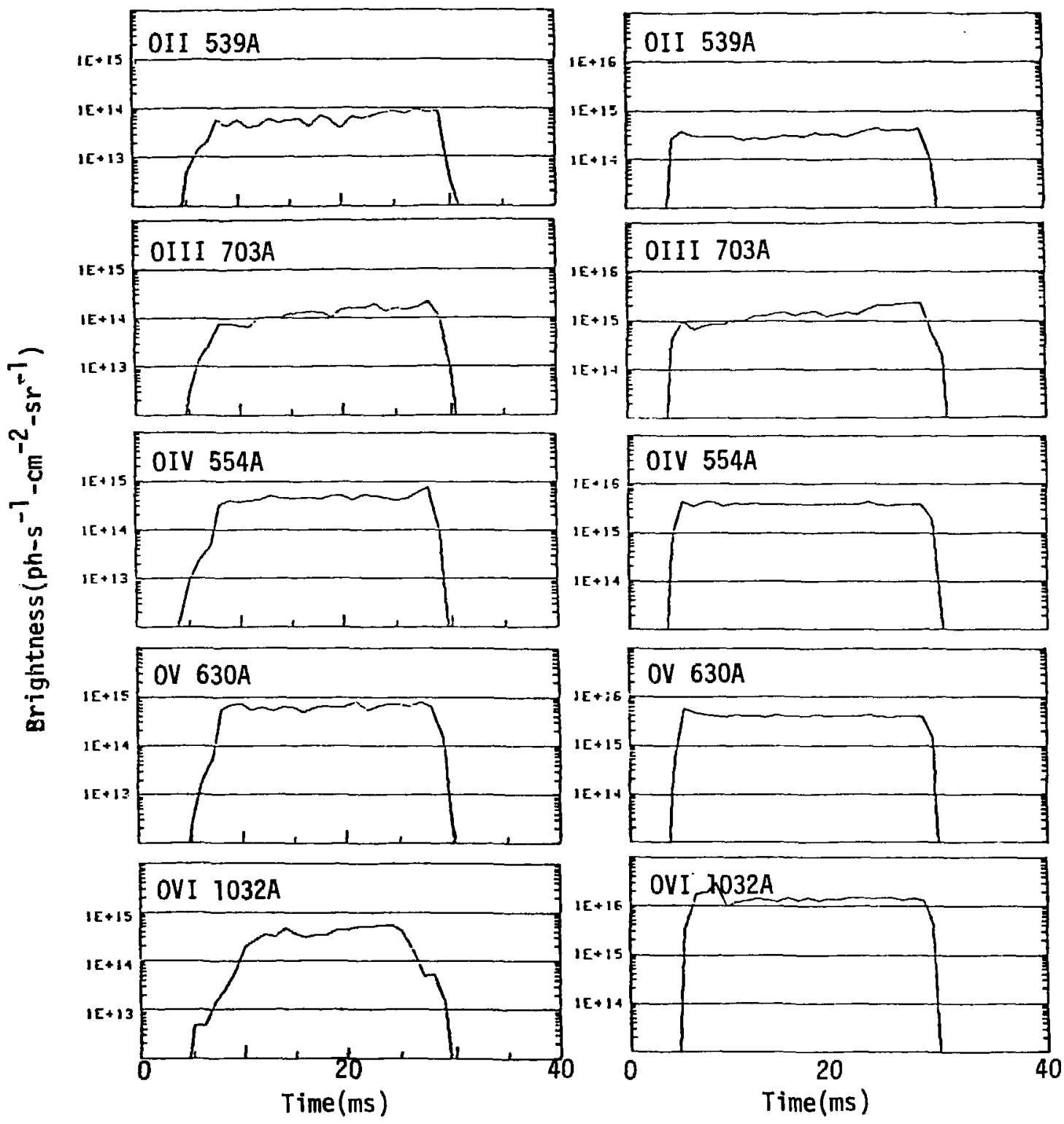

Fig. V.2. Temporal profiles of oxygen ion brightnesses from the central cell and east end cell of TMX. These are central chord profiles which do not include the effects of the start-up gun plasmas. 


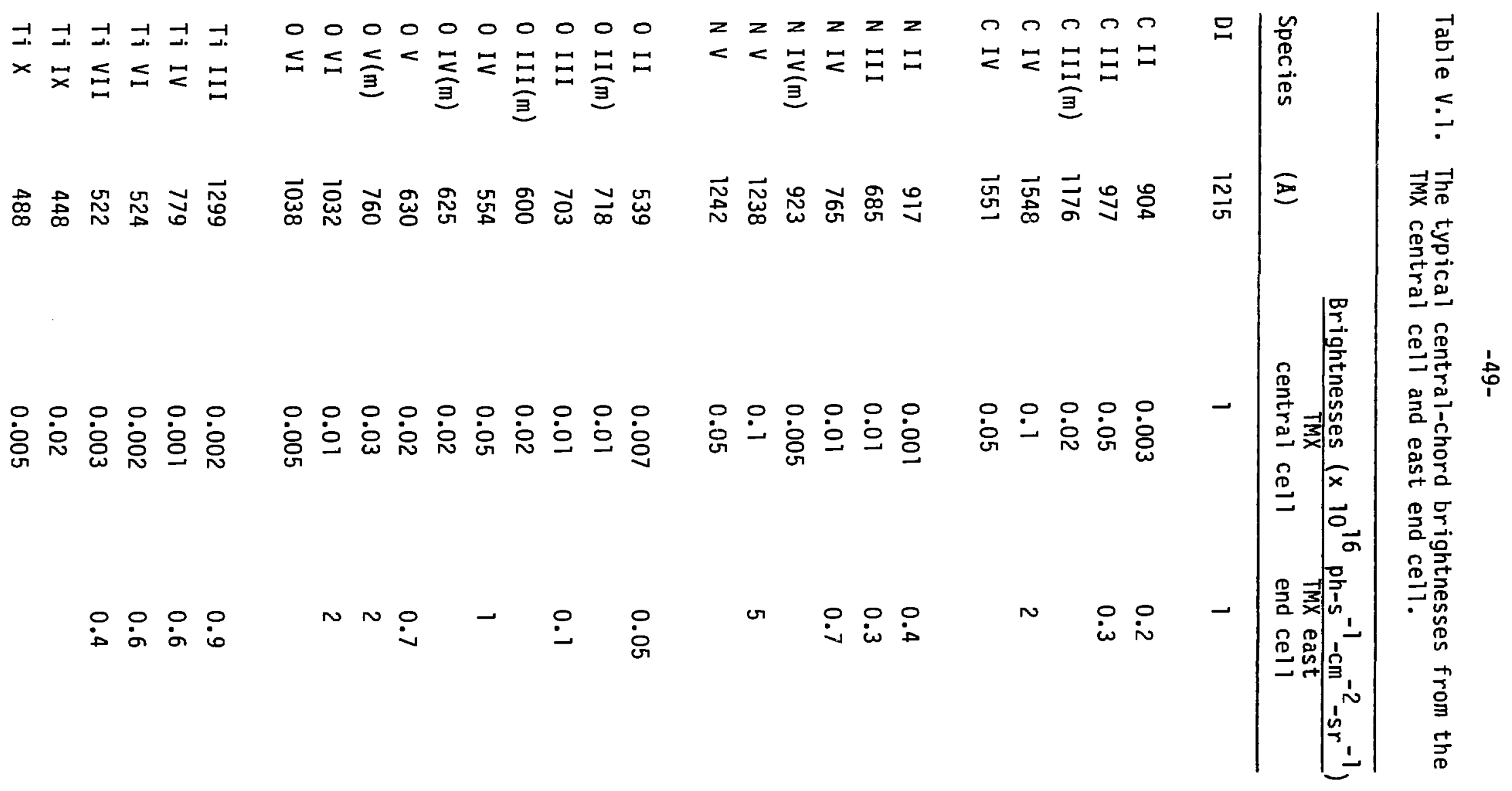


cell central chord measurements were used to calculate the impurity concentrations in the TMX end cells (Section V.3).

In the central cell, the shape of the radial brightness profiles depends upon whether the plasma is fueled with the gas boxes or with the puffer valves. During gas box operation, the steady-state radial brightness profiles of all observed impurity ionization states have a maximum along the central chord and decrease toward the plasma edge. Figure V.3 shows the brightness profiles of 0 II through 0 VI; full radial scans of both halves of the plasma indicate that the brightness profiles are generally symmetric. The shapes of these profiles are similar to the radial profiles of the electron line density as shown. This behavior is observed in the profiles of the other impurity species also. The similarity in shape between the ion brightness profiles and the electron line densities and the discussion in Section III.3.3 indicate that the ion density profiles are approximately constant with radius.

During puffer valve fueling, the central cell impurity brightness profiles are broader. The brightnesses of all observed ionization states of each impurity remain nearly constant from the central chord to about $35 \mathrm{~cm}$ in chordal position and then drop rapidly to zero somewhat beyond the limiter. Figure V.4 shows that the radial brightness profiles of NII, N V, $0 I I$, and 0 VI are all similar; the electron line density profiles are also shown to be nearly constant with radius but start to decrease at a chord position of about $30 \mathrm{~cm}$. This indicates that the impurity ion densities are nearly constant in the central plasma, as in the gas box case, but then increase toward the plasma edge. Full radial scans generally show symmetric impurity brightness profiles for the case of puffer fueling also.

The Lyman alpha brightness profiles showed more variations than the impurity profiles; asymmetries were often observed and were believed to be caused by asymmetries in the fueling. Figure V.5(a) shows a symmetric brightness profile obtained during gas box operation. As is typical for the impurity brightness profiles with gas box operation, the Lyman alpha profile also peaks along the central chord. The electron line density profile, however, is narrower which indicates that the neutral density peaks at the plasma edge. Figure V.5(b) shows the neutral density profile which was obtained from the matrix inversion (Section III.2) of the brightness profile and Eq. (III.4). The electron density profile was measured to be Gaussian (peak density of $4.7 \times 10^{12} \mathrm{~cm}^{-3}$ with an e-folding width of $26 \mathrm{~cm}$ ) by the beam attenuation diagnostic [22]. The electron temperature was measured to be 


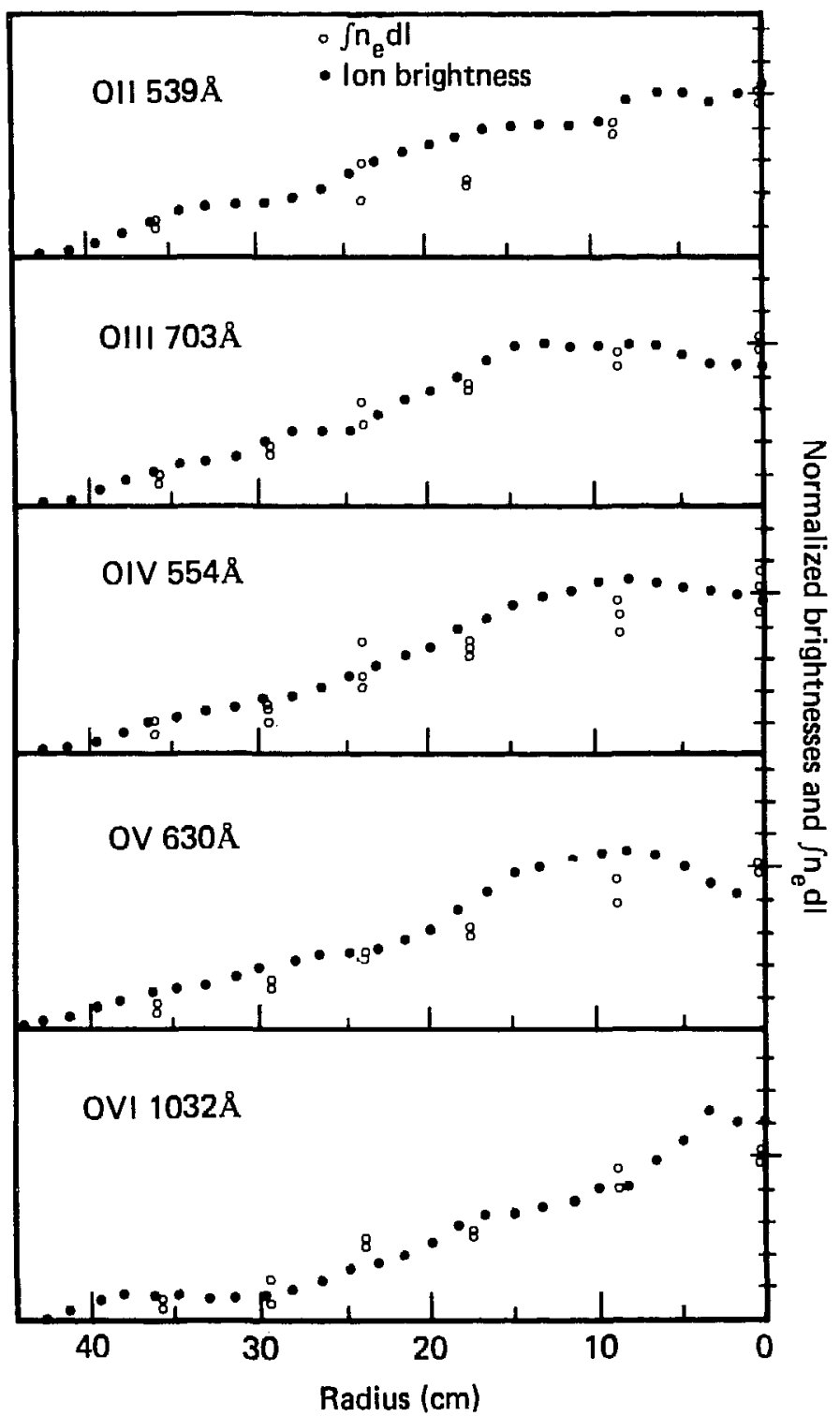

Fig. V.3. Central cell radial brightness profiles of 0 II - 0 VI and the corresponding electron line density profiles during gas box fueling of the central cell plasma. 


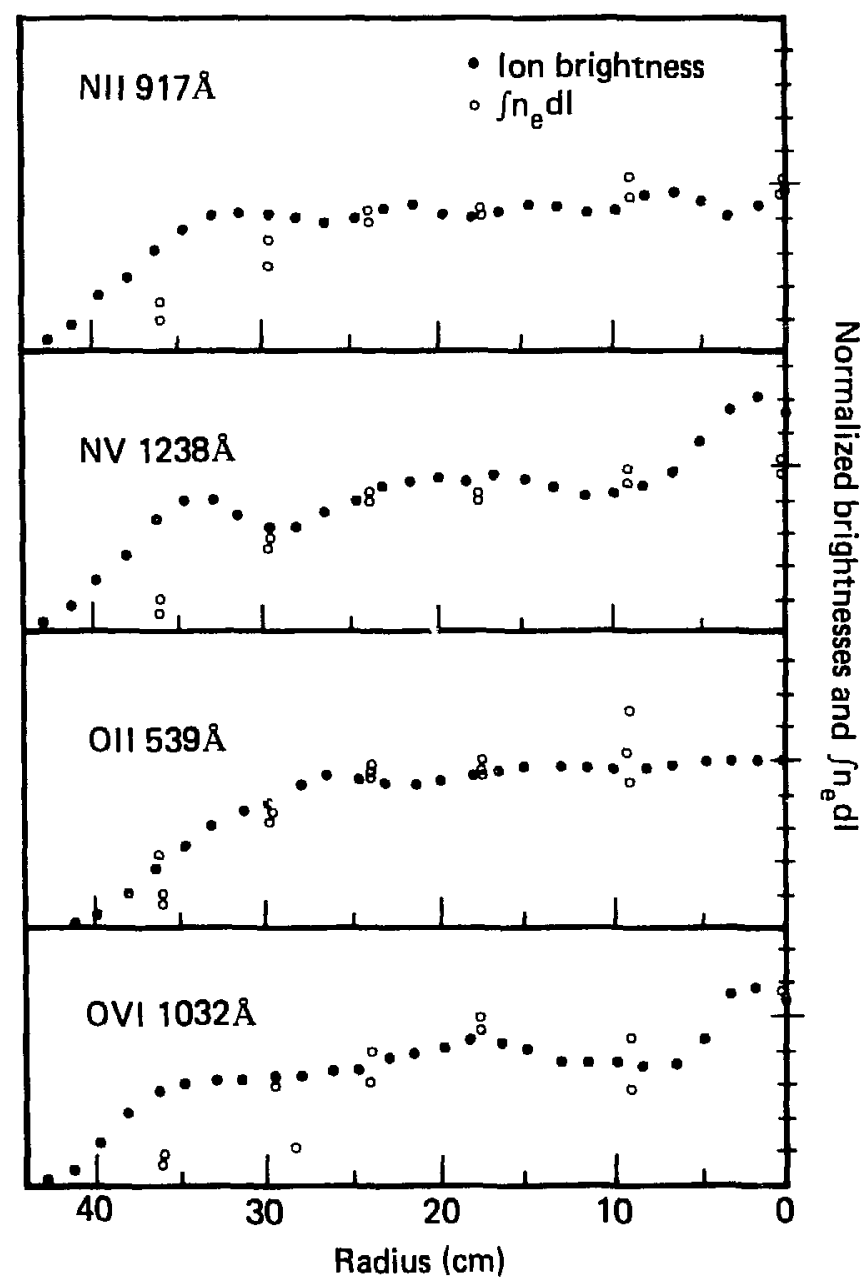

Fig. V.4. Central-cell radial brightness profiles of $N$ II, N V, 0 II, $0 \mathrm{VI}$ and the corresponding electron line density profiles during puffer fueling of the central cell plasma. 

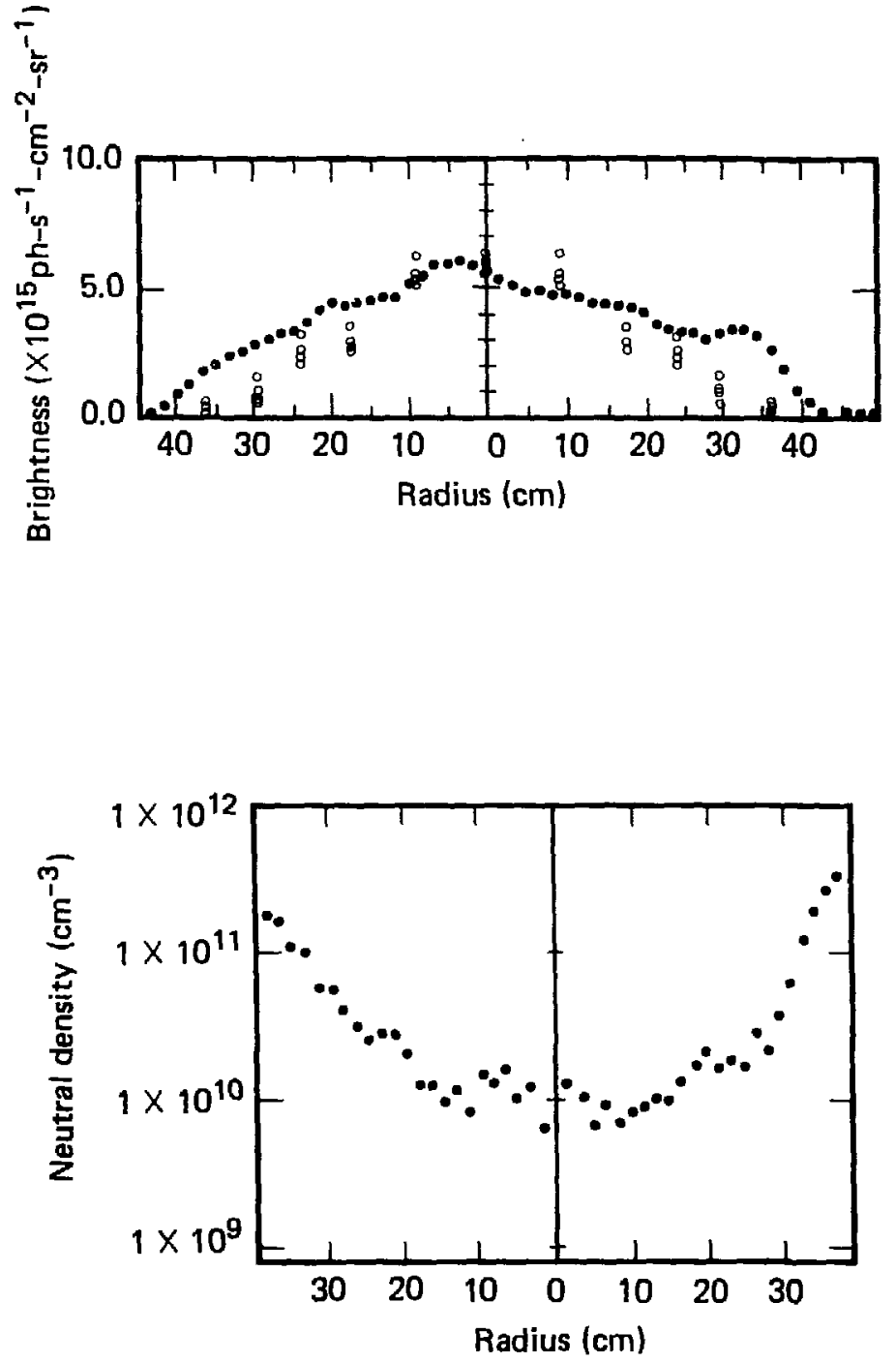

Fig. V.5. Central-cell radial profile of (a) deuterium $L y_{\alpha}$ $1215 \mathrm{~A}$ brightness and (b) the corresponding neutral density. 
$43 \mathrm{eV}$ on axis hy Thomson scattering and was assumed to be Gaussian with radius such that the temperature at the limiter was $10 \mathrm{eV}$ as discussed in Section v.3.1.4. The excitation rate coefficient varied with temperature as shown in Fig. A.4. The neutral density drops a factor of 20 to 40 from the edge to a central density of about $1 \times 10^{10} \mathrm{~cm}^{-3}$; this is about $2.5 \times 10^{-3}$ of the electron density on axis.

\section{V.2.4 Doppler Widths of EUV Emissions}

Ion temperatures in the central cell were less than $200 \mathrm{eV}$ so that Doppler broadening is negligible compared to the spectral resolution of the monochromators. Figure V. 6 shows a spectral scan of the 0 VI doublet at $1032 \AA$ and $1038 \AA$ taken by the single channel instrument in the central cell. The spectrai width of each line is essentially determined by the 2 \& resolution of the instrument. Note also the $2: 1$ ratio of intensities determined by the $(2 j+1)$ weighting of each 1ine. All other emission lines in the central cell also showed no observable Doppler broadening.

In the end cells, the neutral beam injectors provided ion energies on the order of 10-40 keV. Therefore, the emissions from impurities would be expected to show significant Doppler broadening. Figure $V .7$ shows the spectral profiles of $O V 630 \AA$ and N II $917 \AA$; the instrumental resolution was set at $1 \AA$. Table V.2 lists the Doppler energies observed from spectral scans taken in the east end cell; these vilues are obtained using Eq. (III.8). The primary purpose of these spectral scans was to locate the wavelength settings on the monochromators corresponding to the peak of the emissions. Therefore, wavelength step sizes taken while scanning the lines were generally on the order of $1 \mathrm{~A}$, which is too large for a careful determination of the ion enc gies. An uncertainty of $\pm 0.5 \AA$ in the width of an emission line at, say, $1000 \AA$, corresponds to an energy of about $2 \mathrm{keV}$. For transitions at shorter wavelengths, this given step size in the spectral scar results in even larger uncertainties in the derived energies. Shot-to-shot variations also increase the uncertainties, so that factors of 2 or 3 incertainties are probably not unreasonabie. In spite of the uncertainties involved, several results may be noted. Nitrogen and oxygen have energies on the order of 10-30 kev, while carbon has a much lower energy. This provides evidence for the possible sources of impurities in the end cells, as will be discussed in Section V.5. (It was further noted that the ion energies increased with $Z$ for 


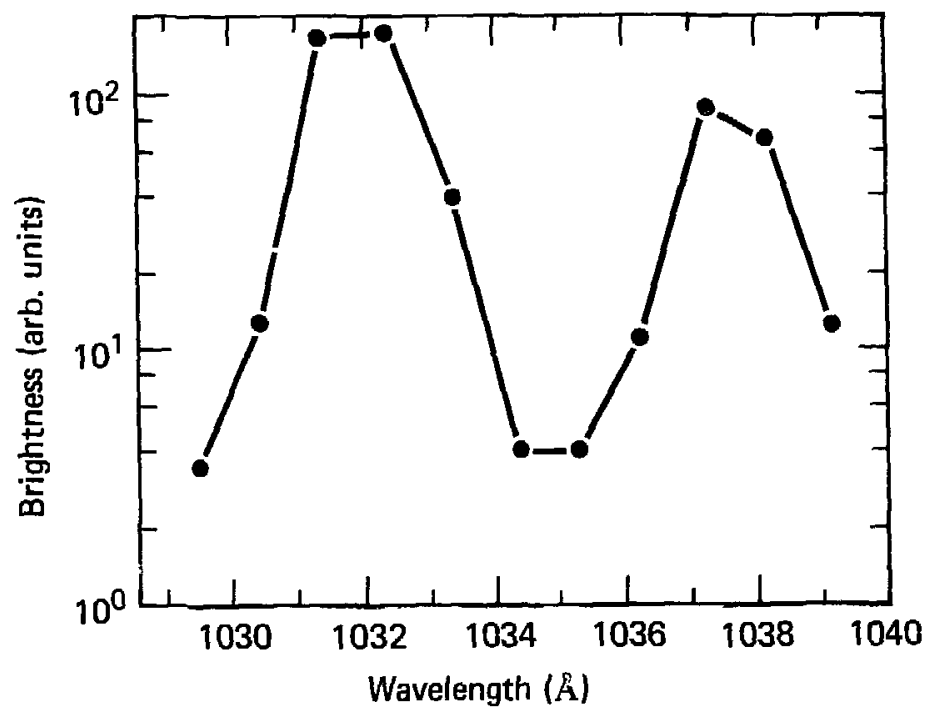

Fig. V.6. Spectral scan of O VI doublet at $1032 \AA$ and $1038 \AA$ from TMX central cell. The line widths are determined by the $2 \AA$ instrumental resolution. 

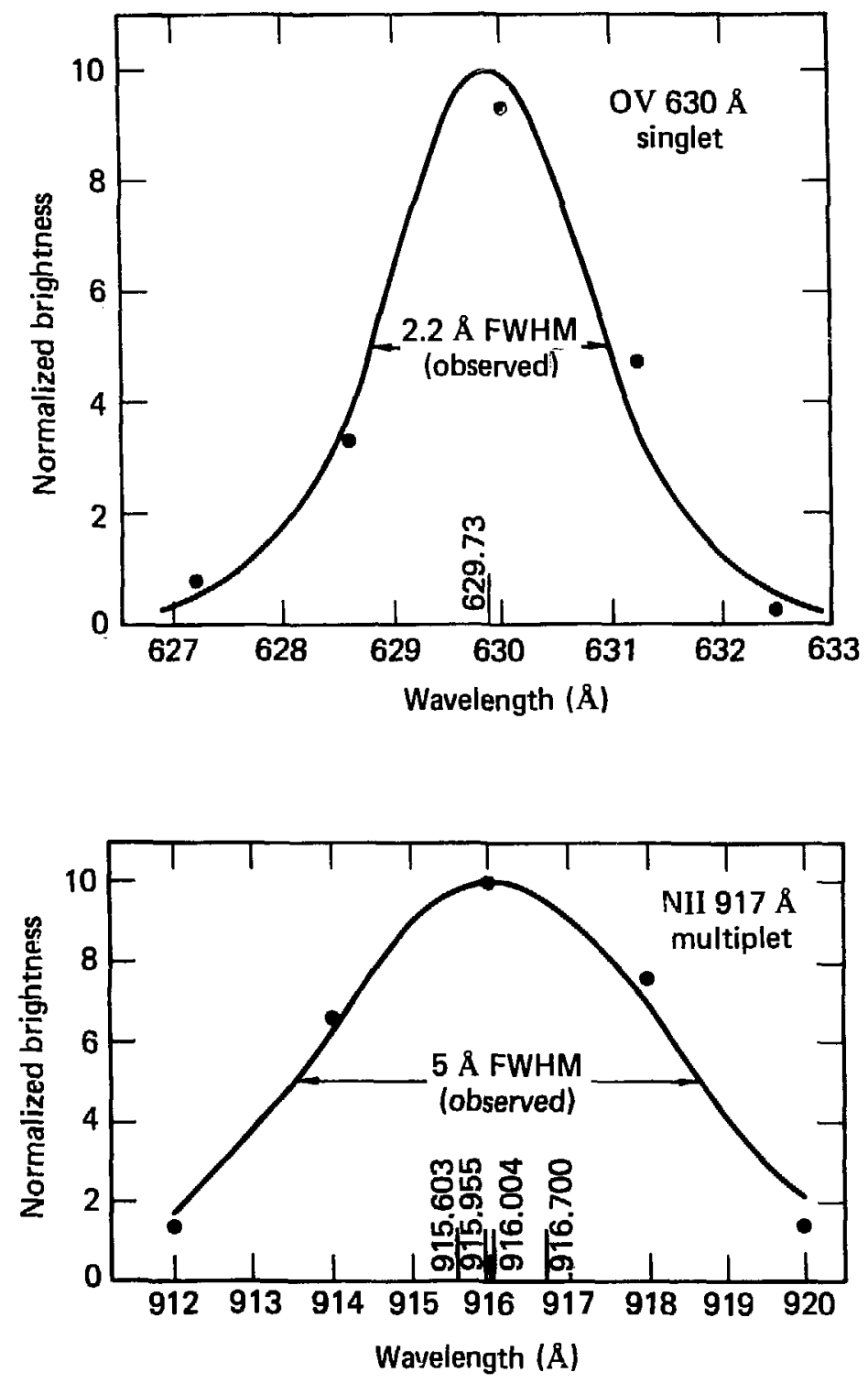

Fig. V.7. Spectral scans from TMX east end cell showing Doppler broadening of (a) 0 V $630 \AA$ and (b) N II $917 \mathrm{~A}$. The instrumental resolution is $1 \mathrm{~A}$. 
Table V.2. Ion energies in east end cell obtained from Doppler broadening measurenents.

\begin{tabular}{lrlr}
\hline Date & Shots & Ion/wave length (A) & E (keV) \\
\hline $7 / 16 / 80$ & $14-24$ & C IV 1548 & 3 \\
$7 / 18 / 80$ & $4-11$ & N II 91/ & 26 \\
$5 / 29 / 80$ & $21-31$ & 0 III 703 & 10 \\
$5 / 29 / 80$ & $61-66$ & 0 V 630 & 23 \\
$5 / 29 / 80$ & $44-50$ & 0 VI 1032 & 30 \\
$2 / 5 / 80$ & $18-31$ & Ti VI, V II 522 & 80 \\
& & & \\
\hline
\end{tabular}


0 III, $0 \mathrm{~V}$, and $0 \mathrm{VI}$. Whether this is a consequence of the uncertainties in the measurements or represents a real effect is not known.)

\section{V.3. IMPURITY CONCENTRATIONS IN TMX}

\section{V.3.1 Light Impurities}

V.3.1.1 Method. The direct method for calculating the total density of a given impurity is to determine the density of each ionization state in the plasma. The radial brightness profile of a transition to the ground state and any highly-populated metastable state of each species must be measured and inverted to obtain the volume emission rates. Dividing by the measured electron density profiles and calculated excitation rates then yields the density of each ionization state [Eq. (III.4)]. The total density is obtained from the sum of the densities of all ionization states. This is the method used to determine the total impurity densities for carbon, nitrogen, and oxygen in TMX. The following sections discuss the metastable densities for the various ionization states, the question of the He-like densities expected, and the electron temperature profiles assumed for these calculations. The final section of the light impurity discussion presents the results.

\section{V.3.1.2 Metastable Densities. Some ionization states have low-lying} metastable levels from which radiative de-excitation to the ground state is optically forbidden. Depopulation of these metastable levels occurs mainly through collisional processes--de-excitation to the ground state or excitation to a level in the ground state system. In TMX and other magnetic plasma devices, the densities are so low that these collison rates may be much slower. than radiative de-excitation to the metastable levels, therefore, a significant fraction of the total ion density may exist in one or more metastable levels in addition to the ground state. Therefore, in order to determine the total ion density, the brightness of transitions from these metastable levels must be measured or the metastable density must be estimated. Table V.3 lists the ratios of metastable density to ground state density used in this study to calculate the total impurity densities. Transitions to the lowest-lying metastable levels of all ionization states of oxygen were measured in the central cell. The metastable transition of $0 \mathrm{~V}$ was measured in the east end cell, but the metasiable ratios for the other 
Table V.3. Ratios of metastable to ground state density $\left(n_{m} / n_{g}\right)$ used to calculate total impurity densities and radiated power losses.

\begin{tabular}{lll}
\hline Species & Central Cell & End CeITS \\
\hline C I I & 0.5 & 0.5 \\
C I I & 0.7 & 2 \\
C IV & 0 & 0 \\
& & \\
N I I & 1.5 & 1.5 \\
N I I I & 0.5 & 0.5 \\
N IV & 0.7 & 2 \\
N V & 0 & 0 \\
0 I I & 0 & \\
O I I & 1.5 & 0 \\
O IV & 0.5 & 0.5 \\
O V & 0.7 & 2 \\
O VI & 0 & 0 \\
\hline
\end{tabular}


ionization states of oxygen in the end cell were taken to be the same as those in the central cell. The metastable transitions of beryllium-like carbon and nitrogen were also measured in the central cell. All other metastable ratios were assumed to be equal along iso-electronic sequences from the values for oxygen in both the end cell and central cell.

V.3.1.3 He-like Densities. Coronal equilibrium calculations which balance ionization and recombination rates $[23,24]$ predict that nearly all of the density of carbon, nitrogen, and oxygen should exist in the helium-like ionization state at an electron temperature of $50 \mathrm{eV}$. Emissions from these He-like states are outside the wavelength range of the instruments described in Chapter IV and could not be observed during this study, so it is possible that a large fraction of the total impurity density was not included in these measurements. However, the fractional abundances of the observed ionization states in the centra? cells indicate that this is not the case. Table V.4 gives the relative densities of the oxygen ionization states determined from the typical brightnesses given in Table V.l. The highest fraction is contained in the boron-like state while higher ionization states have decreasing amounts. Section V. 6.3 presents results of a code which calculates confinement-modified steady-state ionization equilibrium. As discussed in Section V.6.3, these results for the case of oxygen indicate that helium-like 0 VII comprises approximately $6 \%$ of the total oxygen density with a $1 \mathrm{~ms}$ conf inement time. A similar result would be expected for carbon and nitrogen. Therefore, it is assumed that the observed ionization states of the light impurities account for nearly $100 \%$ of the densities.

\section{V.3.1.4 High Electron Temperature at Edge of Central Cell Plasma. Inverting} the impurity brightness profiles while calculating ion densities requires a high electron temperature at the plasma edge in order to obtain physically reasonable radial density profiles. If the electron temperature profiles are given the same width as the measured electron density profiles, the ion densities often appear to increase over an order of magnitude at the plasma edge for all ionization states. The cause of this apparent increase is due to the rapid decrease of the excitation rate coefficients below $10 \mathrm{el}$ (see Appendix A). It is physically unreasonable for the densities of highly ionized impurities to increase in a region where the electron temperature should be low. Therefore, the shape of the ion radial profile becomes very 
Table V.4. Ionization state distribution observed for oxygen in the central cell of TMX.

\begin{tabular}{lll}
\hline Species & Z & Fraction \\
\hline 0 II & $?$ & 0.13 \\
0 III & 2 & 0.16 \\
0 IV & 3 & 0.44 \\
0 V & 4 & 0.22 \\
0 VI & 5 & 0.05 \\
\hline
\end{tabular}


sensitive to electron temperature at the plasma edge. Widening the temperature profiles in order to raise the temperature to $10-15 \mathrm{eV}$ at the limiter $(38 \mathrm{~cm})$ results in radial density profiles which are more reasonable. Assuming a constant electron temperature with radius causes almost no further change in the ion density radial profiles because the excitation rates become insensitive to temperature above $10 \mathrm{eV}$. This justifies the use of a constant electron temperature in the central cell for the ion concentration calculations. Therefore, the data puts a lower limit of 10-15 eV on the edge electron temperature in the central cell.

The presence of highij-ionized species near the plasma edge is apparently due to an outward radial transport of these species in times which are short compared to recombination times (Chapter VI). An edge temperature of $10 \mathrm{eV}$ is not high enough to create high charge states by ionization, but is sufficient to excite transitions of those charge states so that they radiate at the low temperatures near the plasma edge.

V.3.1.5 Results. Table V.5 presents the average concentrations obtained for carbon, nitrogen, and oxygen in the TMX central cell and east end cell. Insufficient data was taken in the west end cell to actually calculate concentrations, however, brightnesses observed there were similar to those in the east end cell. This indicates that the impurity concentrations in the west end cell were comparable to those in the east.

In the central cell, two methods are used to estimate the impurity concentrations. The direct method is to perform a matrix inversion of the brightness profiles and then to divide by the corresponding electron density profiles and electron impact excitation rates [Eq. III.4]. These density profiles are integrated numerically over the volume of the central cell to obtain the total number of impurity ions. Finally, these totals are divided by the total number of electrons in the central cell lobtained by similar integrations) in order to yield the volume average concentration of each impurity ion. The second method is to assume that the ion density profiles are constant with radius and to use the formalism developed in Section III. 3. The results of these two methods differ by no more than $35 \%$ for the total concentration of each impurity; the values presented in Table V.5 were obtained from the second method. The light impurity concentrations are less than $0.4 \%$ each; using on?y the light impurities to calculate $Z$ eff implies $Z_{\text {eff }} \sim 1.07$. In the east end cell, only the central chord brightnesses and 
Table V.5. Average impurity concentrations in the TMX central cell and east end cell. The low-impurity concentrations in the central cell yield $Z_{\text {eff }} \sim 1.1$.

\begin{tabular}{ccc}
\hline $\begin{array}{c}\text { Impurity } \\
\text { concentrations (\%) }\end{array}$ & $\begin{array}{c}\text { TMX } \\
\text { central cell }\end{array}$ & $\begin{array}{r}\text { TMX east } \\
\text { end cel1 }\end{array}$ \\
\hline Carbon & 0.08 & 0.3 \\
Nitrogen & 0.1 & 0.7 \\
Oxygen & 0.4 & 1.5 \\
Titanium & 0.1 & 0.3 \\
\hline
\end{tabular}


the models described in Section III.3 are used to calculate the concentrations; the concentration is higher for each impurity than that observed in the central cell. $Z_{\text {eff }}$ in the east end cell is approximately 1.4 , again considering only the light impurities.

\section{V.3.2 Titanium}

V.3.2.1 Method. A different approach is taken to determine the titanium concentrations in TMX since not all of the expected ionization states have transitions in the wavelength range of the monochromators. Furthermore, the needed excitation rate coefficients are not available in order to perform the density calculations. The method used for titanium is to estimate the density of a single ionization state from its observed brightness with the constant density model and then use the results of coronal equilibrium calculations to estimate what fraction of the total titanium density existed as the observed stati. In the central cell, the observed transition is Ti IX $448 \AA$, and in tast plug, Ti VII $522 \AA$ is used. The next section explains how the excitation rates are obtained, Section 3.3.3 gives the ionization state distributions used, and Section 3.3.4 presents the results.

\section{V.3.2.2 Excitation Rates. The excitation rates for Ti VII $522 \AA$ and for} Ti IX $448 \AA$ were not found in the literature, but were obtained by interpolating oscillator strengths of the same transitions in other ions along the same iso-e lectronic sequences. $T i$ VII is in the sulfur-like sequence and $T i$ IX is in the silicon-like series.

Oscillator strengths for the S-like and Si-like ions of silicon, phosphorus, sulfur, chlorine, and argon were obtained from [25] and [26]. Oscillator strengths for the S-like and Si-like iron ions may be approximated from [27] which gives a set of $\alpha$ and $\beta(=\Delta E)$ coefficients to substitute directly into

$$
Q=\frac{\alpha e^{-\beta / T}}{T / 2} ;
$$

the oscillator strengths for the iron ions were obtained from the $a$ coefficients using Eq. (A.1) and assuming eoual average Gaunt factors [28] along each iso-electronic sequence. Table $y$. shows the results obtained in this manner; a value of $Q=9 \times 10^{-9} \mathrm{~cm}^{3} / \mathrm{s}$ was obtained for Ti VII $522 \AA$ and a value of $Q=5 \times 10^{-9} \mathrm{~cm}^{3} / \mathrm{s}$ was obtained for Ti IX $448 \AA$. 
Table V.6. Parameters used to calculate excitation rates for transitions of Si-like and S-like titanium.

Si-like:

Ti IX $448 \AA 3 s^{2} 3 p^{2}-3 s 3 p^{3} 3 p-{ }^{3} P_{0}$ S-like:

Ti VII $522 \AA 3 s^{2} 3 p^{4}-3 s 3 p^{5} 3 P-3_{0}$ \begin{tabular}{lllllllll}
\hline Species Sequence $\lambda(\AA)$ & $\Delta E_{j}(e V)$ & $f_{i k}$ & $\langle\bar{g}\rangle$ & $\alpha\left(\mathrm{cm}^{3}-e V^{1 / 2} / \mathrm{s}\right)$ & $B(e V)$ & $Q\left(\mathrm{~cm}^{3} / \mathrm{s}\right)$ \\
\hline
\end{tabular}

\begin{tabular}{lrrrrrrrr}
\hline Si I & Si & 1771 & 7.0 & 0.04 & 0.44 & & & $5 \times 10^{-9}$ \\
P II & Si & 1305 & 9.5 & 0.04 & 0.44 & & & $2 \times 10^{-9}$ \\
S III & Si & 1020 & 12.2 & 0.045 & 0.44 & & & $3 \times 10^{-9}$ \\
Ti IX & Si & 448 & 27.7 & 0.24 & 0.44 & & & $5 \times 10^{-9}$ \\
Fe XIII & Si & 321 & 41.9 & 0.37 & 0.44 & $6.19 \times 10^{-8}$ & 41.9 & $4 \times 10^{-9}$ \\
& & & & & & & & \\
CI II & S & 1071 & 11.6 & 0.02 & 0.4 & & & $1 \times 10^{-9}$ \\
Ar III & S & 878 & 14.1 & 0.03 & 0.4 & & & $1.5 \times 10^{-9}$ \\
Ti VII & S & 522 & 23.8 & 0.41 & 0.4 & & & $9 \times 10^{-9}$ \\
Fe XI & S & 315 & 39.4 & 0.84 & 0.4 & $1.36 \times 10^{-7}$ & 39.4 & $9 \times 10^{-9}$ \\
& & & & & & & & \\
\hline
\end{tabular}


v.3.2.3 Ionization Equilibrium. In order to determine the total titanium density from the density of one ionization state, the fraction of the total which that state comprises must be known. Ionization equilibrium calculations by Hulse [29] and by Breton et al. [30] which balance ionization and recombination were used to estimate the charge state distribution of the titanium ionization states. For the central cell case, the relative abundances at $50 \mathrm{eV}$ were used; Table V.7 shows the charge state distributions obtained from the two references. One indicates that Ti IX makes up $34 \%$ of the total titanium density; the other indicates $13 \%$. The high neutral density in the end cells shifts the charge state distribution to lower ionization states at a given temperature. According to Hulse et al. [31], a neutral fraction of $10^{-4} n_{e}$ lowers the average charge state $\langle Z\rangle$ by about 2 . Calculating $\langle Z\rangle$ vs $T_{e}$ from [29] and [30] indicated that $\langle Z\rangle$ at $30 \mathrm{eV}$ is approximately 2 less than $\langle Z\rangle$ at $50 \mathrm{eV}$ in both cases. Therefore, the charge state distribution at $30 \mathrm{eV}$ was used to calculate the total titanium density in the east end cell. Table V.7 shows the ionization equilibrium given by the two references; one indicates that Ti VII is $42 \%$ of the total titanium density and the other indicates $13 \%$.

V.3.2.4 Results. Using the method described in the previous sections results in two different estimates for the total titanium density because two different ionization distributions were used. A lower bound for the total titanium density may be obtained by using only the measured transitions, an excitation rate of $5 \times 10^{-9} \mathrm{~cm}^{3} / \mathrm{s}$ for each transition, and assuming constant ion density radial profiles for each species. Therefore, three estimates of the total titanium concentration were obtained in the central cell $10.08 \%$, $0.1 \%, 0.2 \%)$ and three in the east end cell $(0.04 \%, 0.3 \%, 0.9 \%)$. The values quoted in Table V.5 correspond to the intermediate values. The three central cell estimates agree with each other within a factor of 2 of the intermediate value; the east end cell estimates show a wider range of uncertainty.

\section{V.4. RADIATED POWER LOSSES FROM TMX}

The light impurity radiated losses are calculated from the volume emission rates using the formalism described in Section III.4. Approximately $40 \%$ of the total estimated power loss was actually risured; the remainder included the contributions from over 40 other transitions which were scaled 
Table V.7. Ionization equilibrium distributions used to calculate total titanium density and radiated power loss.

\begin{tabular}{|c|c|c|c|c|}
\hline & Species & $Z$ & Hulse $[29,31]$ & Breton \\
\hline Central cell & $\begin{array}{l}\text { Ti } V \\
T i \text { VI } \\
\text { Ti VII } \\
\text { Ti VI II } \\
\text { Ti IX } \\
\text { Ti X } \\
\text { Ti XI }\end{array}$ & $\begin{array}{r}4 \\
5 \\
6 \\
7 \\
8 \\
9 \\
10\end{array}$ & $\begin{array}{l}0 \\
0.02 \\
0.15 \\
0.34 \\
0.34 \\
0.13 \\
0.02\end{array}$ & $\begin{array}{l}0.02 \\
0.13 \\
0.39 \\
0.30 \\
0.13 \\
0.03 \\
0\end{array}$ \\
\hline East end cell & $\begin{array}{l}\text { Ti } \text { IV } \\
\text { Ti V } \\
T i \text { VI } \\
T i \text { ViI } \\
T i \text { VIII } \\
\text { Ti IX }\end{array}$ & $\begin{array}{l}3 \\
4 \\
5 \\
6 \\
7 \\
8\end{array}$ & $\begin{array}{l}0 \\
0.07 \\
0.32 \\
0.42 \\
0.17 \\
0.02\end{array}$ & $\begin{array}{l}0.04 \\
0.38 \\
0.45 \\
0.13 \\
0 \\
0\end{array}$ \\
\hline
\end{tabular}


according to their excitation rates. The power losses from titanium are estimated from the total titanium densities obtained in Section V.3.3 and the resuits of cooling rate calculations by Post [32] in the central cell and by Hulse [31] in the east end cell. Those radiated losses are presented in Table V.8 for the central cell and east end cell.

The total radiated losses from the east end cell were approximately $0.6 \mathrm{~kW}$ and are therefore negligible; similar losses are assumed from the west end cell. The larger volume of the central cell radiated a total of approximately $25 \mathrm{~kW}$. A factor of two uncertainty in this result would allow as much as $50 \mathrm{~kW}$ to be radiated; this is still only about $10 \%$ of the net trapped neutral beam power $(\sim 400 \mathrm{~kW})$. Radiated power losses from TMX were a small fraction of the total power loss.

\section{V.5. SOURCES OF IMPURITIES}

\section{V.5.1 End Cell Sources}

Oxygen was observed to be injected by the neutral beams on 2XIIB [33], therefore, it is important to determine whether the neutral beams were a source of oxygen in the TMX end cells also. Spectra scans of the oxygen ionization states indicate ion energies of $10 \mathrm{keV}$ or more (see Table V.2) which is consistent with a beam injected source.

Additional evidence for beam-injected oxygen may be obtained by applying the model developed for $2 X I I B$ [16] to the TMX case. This model calculates the 0 II density by balancing the deposition rate into the plasma with the loss rate of 0 II due to ionization. The injected current of oxygen is assumed to be a constant fraction of the total beam current; furthermore, the production of 0 II from neutral oxygen via charge exchange and ionization is much faster than other processes so that the density of 0 II is a direct measure of the source of neutrals from the beams. According to the model then:

$$
f_{0} I_{b} A=\int n_{0 \text { II }} n_{e} s_{0 \text { II }} d V
$$

where $f_{0}$ is the fraction of oxvgen in the neutral beams, $I_{b}$ is the injected beam current, $A$ is the attenuation of the oxygen in the plasma with the transmission $T=\exp \left[-\sigma \delta n_{e} d \ell / 1.41\right]$ and $\sigma=4.32 \times 10^{-15} \mathrm{~cm}^{2}$ (includes electron and deuteron impact ionization and charge exchange with the ionized 
Table V.8. Radiated power losses from the TMX central cell and east erid cell.

\begin{tabular}{lcc}
$\begin{array}{l}\text { Radiated power } \\
\text { losses (kW) }\end{array}$ & $\begin{array}{c}\text { TMX } \\
\text { central cell }\end{array}$ & $\begin{array}{c}\text { TMX east } \\
\text { end cell }\end{array}$ \\
\hline Deuterium & 6 & 0.07 \\
Carbon & 1.5 & 0.05 \\
Nitrogen & 1.5 & 0.1 \\
Oxygen & 6 & 0.2 \\
Titanium & 10 & 0.1 \\
\hline
\end{tabular}


deuterium [22]), $n_{0}$ II is the density of $0 \mathrm{II}, n_{e}$ is the electron density, and $S_{0 I I}$ is the electron impact ionization rate out of 0 II (this determines the lifetime of 0 II in the plasma). Using the constant density model for $n_{0}$ II and assuming that $S_{0}$ II is a slow function of temperature:

$$
f_{0} \sim \frac{n_{0 \text { II }} S_{0 \text { II }} \int n_{e} d V}{I_{b}\left[1-\exp \left(-\frac{\sigma}{1.4 T}\right)\right] \int n_{e} d l}
$$

Substituting representative values $\left(n_{0}\right.$ II $=6 \times 10^{9} \mathrm{~cm}^{-3}, S_{0 \text { II }}=5 \times 10^{-9} \mathrm{~cm}^{3} / \mathrm{s}$, $\mathrm{n}_{\mathrm{eo}}=1 \times 10^{13} \mathrm{~cm}^{-3}, \mathrm{I}_{\mathrm{b}}=100 \mathrm{~A}, \int \mathrm{n}_{\mathrm{e}} \mathrm{dl}=3 \times 10^{14} \mathrm{~cm}^{-2}$ ) obtains $\mathrm{f}_{0}=0.03$ which agrees well with the value of 0.02 obtained for $2 X I I B$. According to the model, then, a significant source of oxygen in the TMX end cells is the neutral beam injectors.

Finally, there is evidence that the walls in the end cells are not a major source of oxygen. On Alcator A at MIT, it was noted that the oxygen influx dropped a factor of 5 as the walls were cooled to liquid nitrogen temperatures [34]. In the TMX end cells, there are many surfaces which can be cooled to liquid nitrogen temperatures in order to aid in vacuum pumping [35]. No correlation was observed, however, between the temperature of these surfaces and the oxygen emissions, even though the ambient pressure often dropped as much as a factor of 3 .

Nitrogen also has a significant concentration (nearly $1 \%$ ) in the TMX end cells. A spectral scan of N II 917 A (this is a multiplet so the energy is obtained using the method in Section III.5.2) indicates an ion energy of about $25 \mathrm{keV}$ which would suggest a high energy source such as the neutral beams. However, a single piece of evidence with large uncertainties is considered insufficient to make a definitive statement regarding the source of nitrogen.

Titanium was observed to be injected by the start-up plasma guns.

Figure V.8 shows the brightness of Ti IV $779 \AA$ when only the start-up guns are fired into the magnetic field. The emissions from both guns can be seen approximately $7 \mathrm{~cm}$ off axis. (The start-up guns operate by arcing across a stack of deuterium-loaded titanium washers. Therefore, it could be expected that some titanium would be injected with the deuterium.) Titanium could also be introduced from the walls and magnet cases. Titanium gettering is used to enhance the vacuum pumping so that, before nearly every shot, a fresh layer of titanium coats many surfaces in contact with the plasma. Sputtering or arcing could remove titanium from these surfaces and allow them to enter the plasma. 


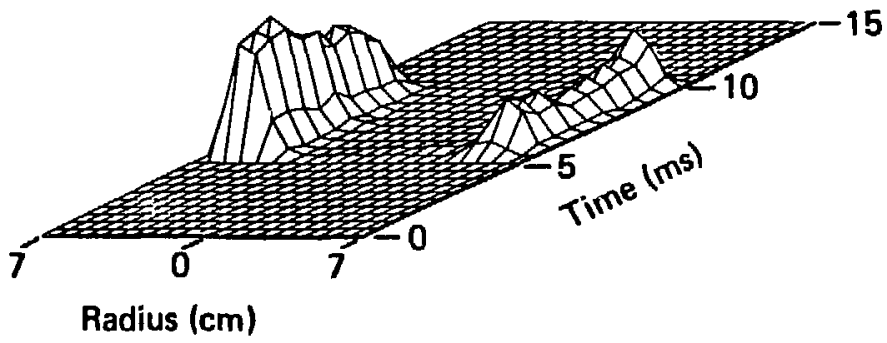

Fig. V.8. Brightness profile of Ti IV $779 \AA$ in the east end cell when only the start-up plasma guns are fired. This indicates that the start-up guns are a source of titarium in the end cells. 
This is a source of low energy titanium, so that this class of impurity should be poorly confined.

No source has been identified for carbon in the TMX end cell. A spectral scan of C IV 1548 \& indicates an ion energy of about $3 \mathrm{keV}$. This is too low an energy to be explained by a neutral beam source, unless the carbon were injected as part of a heavy molecule such as $\mathrm{CO}_{2}$. Methane $\left(\mathrm{CH}_{4}\right)$ is observed regularly with the resitual gas analyzer on TMX. Methane is believed to be produced from carbon present in the titanium getter wires; this would provide a source of low energy carbon. Other possible sources of carbon (for 2XIIB, but would also apply to the TMX end cells) are discussed at length in [16] and so are not discussed further here.

The central cell is not expected to provide a source of impurities to the TMX end cells. Ion energies in the central cell are less than $200 \mathrm{eV}$; impurities with this low an energy would not be well confined in the end cells due to the rapid scattering rate of low-energy multiply-charged ions into the loss region of velocity space. Furthermore, multiply-charged impurity ions in the central cell should be well confined axially by the potential difference between the end cells and the central cell and would not penetrate to the end cells.

\section{V.5.2 Central Cell Sources}

In the central cell, the condition of the vacuum chamber walls is observed to influence the emissions from carbon and oxygen. The brightnesses of singly- and doubly-ionized carbon and oxygen decreased as much as $70 \%$ after titanium gettering was performed on a previously ungettered section of the central cell wall. No changes occurred in other plasma parameters which might explain the decrease. An overall "clean-up" of 0 II has been observed at the beginning ci several runs. The brightness decreases with shot number as more gettering conditioned the vacuum chamber walls. Especially striking is a case when a vacuum break occurred before the run and loaded the walls with oxygen. The 0 II brightness decreased nearly an order of magnitude in less than 20 TMX shots. Nitrogen, however, showed no correlation with gettering in the central cell.

The end cells are not expected to be a major source of impurities in the central cell. High energy impurities are expected to be well trapped by the magnetic mirrors in the end cells. Low energy impurities in the end celis 
should preferentially be ejected to the end walls since the potential drop is significantly greater to the end walls $(\sim 1000-1500 \mathrm{~V})$ than to the central cell $(\sim 100-300 \mathrm{~V})$. 
VI. CONFINEMENT TIMES OF INTRINSIC IMPURITIES IN THE CENTRAL CELL OF TMX

\section{VI.1. INTRODUCTION}

The total confinement time in the central cell of TMX is a combination of axial and radial confinement time. Axial confinement times are determined by the increasing magnetic field strength and positive electrostatic potential at each end of the central cell. Radial confinement times are determined by cross-field transport rates which depend upon radial temperature, density and potential gradients along with non-adiabatic properties of the magnetic field [2]. The next section develops an expression for axial confinement times for ions of arbitrary mass and charge. The third section estimates the total confinement times of 0 VI ions in the central cell and the fourth section shows the effect of a finite confinement time on the charge state distribution of oxygen. Finally, Section 5 presents the results of a computer code which predicts oxygen brightness radial profiles by balancing ionization and recombination with a confinement loss term.

\section{VI.2. PREDICTED AXIAL CONF INEMENT TIME OF IMPURITIES}

The derivation of the expressions describing the axial confinement time of deuterium is thoroughly discussed elsewhere $[4,36]$ and may be generalized for the case of impurities. In the TMX central cell, the plasma densities were such that reither a high density nor low density approximation of particle scattering and loss was sufficient to describe the observed axial confinement times. For the low density limit (long mean free path), the loss rate is determined by how rapidly the ions scatter into the loss cone, at. which time they are assumed to be lost immediately. Therefore, the loss cone is empty and the confinement time is given by the product of the ion-ion scattering time $\tau_{i j}$ and the logarithm of the end cell-to-central cell mirror ratio $R$. In the high density limit (short mean free path), collisions are so rapid that particles remain confined, even in the loss cone, and the particle velocity distribution is Maxwellian everywhere. In this case, the loss cone is filled and the particle confinement time is determined by the plasma flow time $\tau_{f l o w}$ out both ends (add a factor of 2) of a magnetic mirror with ratio R. Monte Carlo calculations [36] predict that the total confinement is appropriately described by the sum of these two terms ( $r$ ather 
than the inverse of the sum of the inverses). The mirror confinement time $\tau_{0}$ of central cell ions with no electrostatic enhancement is:

$$
\tau_{0}=\tau_{i j} \log R+2 \tau_{f l o w} R
$$

The existence of an electrostatic potential at each end of the central cell enhances the confinement time due to the potential difference $\phi_{c}$ between the end cells and the central cell and the temperature of the central cell ions $T_{c}$ :

$$
\tau_{\phi}=\left[\tau_{i j} g(R) \frac{e \phi_{c}}{T_{c}}+2 \frac{R}{2} \tau_{f l o w}\right] \exp \left(Z_{i} e \phi_{c} / T_{c}\right)
$$

where

$$
e_{c}=T_{e p} \ln \left(\frac{n_{p}}{n_{c}}\right)
$$

and $g(R)$ is a slowly varying function of the mirror ratio $[g(10) \sim 4]$. The ion-ion collision time is given by [37]

$$
\tau_{i c}=\frac{1}{1.8 \times 10^{-19}} \frac{\left(A_{i} T_{c}+A_{c} T_{i}\right)^{3 / 2} m_{p}^{1 / 2}}{\left(A_{i} A_{c}\right)^{1 / 2} z_{i}^{2} z_{c}^{2} n_{c} \ln \Lambda_{c}}
$$

where the subscript $c$ refers to the central cell deuterium and $i$ refers to the impurity ions, $A$ is the atomic mass, $m_{p}$ is the proton mass, $Z$ is the charge, $n$ is the density, and $\ln \Lambda$ is the Coulomb logarithm ( $\ln \Lambda \sim 15)$. The ion-deuteron collision times are used here because self-scattering times among the impurity ions are much slower due to the low impurity densities. The flow time is determined by the length $L_{c}$ of the central cell and the flow velocity $v_{\text {f low }}$ :

$$
\tau_{f \text { low }}=\frac{L_{c}}{v_{f l o w}}=L_{c}\left(\pi A_{i} m_{p} / 8 T_{c}\right)^{1 / 2}
$$

The final expression for the axial confinement time of ions in the central cell of TMX is obtained by substituting Eqs. (VI.3), (VI.4), (VI.5) into (VI.2): 


$$
\begin{aligned}
\tau_{\phi} & =\left[1.4 \times 10^{6} \frac{\left(A_{i}+A_{c}\right)^{3 / 2}}{\left(A_{i} A_{c}\right)^{1 / 2}} \frac{T_{c}^{3 / 2}}{z_{i}^{2} n_{c}} \ln \left(\frac{n_{p}}{n_{c}}\right)\right. \\
& \left.+6.4 \times 10^{-7} \frac{L_{c} A_{j}^{1 / 2} R}{T_{c}^{1 / 2}}\right]\left(\frac{n_{p}}{n_{c}}\right)^{Z_{i}}
\end{aligned}
$$

assuming that $T_{i}=T_{c}$. Note that an enhanced confinement time requires that the end cell density be greater than the central cell density $\left(n_{p}>n_{c}\right)$. Table VI.l gives the predicted axial confinement times for the oxygen ionization states according to the above formalism for typical plasma parameters during TMX operation. The enhanced confinement times are longer than the TMX shot duration (about $25 \mathrm{~ms}$ ) for $Z>2$. This predicts that impurities are well confined axially in the central cell of TMX.

\section{VI.3 EXPERIMENTAL 0 VI CONFINEMENT TIME}

A method of estimating the total confinement time of 0 VI ions uses the density ratio of $0 V$ to $O V I$ and the rate equation for the 0 VI density which balances ionization and recombination with a confinement loss term:

$$
\frac{d n_{6}}{d t}=-n_{6} n_{e} s_{6}+n_{5} n_{e} s_{5}-n_{6} n_{e} \alpha_{6}+n_{7} n_{e} \alpha_{7}-\frac{n_{6}}{\tau_{6}}
$$

where $n_{5}, n_{6}, n_{7}$ are the density of $0 \mathrm{~V}, 0 \mathrm{VI}, 0 \mathrm{VII}, n_{e}$ is the electron density, $s_{i}$ is the ionization rate from the $i^{\text {th }}$ state, $\alpha_{i}$ is the recombination from the $i^{\text {th }}$ state, and $\tau_{6}$ is the conf inement time of $0 \mathrm{VI}$; in steady state, $\mathrm{dn}_{6} / \mathrm{dt}=0$. Even though 0 VII (helium-like oxygen) was not measured on TMX, the measured relative abundances of the observed ionization states (Table V.4) indicate that the density of 0 VII was quite low; this means that $n_{7} \sim 0$ in Eq. (V.9). Solving for $\tau_{6}$ from the remaining terms yields

$$
\tau_{6}=\left[n_{e}\left(R S_{5}-S_{6}-\alpha_{6}\right)\right]^{-1}
$$

where $R=n_{5} / n_{6}$. The family of curves in Fig. VI.l are calculated from Eq. (VI.8) by choosing different values of $R$. The points are ratios obtained by inverting the $0 V$ and $0 V I$ brightness profiles from various days of running and correspond to the on-axis densities $(r=0)$. The points are positioned along 
Table VI.l. Calculated central cell axial magnetic confinement times $\left(\tau_{0}\right)$ and enhanced electrostatic conf inement times $\left(\tau_{\phi}\right)$ for the ionization states of oxygen observed in TMX. This assumes $n_{p} / n_{c}=3, T_{e}=T_{c}=75 \mathrm{eV}, R=20, n_{c}=\times 10^{12} \mathrm{~cm}^{-3}$, $g(20)=4$.

\begin{tabular}{lllr}
\hline Species & Z & $\tau_{0}(\mathrm{~ms})$ & $\tau_{\phi}(\mathrm{ms})$ \\
\hline O II & 1 & 4.8 & 17 \\
0 II I & 2 & 4.0 & 25 \\
O IV & 3 & 3.8 & 61 \\
O V & 4 & 3.8 & 170 \\
O VI & 5 & 3.7 & 485 \\
\hline
\end{tabular}




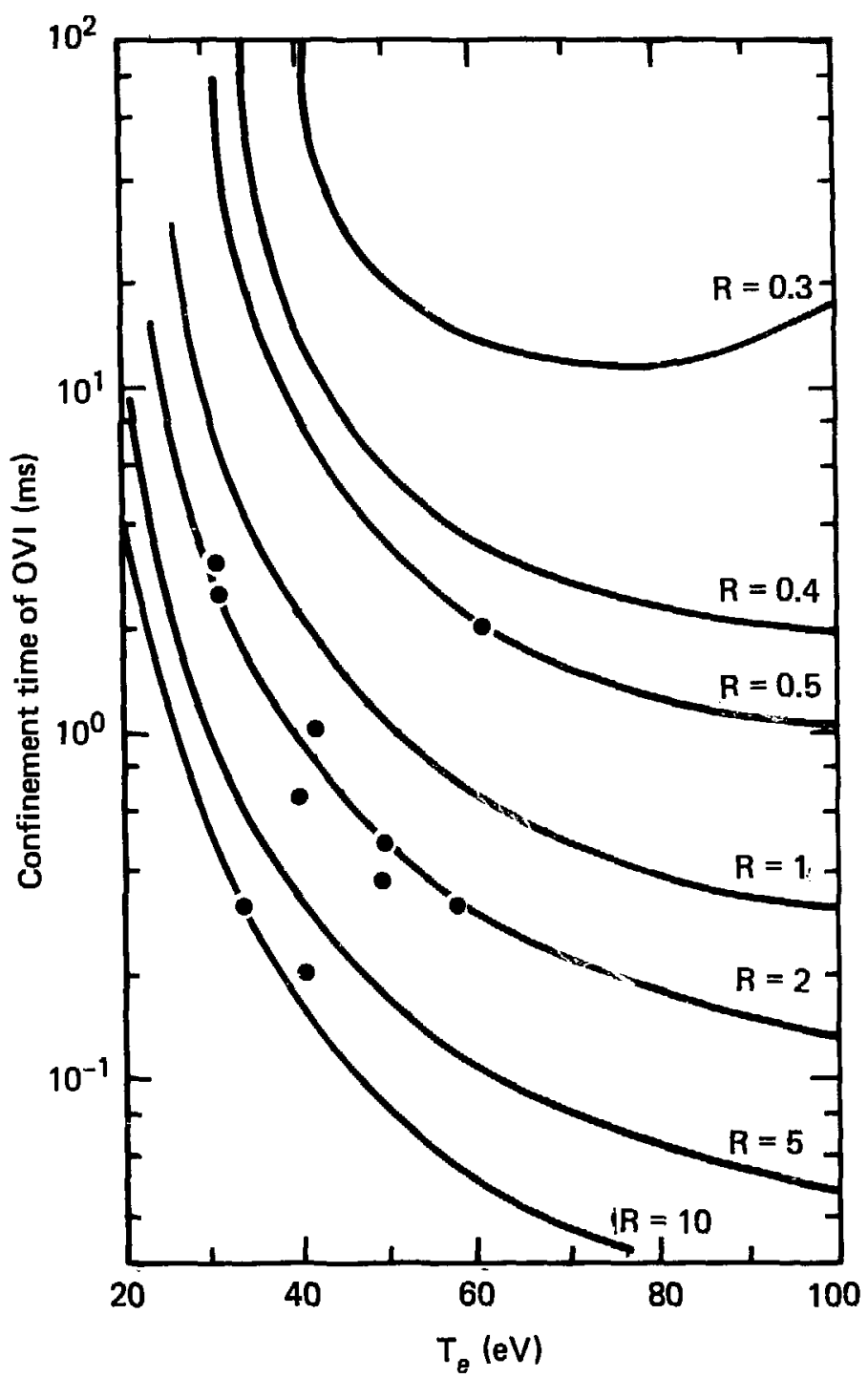

Fig. VI.I. Predicted confinement time of 0 VI vs electron temperature and density ratio of $0 \mathrm{~V}$ to $0 \mathrm{VI}$. Experimentally obtained values indicate a range in the 0 VI confinement time of 0.3 to $3 \mathrm{~ms}$. 
the appropriate curve by knowing the electron temperature measured on those TMX shots; the corresponding confinement time is then read from the vertical axis. The points indicate a range of values from $0.3 \mathrm{~ms}$ to $3 \mathrm{~ms}$ for the confinement times of $0 \mathrm{VI}$. The scatter in the data indicates that this is an order of magnitude estimate. Nevertheless, this range of values is considerably less than the $500 \mathrm{~ms}$ axial confinement time predicted in the previous section. This indicates that, for $0 \mathrm{VI}$, radial losses of impurities dominate in the central cell of TMX.

\section{VI.4. CONFINEMENT MODIFIED CORONAL EQUILIBRIUM}

The effect of a finite confinement time on coronal equilibrium may be calculated with a code which solves the set of simultaneous equations given in Eq. (VI.7). Assuming a constant-temperature, constant-density plasma in steady state, the code calculates the fractional abundance of each ionization state as a function of electron temperature. Figure VI.2(a) shows the results of the code for a very high (essentially infinite) confinement time. The results agree well with calculations which balance only ionization and recombination [23]. Setting the confinement time equal to $1 \mathrm{~ms}$ yields the results shown in Fig. VI.2(b). The effect of decreasing confinement time is to allow the lower ionization states to exist at higher temperatures; this decreases the average $Z$ at a given temperature. The same effect is predicted with an increasing high-energy neutral density [31] in which charge exchange recombination becomes important, such as in neutral beam heated devices (2XIIB, TMX end cells, TMX Upgrade, beam-heated tokamaks). On 2XIIB, in fact, the observed charge state distributions of oxygen were adequately modeled by balancing ionization with only charge exchange recombination [16].

This code also predicts thac 0 VII, which was not observed on TMX, makes up only about $6 \%$ of the total oxygen density at $50 \mathrm{eV}$ with a $1 \mathrm{~ms}$ confinement time. The error involved by assuming that $n_{7}=0$ in Eq. (VI.8) in the calculations of the 0 VI confinement time (Fig. VI.1) may be estimated by using the density ratio of 0 VII to 0 VI predicted by the code, which is 0.2 at $50 \mathrm{eV}$. Letting $n_{7}=0.2 n_{6}$ in Eq. (VI.8) and solving for $\tau_{6}$ results in only a $0.1 \%$ change in $\tau_{6}$ because $\alpha_{7}$ is a factor of 10 less than the other rates. 

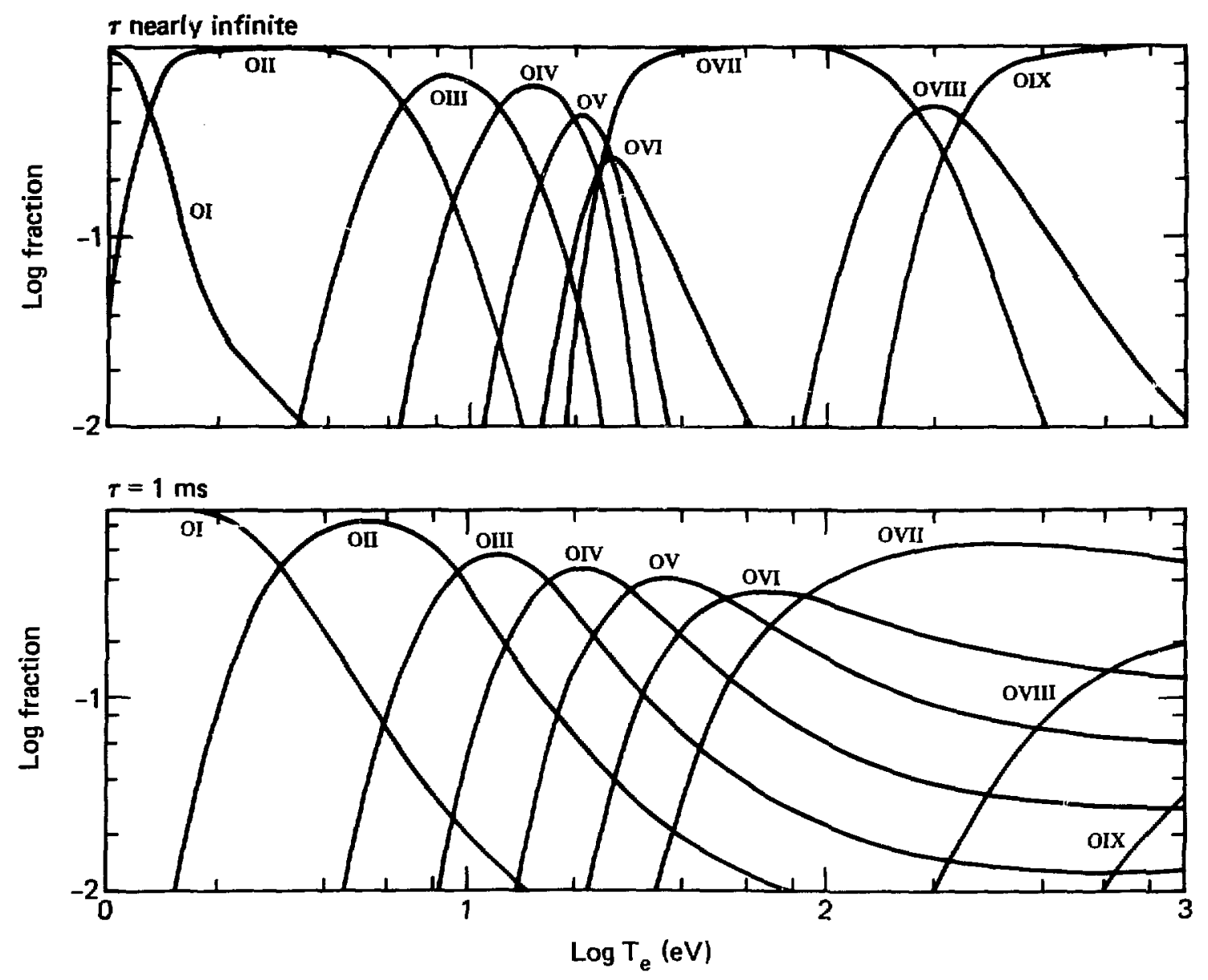

Fig. VI.2. Calculated oxygen ionization equilibrium for (a) nearly infinite confinement time and (b) 1 ms confinement time. 
VI.5. STEADY-STATE MODEL FOR OXYGEN IONS

\section{VI.5.1 Introduction}

This section describes a model which was used in an attempt to predict the steady-state radial brightness profiles of oxygen observed in the TMX central cell. In order to calculate the brightness profiles, the code requires radial profiles of electron density, electron temperature, neutral oxygen flux and oxygen ion confinement times as inputs. In the TMX central cell, only the electron density radial profile and the electron temperature on axis are measured. In particular, very little is known about the edge plasma conditions. The purpose of using a code such as this is to provide insight into those quantities which are not measured. Many attempts to match predicted profiles with observed profiles showed that a model which includes transport only through the mechanism of a cunfinement time is not adequate for impurities in the central cell plasma. Outward radial uransport of impurity ions in times which are short compared to recombination times is probably responsible for broadening the shapes of the observed radial profiles. This conclusion is consistent with the estimate of the impurity confinement times obtained in Section V.6.3. The model is desciibed in the following section; general trends are presented in Section VI.5.3 and the results are summarized in Section VI.5.4.

\section{VI.5.2 Description of Model}

This code calculates the density of each ionization state of oxygen by solving a set of nine coupled equations similar to Eq. (VI.7), one for each ionization state, which balance ionization and recombination with a confinement loss term. In steady state, the systeni of equations to solve is: 


$$
\begin{aligned}
& 0=-n_{1} n_{e} S_{1}+n_{2} n_{e} \alpha_{2}-n_{1} / \tau_{1}+\frac{2}{r_{0}} r_{1} \\
& 0=-n_{2} n_{e} S_{2}+n_{1} n_{e} s_{1}-n_{2} n_{e} \alpha_{2}+n_{3} n_{e} \alpha_{3}-n_{2} / \tau_{2} \\
& \dot{0}=-n_{i} n_{e} S_{i}+n_{i-1} n_{e} S_{i-1}-n_{i} n_{e} \alpha_{i}+n_{i+1} n_{e} \alpha_{i+1}-n_{i} / \tau_{i} \\
& \dot{0} \\
& 0=n_{8} n_{e} S_{8}-n_{9} n_{e} \alpha_{9}-n_{9} / \tau_{9}
\end{aligned}
$$

where $n_{i}$ is the density of the $i^{\text {th }}$ ionization state $\left(n_{1}\right.$ is the neutral oxygen density), $n_{e}$ is the electron density, $s_{j}$ is the electron impact excitation rate from the $i^{\text {th }}$ state, $\alpha_{i}$ is the recombination rate (including radiative and di-electroric recombination) from the $i^{\text {th }}$ state, $\tau_{j}$ is a general loss term describing the confinement time of the $i^{\text {th }}$ state, and $2 / r_{0} \Gamma_{i}$ is a source flux (normalized to the ratio of total surface area to total volume) which provides an initial density of neutral oxygen. The ionization and recombination rates used are given in Appendix A. These equations can be rearranged so that the density of each inization state is given in terms of the density of the state below:

$$
\begin{aligned}
& n_{1}=r_{1} / c_{1} \\
& n_{2}=n_{1} n_{e} s_{1} / c_{2} \\
& \vdots \\
& \dot{n}_{i}=n_{i-1} n_{e} s_{i-1} / c_{i} \\
& \vdots \\
& \dot{n}_{9}=n_{8} n_{e} s_{8} / c_{9}
\end{aligned}
$$


where

$$
\begin{aligned}
& c_{1}=n_{e} s_{1}-\frac{n_{e} s_{1} n_{e} \alpha_{2}}{c_{2}}+\frac{1}{\tau_{1}} \\
& c_{2}=n_{e} s_{2}+n_{e} \alpha_{2}-\frac{n_{e} s_{2} n_{e} \alpha_{3}}{c_{3}}+\frac{1}{\tau_{2}} \\
& \vdots \\
& c_{i}=n_{e} s_{i}+n_{e} \alpha_{i}-\frac{n_{e} s_{j} n_{e} \alpha_{i+1}}{c_{i+1}}+\frac{1}{\tau_{i}} \\
& \dot{c_{9}}=n_{e} \alpha_{9}+\frac{1}{\tau_{9}}
\end{aligned}
$$

Note that $c_{j}$ is given in terms of $c_{j+1}$, so that $c_{9}$ must be calculated first, then $c_{8}$, etc. When all of the $c_{j}$ are calculated, then the densities may be calculated starting with $n_{1}$, then $n_{2}$, etc. This procedure must be performed at every radial position. The $S_{i}$ and $\alpha_{j}$ are functions of electrc. temperature, which is itself a function of radius. The electron density, neutral oxygen flux, and confinement times are functions of radius also.

The predicted oxygen ion density profiles are multiplied by the given electron density profile and calculated excitation rate profile to obtain the volume emission rate profiles. The corresponding brightness profiles are obtained by applying the inverse of the matrix inversion [15] technique to the volume emission rate profiles. The electron density radial profile and the on-axis value of the electron temperature are determined experimentally and so were fixed in the model. The electron temperature radial profile, neutral oxygen flux and confinement times were varied in order to match the predicted brightness profiles with observed profiles.

\section{VI.5.3 General Trends}

The brightnesses of all the ionization states scale linearly with $\Gamma_{1}$, as one. d expect from Eqs. (VI.9). Obtaining the proper brightness ratios among the ionization states can, in general, be achieved in two ways: 
(1) adjusting the electron temperature, and (2) adjusting the confinement time as noted in Section VI.3. Thomson scattering measurements set the electron temperature on axis in the central cell, therefore that value is kept fixed in the model. Only the confinement time is varied in order to adjust the ratios of the ionization states on axis. The high ionization states respond most strongly to changes in confinement time. The reason for this can be seen by comparing the ionization and recombination times of, say, 0 II and 0 VI with a confinement time of $1 \mathrm{~ms}$. If $\mathrm{n}_{\mathrm{e}}=3 \times 10^{12} \mathrm{~cm}^{-3}$ and $T_{e}=50 \mathrm{eV}$, the ionization times of $0 \mathrm{II}$ and $0 \mathrm{VI}$ are $30 \mu \mathrm{s}$ and $3 \mathrm{~ms}$, respectively, and the recombination times are $30 \mathrm{~ms}$ and $300 \mathrm{~ms}$, respectively. Both species are dominated by ionization, but the ionization time of 0 II is much faster than any reasonable confinement time, while that of $0 \mathrm{VI}$ is comparable to the $1 \mathrm{~ms}$ confinement time determined in Section VI.3. Therefore, at $50 \mathrm{eV}$ the relative densities of the higher ionization states are determined mainly by confinement time while those of the lower ionization states are determined mainly by ionization $t$ imes.

The radial profile of each ionization state could be adjusted by changing the radial profiles of the neutral oxygen influx, electron temperature and confinement time. As mentioned before, the densities vary linearly with $\Gamma_{1}$ and the lower ionization states are insensitive to changes in confinement time. On the other hand, the ionization rates of all species become very sensitive to electron temperature below $20 \mathrm{eV}$, which is the range of temperatures expected near the plasma edge. Therefore, the radial profiles depend most strongly upon the shape of the electron temperature radial profile.

\section{VI.5.4 Discussion of Results}

This steady-state code which balances ionization and recombination with a confinement loss term appears to be unable to satisfactorily predict the observed oxygen brightness profiles. In particular, the radial extent of the higher ionization states is observed to be broader than predicted unless an e lectron temperature considerably higher than the 10-15 eV determined in Section V.3.1.4 is assumed at the plasma edge.

During puffer fueling, the brightness profile of $0 V I$ is just as flat and broad as the 0 II profile (Fig. V.4); it is unusual to have these two ionization states existing in the same regions of plasma. The best fit to the $O$ II and $O$ VI brightness profiles is obtained if the plasma is assumed to have 
a constant temperature at all radii. If a cylindrical plasma has constant density and temperature, then the resulting brightness profiles are parabolic. In this case, the electron density profile was slightly hollow on axis so that the predicted brightness profiles were somewhat broader than parabolic.

The best $f$ it to the brightness profiles during gas box fueling (e.g., Fig. V.3) requires not only an unreasonably high edge electron temperature ( $>30 \mathrm{eV}$ ) but also an increased confinement time toward the plasma edge. The high edge temperature is required to suppress the edge 0 II brightness and to broaden the $0 \mathrm{VI}$ profile.

Using "reasonable" input parameters for gas box operation (Fig. VI.3) does not provide a good match with the observed brightness profiles. The electron density profile is measured to be Gaussian with a peak value of about $5 \times 10^{12} \mathrm{~cm}^{-3}$ and a width of $26 \mathrm{~cm}$. The electron temperature is measured to be $50 \mathrm{eV}$ on axis; the profile is assumed to be Gaussian with an edge value of $10 \mathrm{eV}$ as indicated by Section V.3.2.4. The confinement time is assumed to be Gaussian with a peak value of $1 \mathrm{~ms}$ and drops a factor of 4 to the edge. The flux of neutral oxygen $\Gamma_{1}$ is chosen to peak at the plasma edge and decrease a factor of 4 in a Gaussian manner toward the center. The resulting predicted brightness profiles are shown in Fig. VI.4. While the match to the observed 0 II and 0 IV brightness profiles is fairly close, the profiles predicted for the higher ionization states are too narrow. Furthermore, the predicted ratio of the central chord brightnesses of 0 VI to 0 IV is almost a factor of 10 higher than observed. Lowering the confinement time to $150 \mathrm{ws}$, which is below the expected range of values indicated by the experimental density ratios of OV to OVI in Section V.6.3, results in closer agreement to the observed brightness ratio but still does not allow agreement between the observed and predicted radial profiles. Therefore, using "reasonable" values for input parameters to this code results in predicted brightness profiles which disagree with observed profiles. Outward radial transport, which is not included in this code, could explain the discrepancy. The recombination times for the higher ionization states are long compared to a $1 \mathrm{~ms}$ confinement time, so that an ion moving radially outward would be excited and radiate many times before recombining at some large radius. This would result in broader radial profiles of high ionization states than would be predicted without radial transport. 

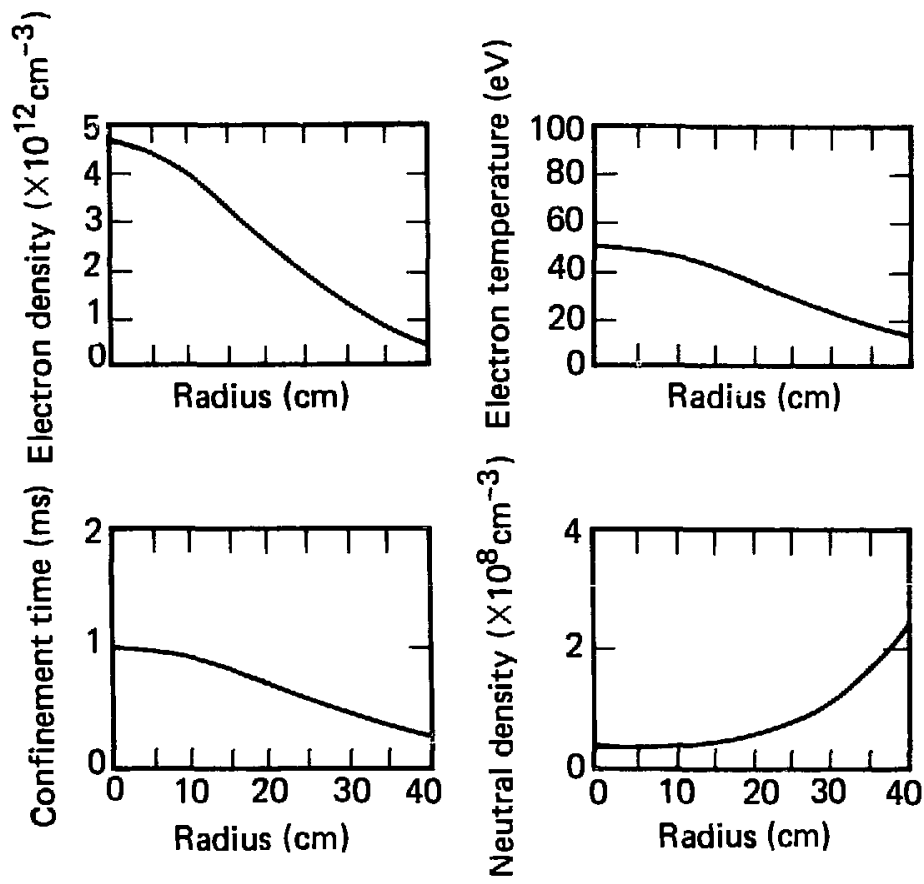

Fig. VI.3. "Reasonable" profiles of (a) electron density, (b) e lectron temperature, (c) confinement time, and (d) neutral oxygen density during gas box operation. 

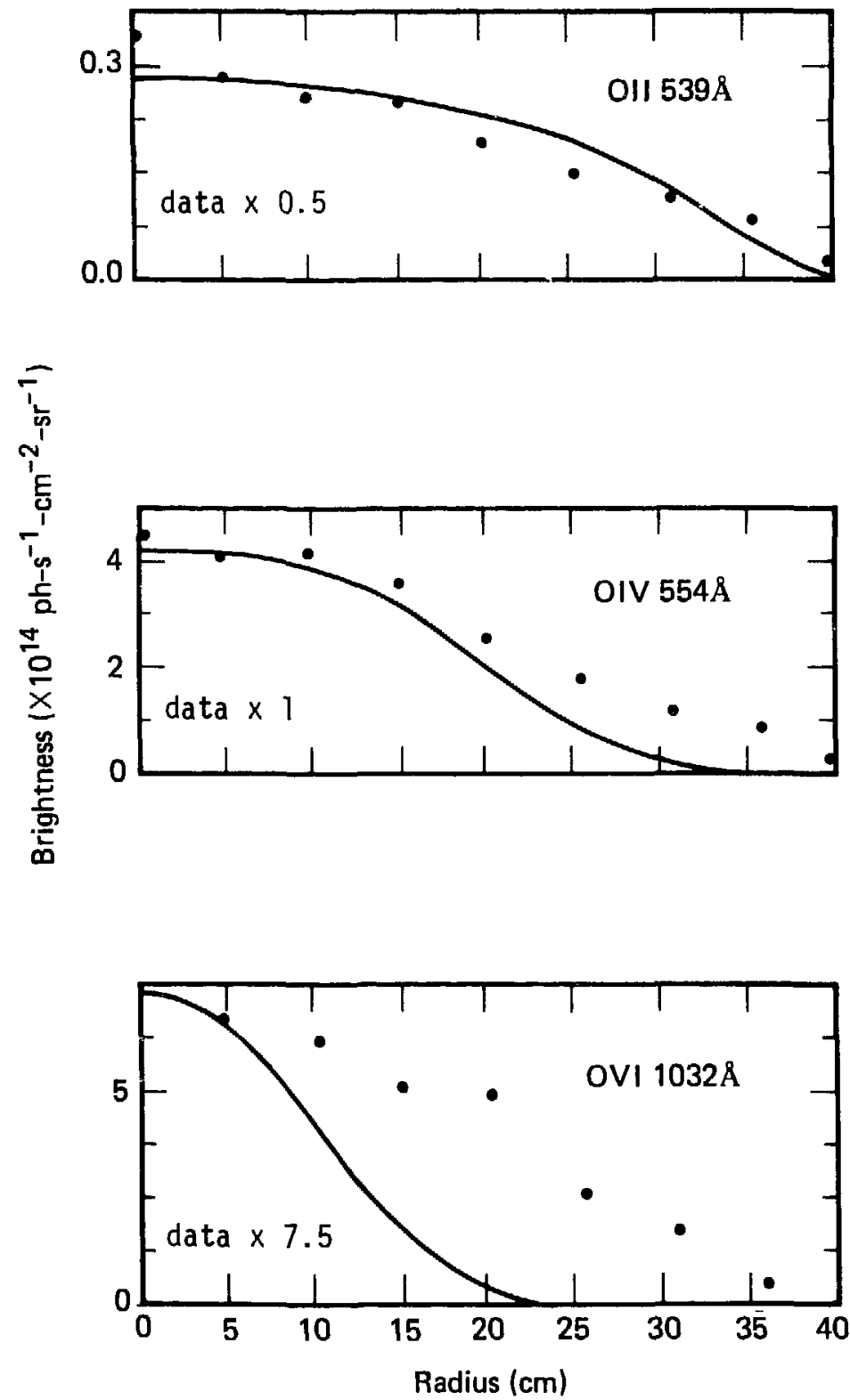

Fig. VI.4. Brightness profiles predicted by the model using "reasonable" input parameters during gas box operation. Actual brightness profiles are normalized to the code results for comparison. 


\section{IMPURITY PUFFING EXPERIMENTS IN THE CENTRAL CELL OF TMX}

\section{1. INTRODUCTION}

The previous chapters indicate that the intrinsic impurity concentrations are less than $0.5 \%$ for each species in the central cell even though the axial confinement times are predicted to be longer than the shot duration. Using the density ratios of $0 \mathrm{~V}$ to $0 \mathrm{VI}$ indicates that the total confinement time of $0 \mathrm{VI}$ is on the order of $1 \mathrm{~ms}$, which is comparable to the 3 to $5 \mathrm{~ms}$ confinement times determined for the deuterons. This suggests that radial losses must dominate in the central cell. However, the basic question of whether the low impurity concentrations in the central cell are due to the confinement time or to an unusually low source of impurities remains unanswered; a series of impurity injection experiments were proposed to address this issue. Oxygen and neon were puffed into the central cell chamber either before or during TMX shots to study the interaction of these gases with the plasma. The time behavior of the injected emissions show that (1) impurities injected during the shot accumulate in the outer region of the central cell plasma while only a small fraction penetrates into the central plasma region, (2) excess impurities injected before the shot actually leave the plasma during the shot, and (3) impurities injected into the central cell plasma are not observed in the end cell plasmas. These results provide support for the evidence presented in Chapter VI which indicates that impurities are transported radially outward in the central cell plasma. The next section explains the experimental procedure and data analysis. Sections 3 and 4 present the data and results.

\section{VII.2. EXPERIMENTAL PROCEDURE}

0xygen and neon gases were puffed from between two limiters into the central cell of TMX from the top of the tank at the machine midplane. A fast solenoid-driven value (TMX \#44) was connected to a bottle of high purity gas to provide the injected impurity. The amount injected was controlled by the pressure of gas determined by the gas-bottle regulator, a screw which set the orifice size between the valve and the TMX vacuum, and the length of the pulse which actuated the : alve (set at $5 \mathrm{~ms}$ in 311 cases for these experiments). Two different timing sequences were used according to the experiment 
performed: (1) the early puff, in which the valve was fired from $-4 \mathrm{~ms}$ to $+1 \mathrm{~ms}$ into the timing sequence, and (2) the iate puff, in which the valve was fired from $+12 \mathrm{~ms}$ to $+17 \mathrm{~ms}$ into the sequence. The early puff provided a source of constant impurity density before the TMX shot, while the late puff simulated the interaction of the edge plasma with an outside source of impurities such as the walls.

The amount of gas injected was determined by a compromise between obtaining reasonable signal levels above background and perturbing the plasma as little as possible. Generally, a factor of 2 to 5 increase in signal over background was considered sufficient for the low density ionization states; this resulted in a factor of 10 to 20 increase in signal for the high density states. Almost no change in plasma parameters was observed during these experiments. Only the heavy ion beam probe, which measures the plasma potential, showed a significant $(\sim 35 \%)$ increase in signal on some shots when the impurities were injected [38]. For the data considered here, however, none of the observed plasma parameters varied more than $20 \%$ due to the injected gases. Therefore, the plasma parameters are considered to be constant during these experiments.

The ernissions from the injected gases were monitored with the LLNL-SIDS which was mounted above the central cell midplane. Since the field of view of this instrument was $37 \mathrm{~cm}$, two radial fields of view were used to study half the piasma. This allowed some overlap in the two views while ensuring that both the edge and the central portions of the plasma were observed. In the case of oxygen, the amount injected had to be distinguished from the ambient levels. Therefore, an extra set of two shots, one for each field of view, was taken under identical conditions but without firing the puffer valve in order to establish the background signals. These background shots were then subtracted from the puffer shots in order to determine the time evolution of only the injected quantity. No background shots were required for neon since there was no neon in the plasma except when it was injected. Because such a large number of shots was required to perform this experiment, only the north half of the plasma was observed during the neon and oxygen injection.

Subtracting the brightness profile without the puff from the profile with the puff yields the brightness profile due to only the injected quantity. The (matrix) inversion of this profile is then divided by a constant excitation rate coefficient and the average electron density radial profile obtained from those shots in order to obtain the radial density profile. This procedure is 
performed at each of 80 timepoints during the shot. Since the inverted profiles tend to be somewhat noisy, some smoothing is generally applied to the final density matrices. Densities less than zero are made equal to zero and a 3-point sliding average in both radius and time is applied. Figures VII.1(a) and (b) show the difference between an unsmoothed and smoothed density profile.

\section{VII.3. LATE PUFFING EXPERIMENTS}

This section presents the data obtained from the late puffing experiments. The puffer valve was fired from 12 to $17 \mathrm{~ms}$ into the timing sequence, which corresponds to the steady-state portion of the TMX shot. Each injected impurity is discussed separately in the following sections.

\section{VII.3. 1 Late Puffing-0xygen}

Figure VII.1(b) shows the temporal and spatial profile of the total puffed oxygen density. The behavior of the total puffed density is determined by adding the contribution from each ionization state; the signal from the ambient oxygen has been subtracted out. It can be seen that most of the puffed density remains ac the outer edge while only a small amount penetrates to the central region of the plasma. Figure VII.2 shows the radial profile of the total puffed density at $25 \mathrm{~ms}$ into the timing sequence. The peak of the puffed oxygen density profile is at $38 \mathrm{~cm}$ where the limiter is located; at 30 $\mathrm{cm}$, the density is less by a factor of 3 and, at $10 \mathrm{~cm}$, the density is less by a factor of 15 from the peak value. This indicates that very little of the puffed density penetrates within $r<30 \mathrm{~cm}$. Calculating a volume average concentration for the two regions of $0<r \leq 30 \mathrm{~cm}$ and $30<r \leq 45 \mathrm{~cm}$ iridicates nearly a factor of 10 higher average concentration in the outer region (1.4\%) than in the central region (0.16\%). Once established, this density profile is maintained until the end of the shot. This is seen in Fig. VII. 3 which shows the temporal profiles of the puffed oxygen density at $38 \mathrm{~cm}, 30 \mathrm{~cm}$, and $20 \mathrm{~cm}$. (The fluctuations are due to a noisy background shot.) The densities at the inner radi i remain constant with time; the density at $38 \mathrm{~cm}$ appears to decrease with time in this case, but data taken on other days of singly-ionized oxygen, which is about $70 \%$ of the total oxygen density at the edge, indicate that the density remains constant with time at $38 \mathrm{~cm}$ also. Furthermore, it can be seen that the density 


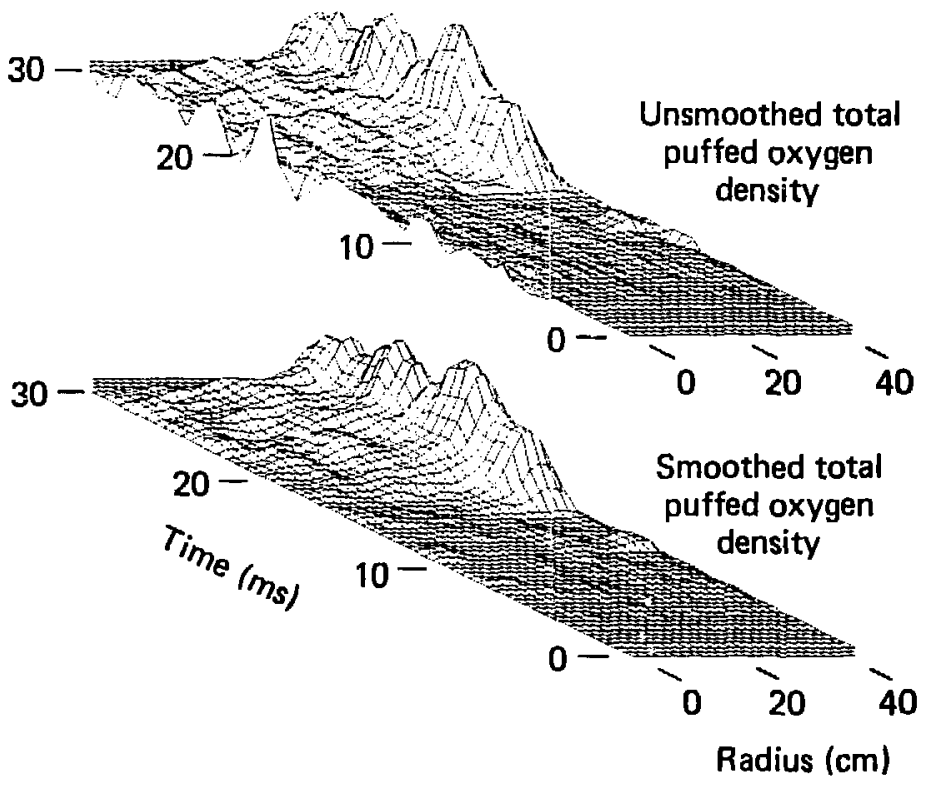

Fig. VII.1. (a) Unsmoothed and (b) smoothed radial and temporal profile of total puffed oxygen density in the central cell. 


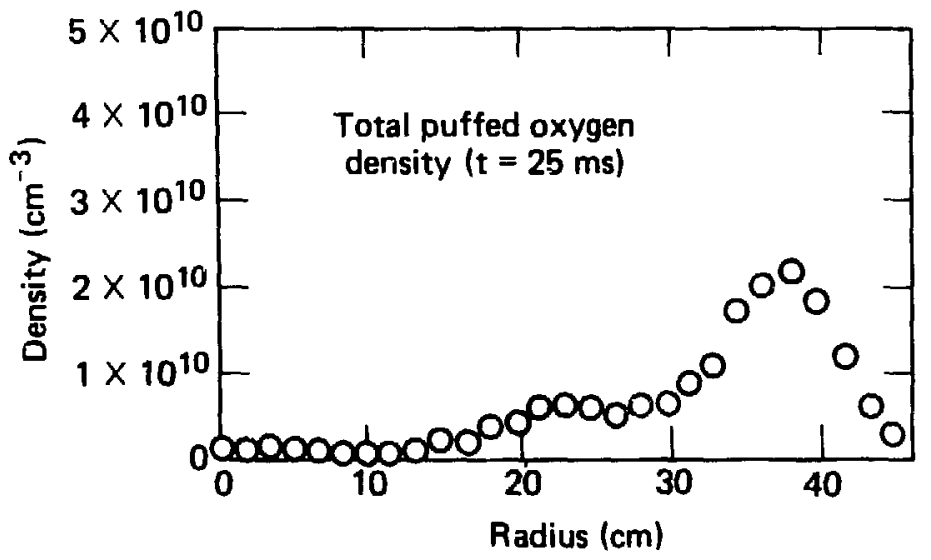

Fig. ViI.2. Radial profile of total puffed oxygen density in the central cell at $25 \mathrm{~ms}$ during the 1 ate puffing experiments.. 
TIME PROF ILES AT 20, 30, 38cm RADII

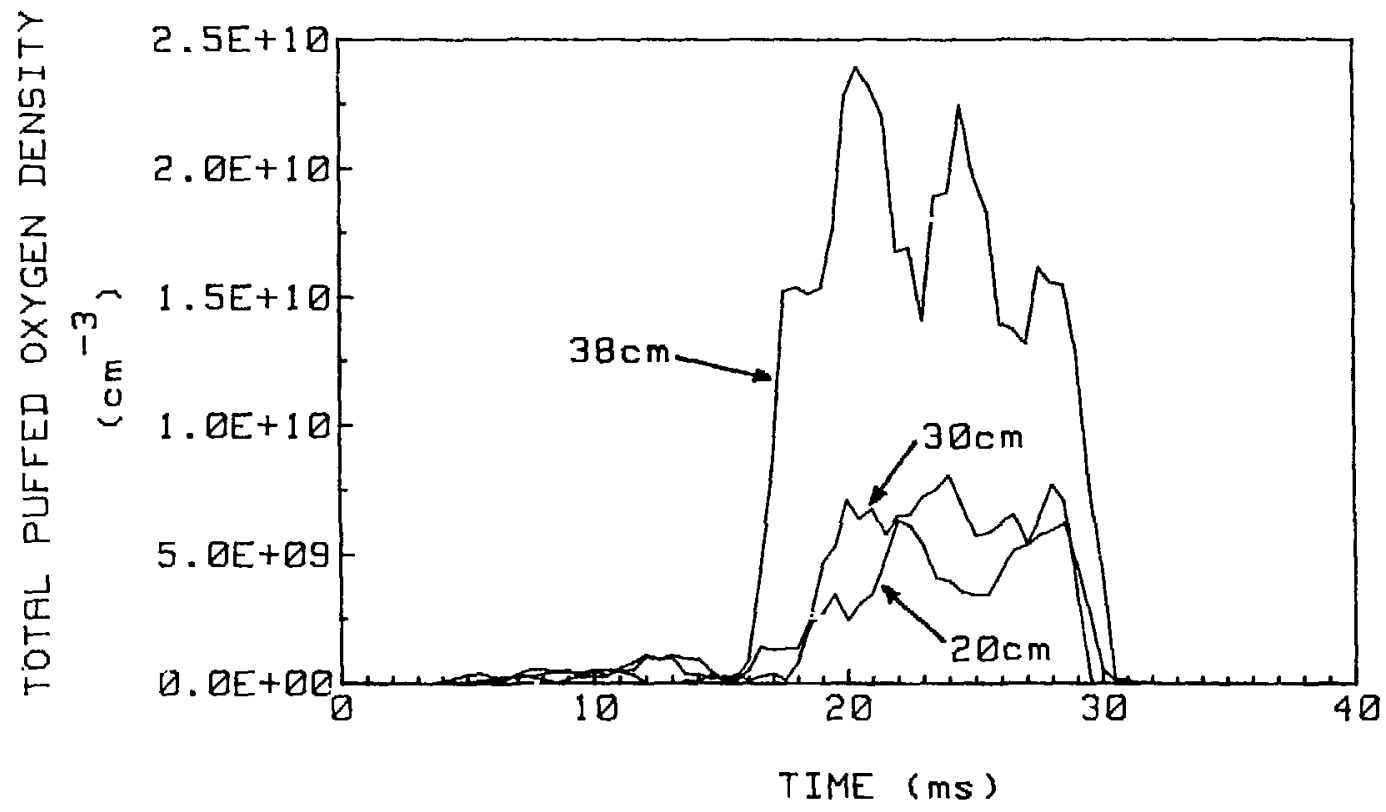

Fig. VII.3. Temporal profiles of total puffed oxygen derisity at $38 \mathrm{~cm}$, $30 \mathrm{~cm}, 20 \mathrm{~cm}$ during the late puffing experiments. 
rise occurs at later times for the inner radii. Plotting in Fig. VII.4 the times at which the density reaches $5 \times 10^{9} \mathrm{~cm}^{-3}$ at each radial position gives a measure of the rate at which the total density penetrates the plasma; the line drawn in Fig. VII.4 corresponds to a rate of $3 \mathrm{~cm} / \mathrm{ms}$. This penetration rate and the steady-state density gradient in Fig. VII.2 are the result of the competition between an inward force due to the oxygen density gradient and an outward force due to the plasma density, temperature, and electric potential gradients. Higher ionization states are presumably affected more strongly by the electric field, but differences in penetration rates of the various ionization states could not be seen in the data.

\section{VII.3.2 Late Puffing-Neon}

Neon was puffed into the central cell with the same timing sequence used for oxygen: 12 to $17 \mathrm{~ms}$. The reasons for puffing neon as well as oxygen are two-fold: (1) the ionization potential of neutral neon is $21.6 \mathrm{eV}$, versus $13.6 \mathrm{eV}$ for neutral oxygen, so that the neon should have penetrated the plasma more ceeply than oxygen before ionizing; (2) neon is not adsorbed onto titanium-gettered surfaces as efficiently as oxygen, so that the amount of recycling was expected to be higher in the case of neon.

The comparison between neon and oxygen is based upon comparing the singly-ionized neon and oxygen which were both observed during a single run. Figure VII.5 compares the radial profiles at $25 \mathrm{~ms}$ into the timing sequence; the two profiles are nearly identical. The ratios of the volume average concentration for $r<30 \mathrm{~cm}$ and $r>30 \mathrm{~cm}$ in the two cases are both about 7 . Therefore, no difference is seen in the data between the penetration of neutral neon and oxygen. The effect of the titanium gettering may be seen by comparing the observed Ne II and 0 II densities with the amount of each gas injected. The puffer valve was calibrated several days later in a separate vacuum system for the valve settings used during this experiment. The calibration tank was not gettered, while the TMX central cell was gettered during the experiment. The observed density of Ne II was over $2 \times 10^{10} \mathrm{~cm}^{-3}$ while that of 0 II was about $4 \times 10^{9} \mathrm{~cm}^{-3}$; the total number of neon atoms injected was about $3.5 \times 10^{19}$ atoms while about $6 \times 10^{19}$ oxygen atoms were injected (the volume of the central cell vacuum tank is $10^{7} \mathrm{~cm}^{3}$ ). The fractional amount of injected neon appearing as Ne II is approximately 10 times greater than the 


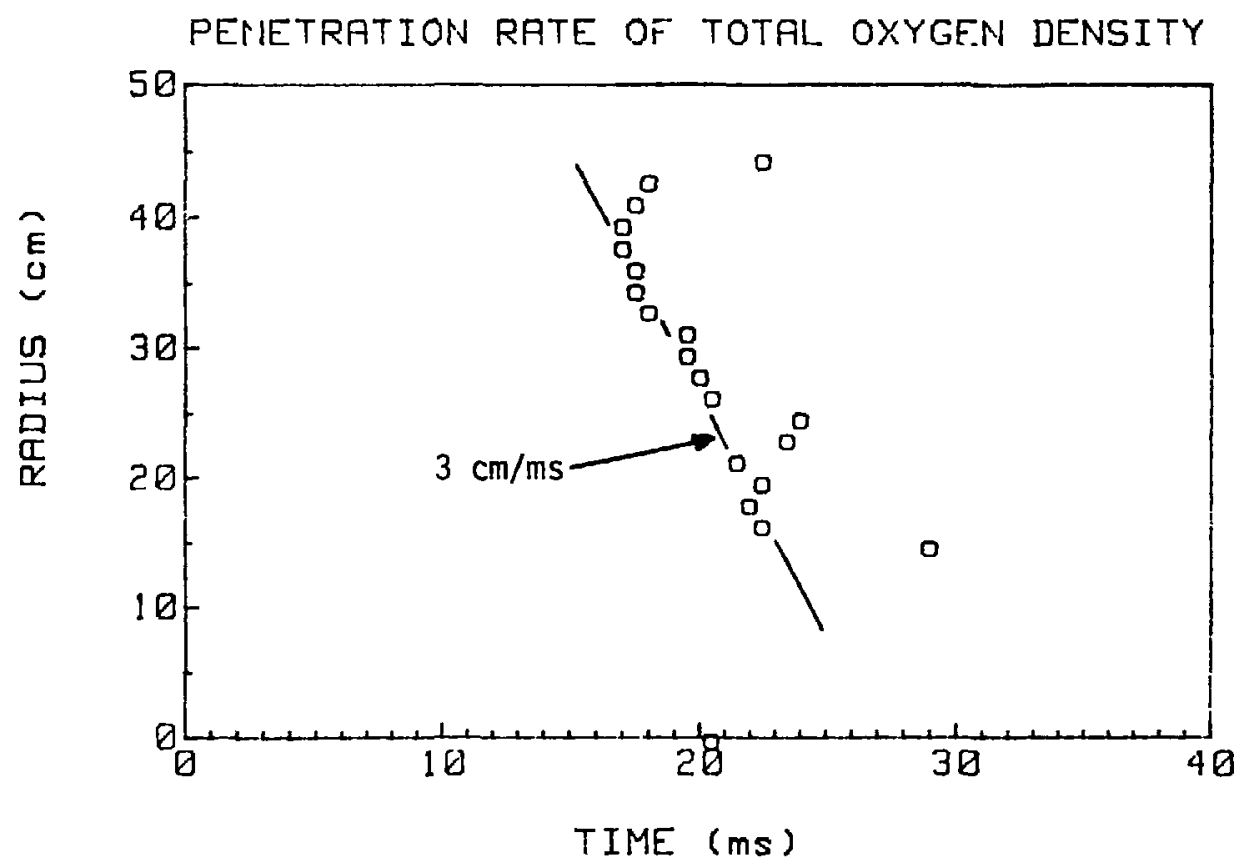

Fig. VII.4. Penetration rate of total puffed oxygen density in the central cell during the late puffing experiments. 


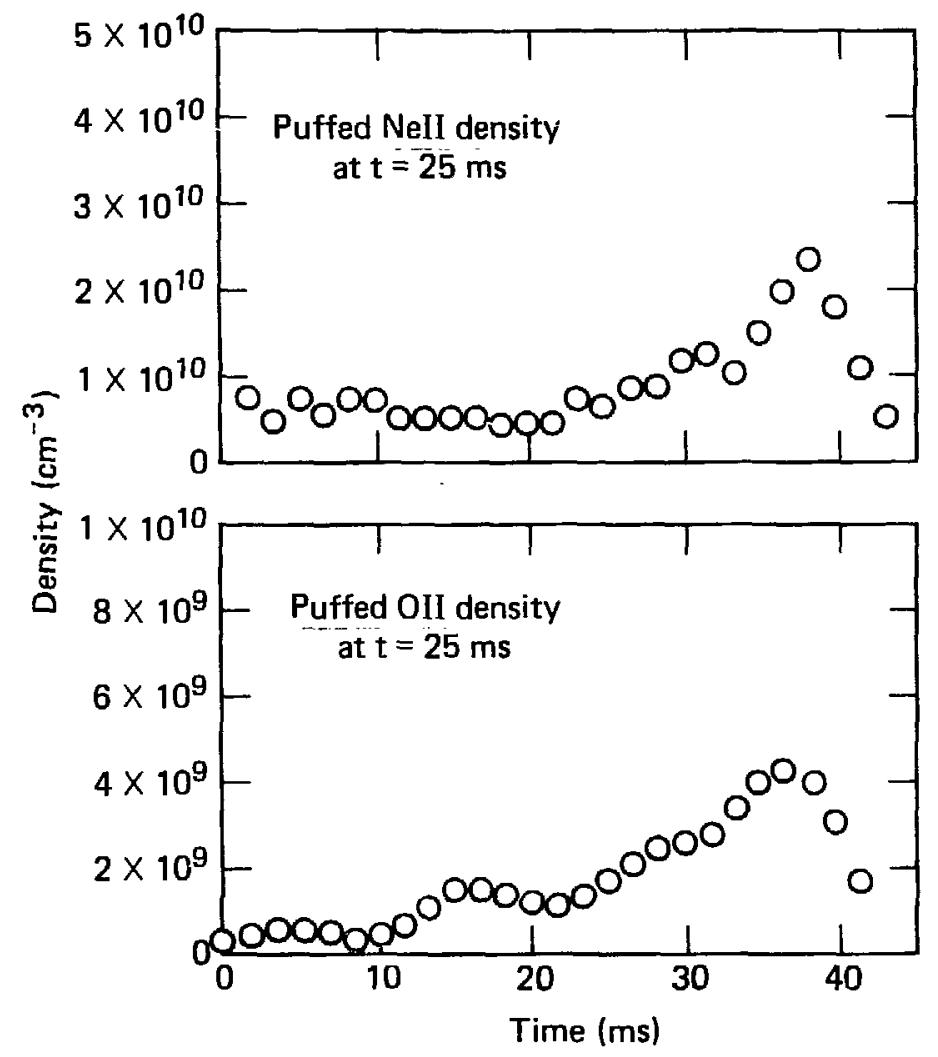

Fig. VII.5. Radial profile of puffed (a) Ne II and (b) 0 II density in the central cell at $25 \mathrm{~ms}$. 
amount of injected oxygen appearing as 0 II. The titanium gettering is significantly more effective at pumping oxygen than neon, as expected.

\section{VII.3.3. Late Puffing - End Cell Results}

Data was taken with the instruments on each end cell during some of the late puffing experiments. No change in signal was observed from either end cell plasma when the impurity gases were puffed into the central cell. Instrumental sensitivity puts an upper limit of 0.03 on the ratio of injected impurity in the end cells to that in the central cell. This result gives direct evidence that impurity ions are well confined axially in the central cell of TMX and supports the predictions of the calculations presented in Section VI.2.

\section{VII.4. EARLY PUFFING - OXYGEN}

Only oxygen was used for the early puffing experiment; the puffer valve was fired from -4 to $+1 \mathrm{~ms}$ into the timing sequence. The start-up guns fired at $+3 \mathrm{~ms}$, so that the puffed cloud of oxygen had at most $7 \mathrm{~ms}$ to expand into the vacuum chamber before the plasma shot occurred. Room temperature oxygen molecules travel about $27 \mathrm{~cm} / \mathrm{ms}$, so that they traveled almost $200 \mathrm{~cm}$ in $7 \mathrm{~ms}$. The distance from the valve at the machine midplane to each end of the central cell is $250 \mathrm{~cm}$; therefore, the oxygen just filled the central cell volume before the TMX shot.

Figure VII.6 shows the injected brightnesses (the background levels due to the ambient species are subtracted out) of 0 II, 0 IV, and 0 VI along the central chord. The brightnesses of all ionization states decrease with time; this decrease is observed at all radii which indicates that the excess puffed oxygen is leaving the plasma. However, the decay rates are not an indication of the confinement time of each state because recycling adds an unknown source term which increases the decay times. The high ionization states, though, would have the smallest source terms because the finite confinement time means that many ions are lost from the plasma before ionizing to the high ionization states. Figure VII.6 shows that the lower ionization states decay slowly while 0 VI decays in about $1.5 \mathrm{~ms}$. This decay time of 0 VI is on the order of the confinement times obtained for 0 VI in Section VI.3. Since D VI does not ionize further into 0 VII (Section V.6.3) and the recombination time from 0 VI 


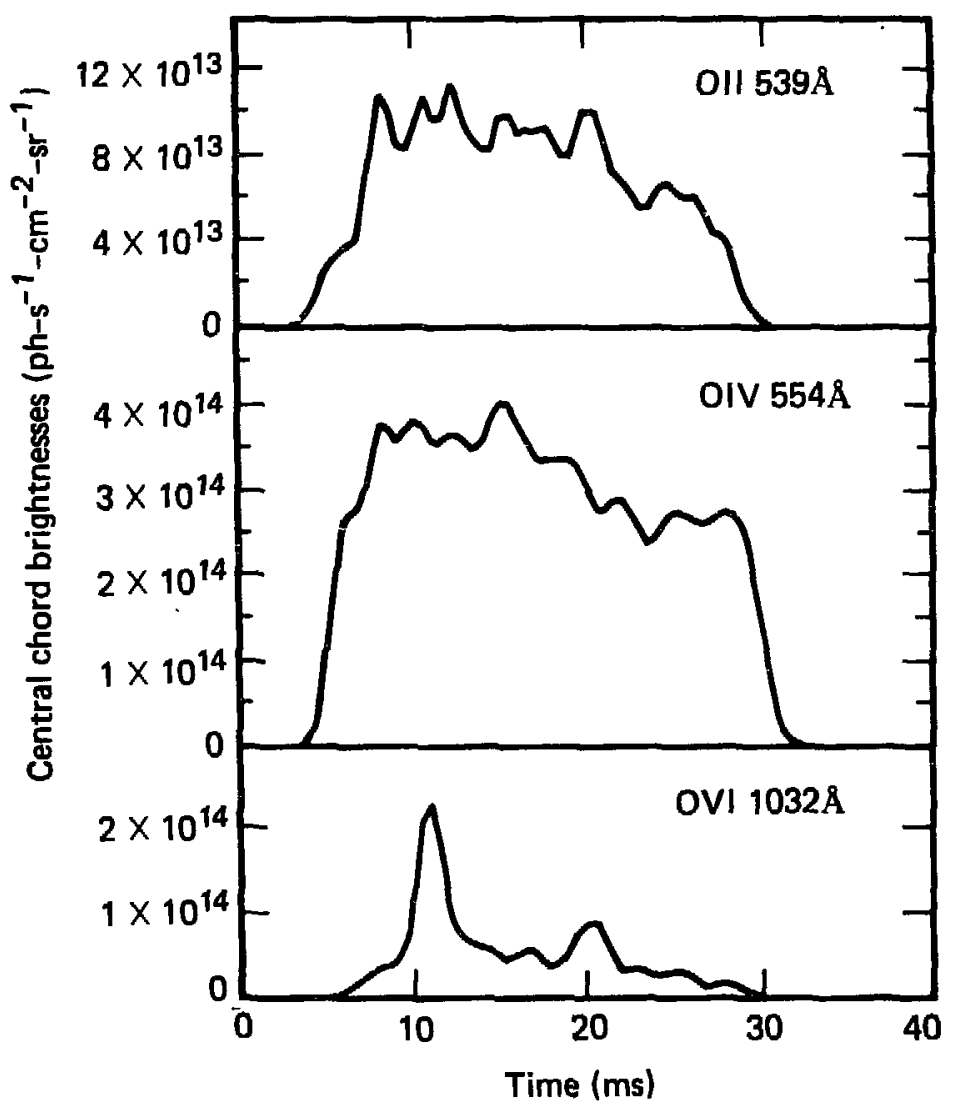

Fig. VII.6. Temporal profiles of injected 0 II, 0 IV, 0 VI central chord brightnesses during early puffing experiments. 
to $0 \mathrm{~V}$ is about $20 \mathrm{~ms}$ under these plasma conditions, the decay time of this peak must be due to a confinement loss of 0 VI. The peak in the 0 VI is observed at about $11 \mathrm{~ms}$ into the timing sequence, which is about $4 \mathrm{~ms}$ after the central cell plasma builds up to its steady-state density. This delay time agrees with the calculated time required $(\sim 3 \mathrm{~ms})$ to ionize from neutral oxygen to 0 VI at an electron density of $5 \times 10^{12} \mathrm{~cm}^{-3}$ and an electron temperature of $50 \mathrm{eV}$.

\section{VII.5. DISCUSSION}

The late puffing experiments indicate that the central cell plasma resists the radial penetration of ions into the central regiun of plasma. There appears to be a well-defined boundary at about $r=30 \mathrm{~cm}$ within which the injected species do not readily penetrate. This suggests that the main interaction of the central cell plasma with external influences, such as recycling from the walls, is limited to the outer layer of plasma. The exception to this is the plasma-wall interaction of the end fan regions near the gas boxes where the plasma is only $4 \mathrm{~cm}$ thick (Section II.3); neutral atoms can readily penetrate to the inner field lines in these regions before ionizing. This explains the broader (and even slightly hollow) plasma density profiles observed when the central cell plasma is fueled by the puffer method compared with the narrower profiles observed during gas box fueling. In principle, it should be possible (within limits) to create a desired plasma density radial profile--and, hence, a desired plasma potential radial profile--by choosing the appropriate combination of gas box and puffer fueling during a given TMX shot. The heavy ion beam probe did, in fact, measure a nearly constant radial plasma potential for $r<28 \mathrm{~cm}$ in the case of puffer fueling, while a parabolic profile was observed during gas box fueling [38]. An interesting experiment would have been to puff impurities into a puffer-fueled plasma to observe the effect on penetration of the different potential profiles. Furthermore, puffing impurities near the end fans or through the gas boxes would have indicated whether radial penetration of ions into the central region of plasma does occur at these locations.

The early puffing experiments show that impurities in the central cell are not well confined. That none of the injected impurities were observed in either end cell indicates that the impurity losses must be radial rather than axial. Furthermore, the decay time of the 0 VI brightness during the early 
$-100-$

puffing experiments is in good agreement with the range of O VI confinement times determined in Section VI.3. 


\section{A. 1 ELECTRON IMPACT EXCITATION RATES}

Two sources of excitation rates for the light impurities were used during this study. A LASL report [39] gives different empirical fits of theoretical computations for $\Delta n=0$ transitions. These fits are given for each transition in terms of the electron temperature, transition energy and a set of coefficients which must be looked up in an extensive set of tables.

For transitions not listed in the LASL report, values were obtained from $[40]$

$$
Q_{j}\left(T_{e}\right)=\frac{1.6 \times 10^{-5} f_{i k}\langle\bar{g}\rangle \exp \left(-E_{i} / T_{e}\right\rangle}{E_{i}\left(T_{e}\right)^{1 / 2}}
$$

where $f_{i k}$ is the oscillator strength [41] for the given transition, <g> is the average Gaunt factor (obtained from [42] for nitrogen and oxygen; the values for carbon were taken to be the same as those of the identical transitions in nitrogen), $E_{j}$ is the transition energy, and $T_{e}$ is the electron temperature. The average Gaunt factors and oscillator strengths used in Eq. (A.1) are listed in Table A.1. The resulting functions of electron temperature are plotted in figures A.1 - A.3. For comparison, the values obtained from the LASL report are plotted in figures A.4 - A.5. (The excitation rates for Lyman alpha in Fig. A.4 were obtained by numerical integration of the excitation cross-section [43] over a Maxwellian electron temperature distribution.) Be low $100 \mathrm{eV}$ (the range observed on TMX), the two sources agree within 50\%; above $100 \mathrm{eV}$, the LASL values are higher. The authors of the LASL report suggest that the Gaunt factor method should not be used unless no other source is available. Values for the excitation rates from the LASL report were used whenever possible in this study.

\section{A.2 ELECTRON IMPACT IONIZATION RATES}

The values used for electron impact ionization rates are those obtained from Lotz [44]; values for all ionization states of carbon, nitrogen, oxygen, and neon are presented in Figs. A.6 - A.8. Craridall [45] compares various expressions for ionization rates with crossed-beam experiments; Lotz is 


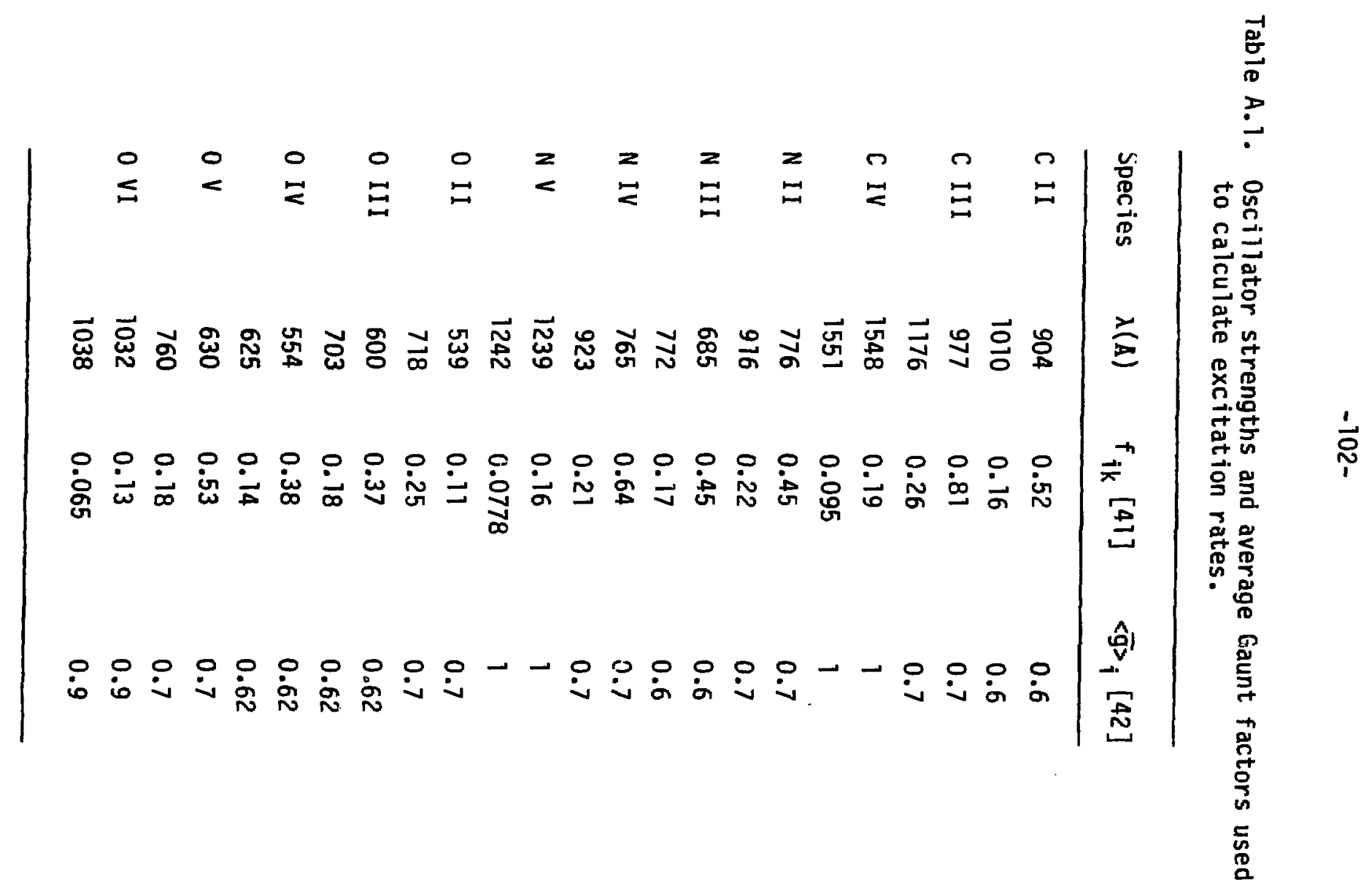


generally within a factor of two of the experimental results, especially at the low electron temperatures encountered on TMX.

\section{A.3 RADIATIVE AND DIELECTRONIC RECOMBINATION RATES}

The values used for recombination rates of oxygen were obtained from a code used by Terry [17]; they were modified slightly to obtain closer agreement with values presented by Mattioli [46] which include both radiative and di-electronic recombination. These results are plotted in Fig. A.9. 

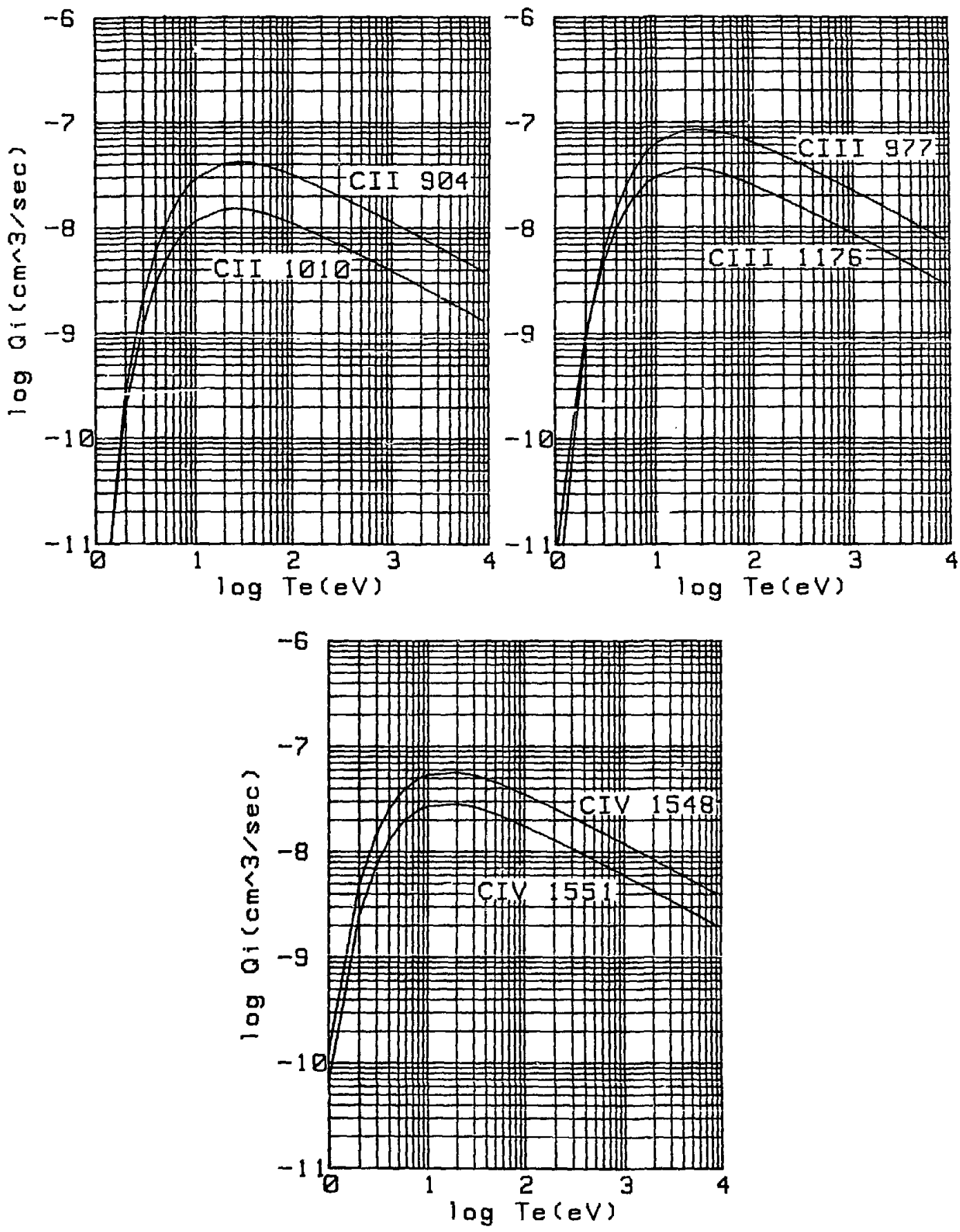

Fig. A.1. Excitation rates for transitions of carbon ions (Gaunt factors by Davis [42]). 

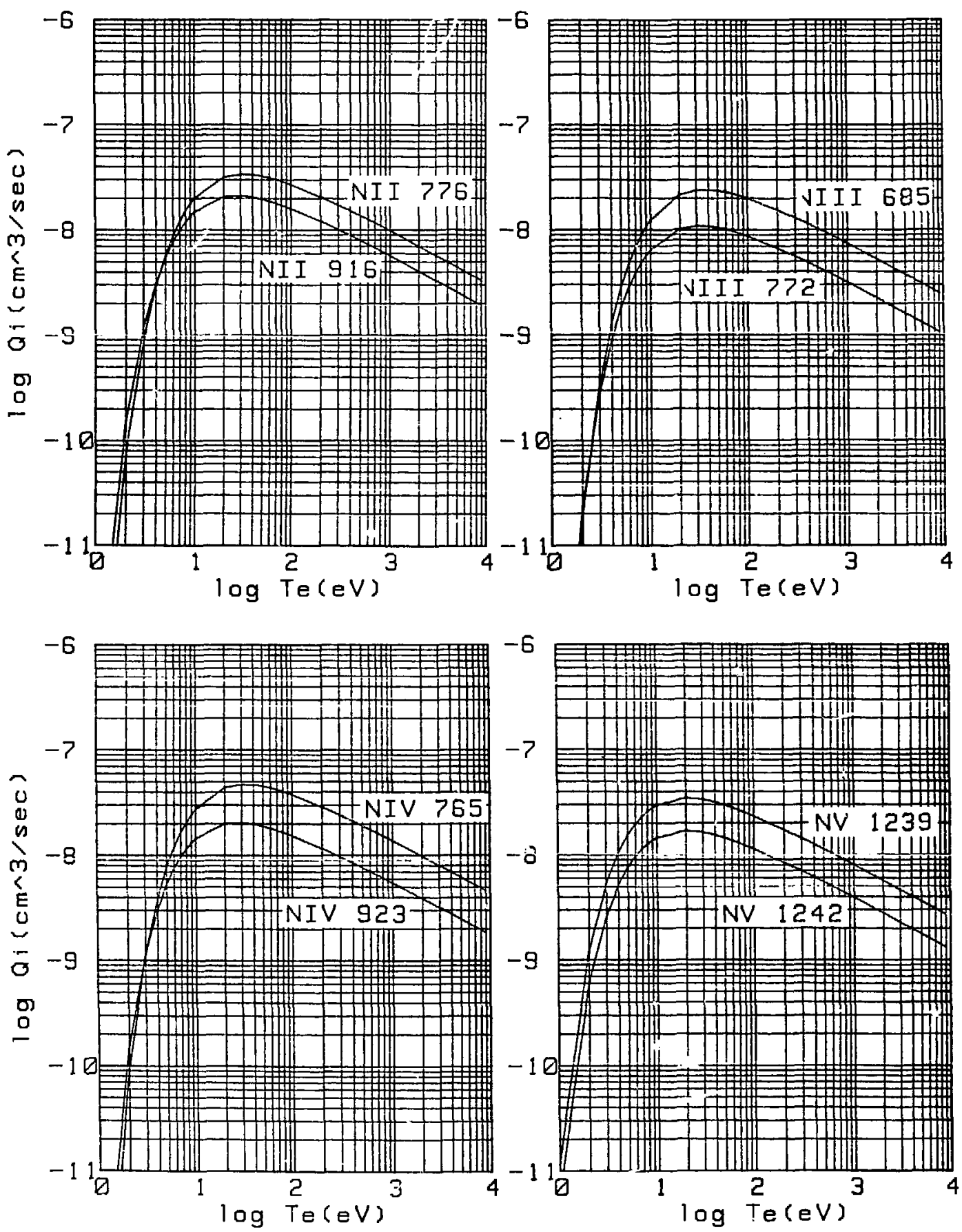

Fig. A.2. Excitation rates for transitions of nitrogen ions (Gaunt factors by Davis [42]). 

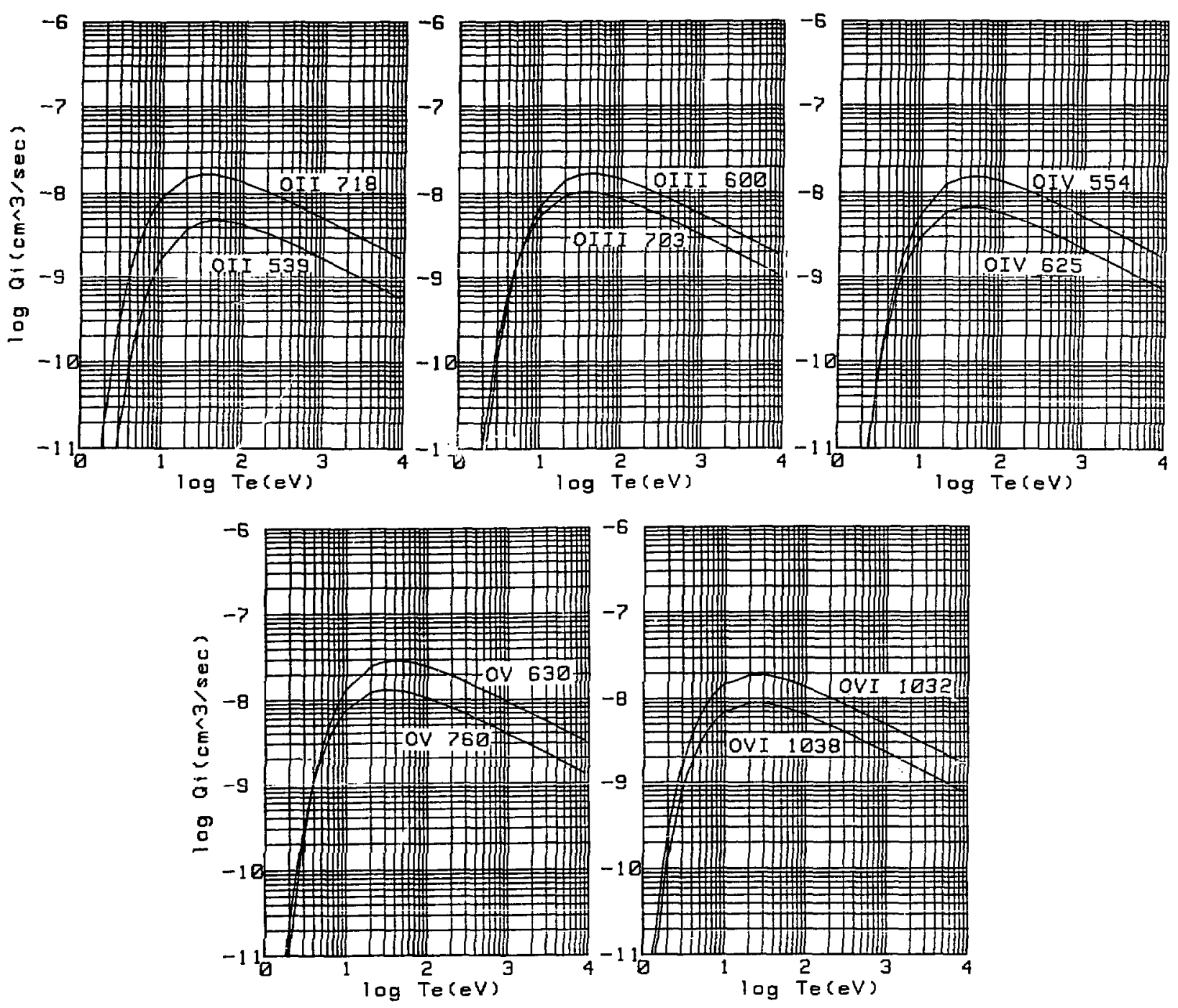

Fig. A.3. Excitation rates for transitions of oxygen ions (Gaunt factors by Davis [42]). 

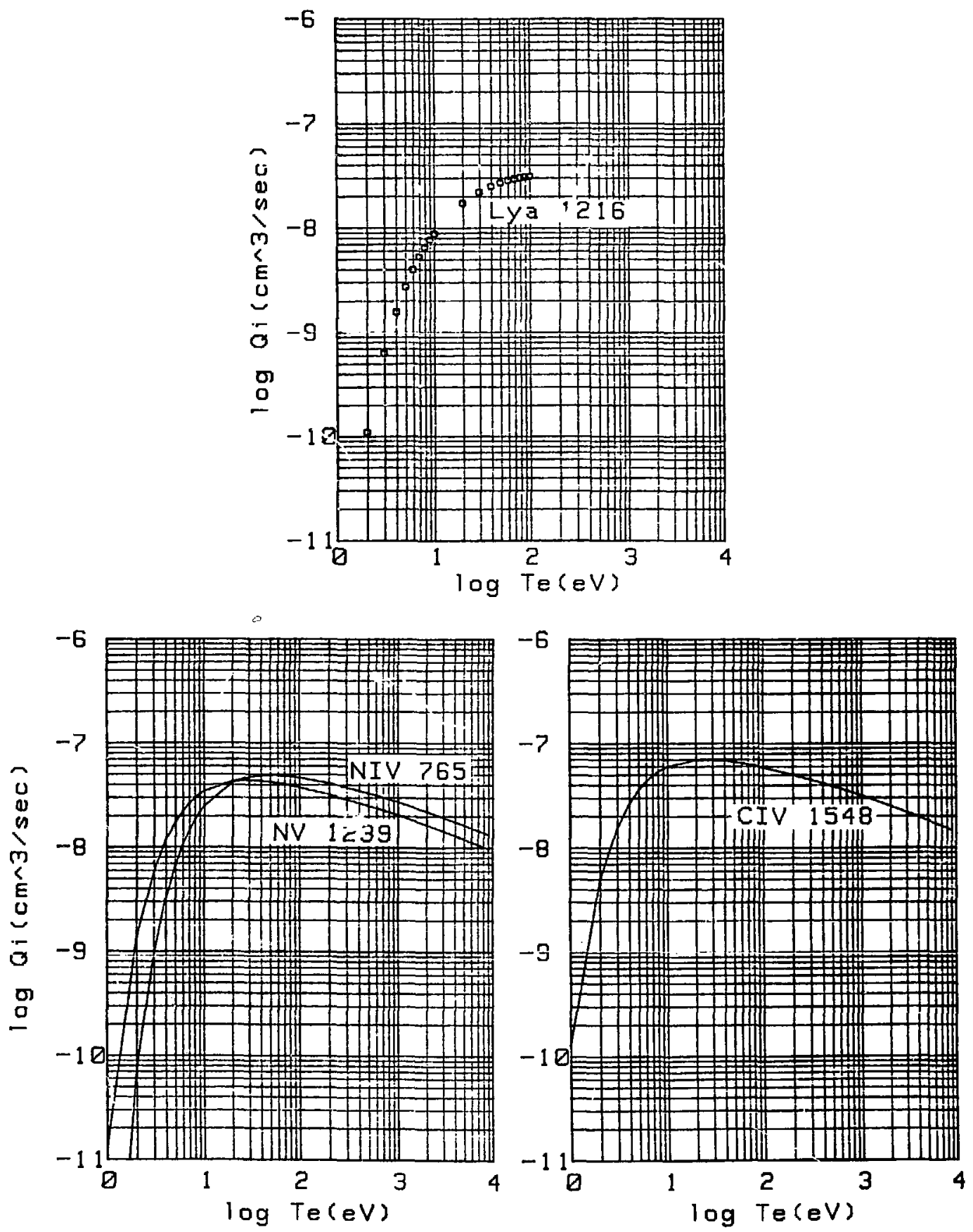

Fig. A.4. Excitation rates for Lyman aipha, N IV 765 A (LASL), NV 1239 a (LASL), C IV 1548 a (LASL). 

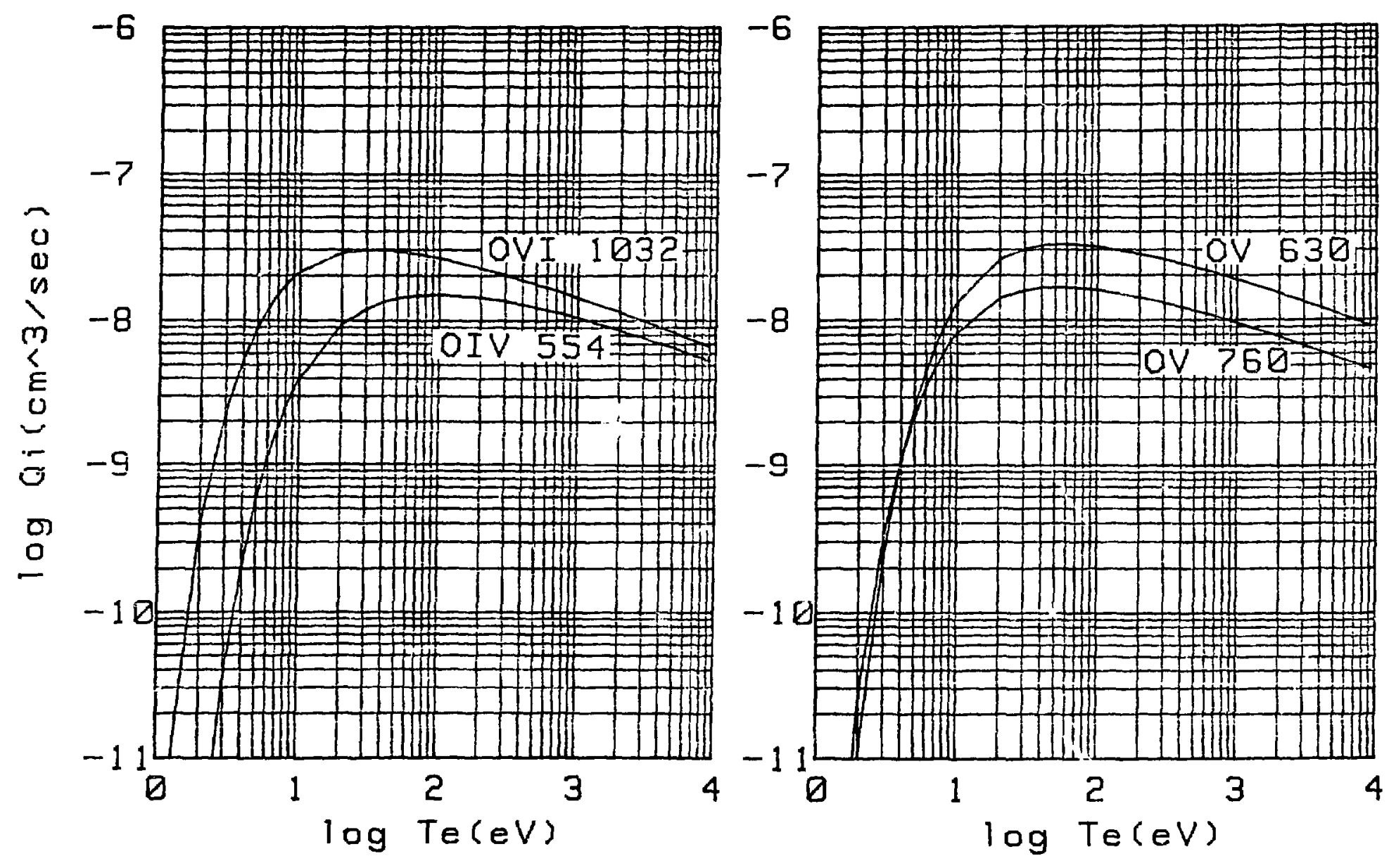

Fig. A.5. Excitation rates for transitions of oxygen ions (LASL report [39]). 

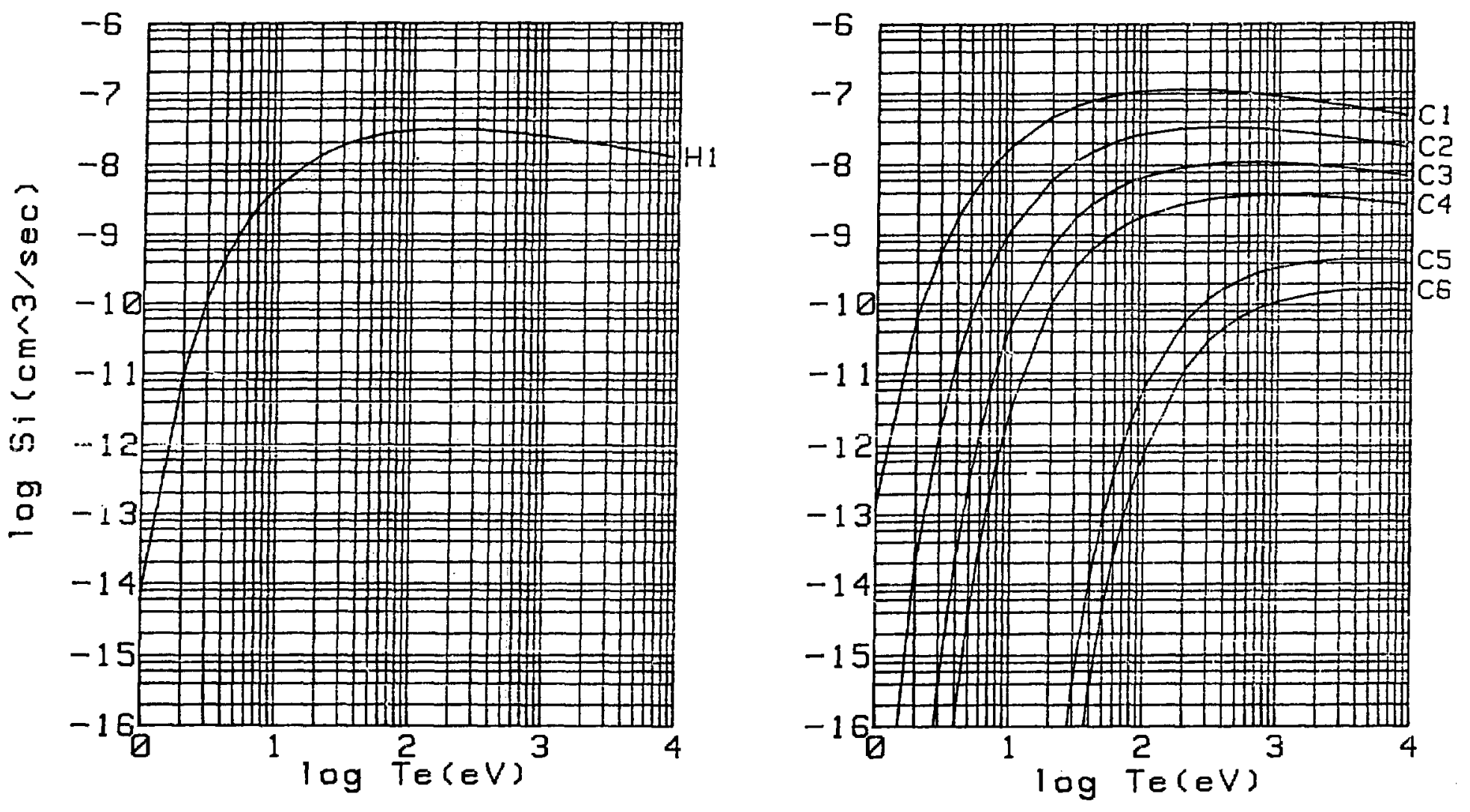

Fig. A.6. Ionization rates for deuterium and carbon (Lotz [44]). 

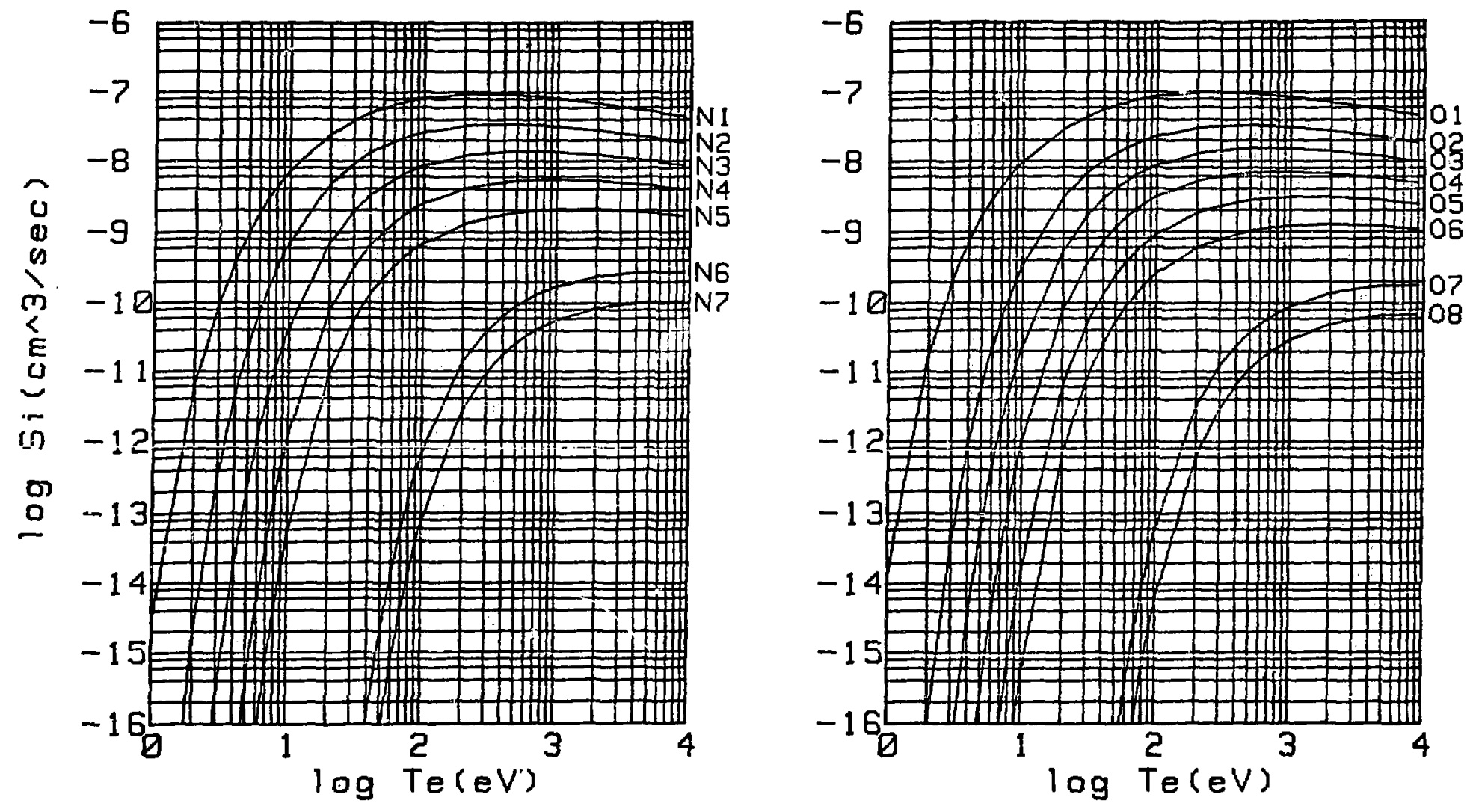

Fig. A.7. Ionization rates for nitrogen and oxygen (Lotz [44]). 


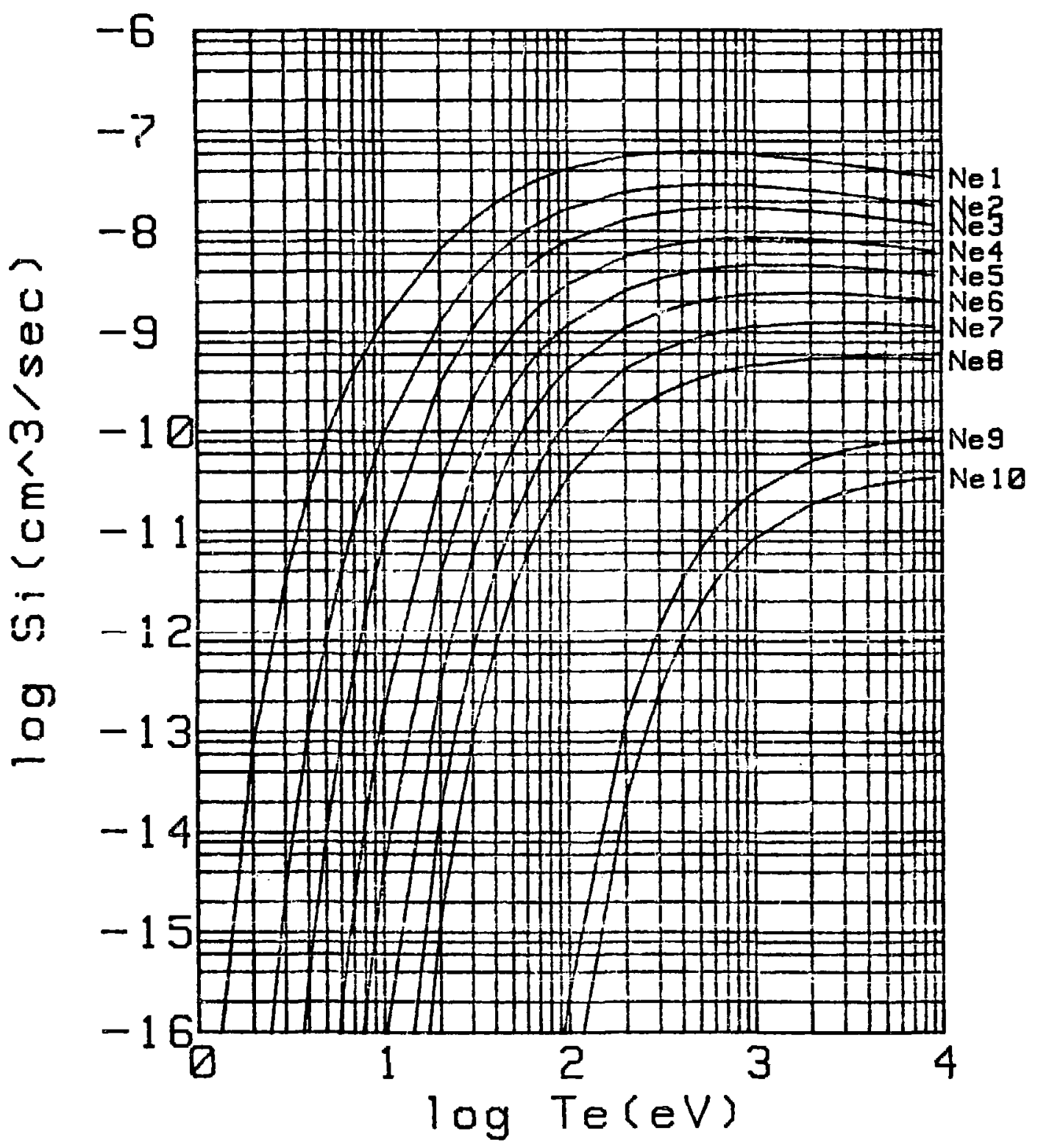

Fig. A.8. Ionization rates for neon (Lotz [44]). 

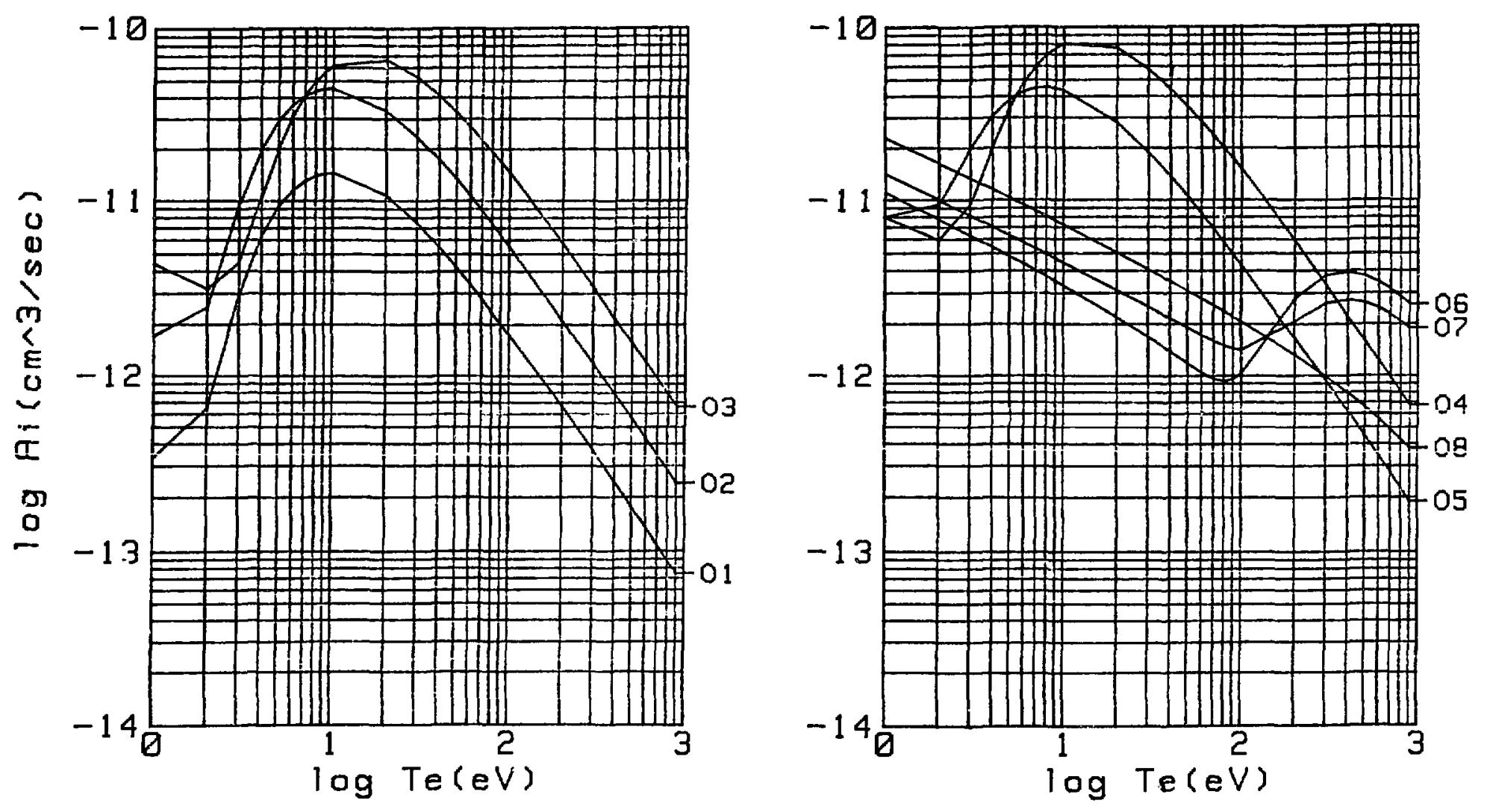

Fig. A.9. Radiative and dielectronic recombination rates for oxygen (Mattioli [46]). 
APPENDIX B: FLOWCHART OF DATA ACQUISITION, DISPLAY, AND STORAGE

As spectroscopic diagnostics become more sophisticated and gather more data per plasma shot than ever before, increasing dependence upon computer control, data acquisition, and data manipulation becomes unavoidable. Therefore, software development demands a larger fraction of a researcher's time and effort. For much of this study, two SIDS detectors, each sending 25 words of information every $500 \mu \mathrm{s}$ during a $40 \mathrm{~ms}$ TMX shot, were coupled to an HP-9845A minicomputer. During the course of this research, over 1400 TMX shots were observed with these instruments after which this data was stored and processed. This adds up to almost 5 million words (integers) of data that were recorded. A flowchart of the routine used to take data during the TMX runs is presented here. This software acquires data from two SIDS simultaneously, plots the brightnesses vs time and/or radius onto the internal printer of the HP-9845, and records the raw data onto cassette tapes. Additional information is added to the data array--such as date, species, and wavelength observed by each instrument, and slit settings used on each instrument--before the data is stored on tape. 


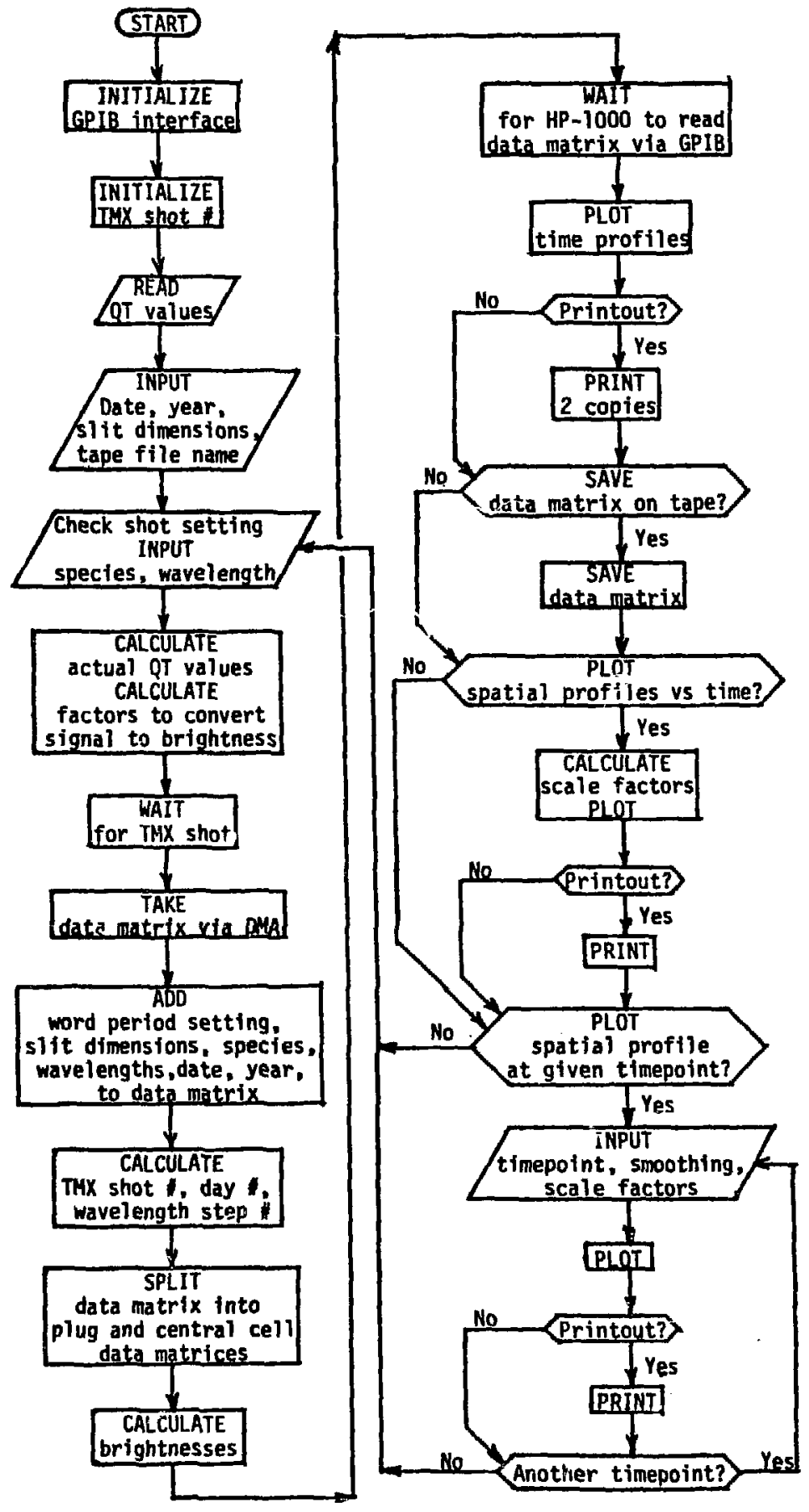


APPENGIX C: DETAILS OF THE SPATIAL-IMAGING MONOCHROMATORS

This appendix supplements Chaptar IV with a detailed description of the two spatial-imaging monochromators used on TMX. Sections 1 and 2 present the LLNL-SIDS and JHU-SIDS, respectively. The final section describes the absolute calibration procedure performed at Johns Hopkins University.

\section{C.1. THE LIVERMORE SIDS (LLNL-SIDS)}

This instrument was furchased by the Lawrence Livermore National Lasoratory for use on the TMX central cell and so it was designed to be compatible with the signal levels expected there in the wavelength range from $300 \AA$ to $1600 \AA$. The details of this instrument have not previously been written down in an organized manner, so it is done here. This section, then, is divided into several subsections. The first section discusses the details of the 0.4 monochromator design, and the second discusses details of the SIDS detector and associated electronics. The final section presents details of the calibration results.

C.1.1. The $0.4 \mathrm{~m}$ Normal Incidence Monochromator

The vacuum vessel is made of stainless steel and all seals are made of copper, including the seat of the vacuum valve which interfaces the instrument to the photon source vacuum. The instrument has its own $30 \mathrm{l} / \mathrm{s}$ ionization pump which maintains a base pressure of less than $10^{-8}$ Torr. There are no components inside the vacuum chamber which might be a source of hydrocarbons. All moving parts are either dry or lubricated with molybdenum disulfide. Furthermore, the entrance and exit slits are designed such that gas flow may occur only through the slit aperture itself. During use, typical slit dimensions are $100 \mu$ by 1 to $6 \mathrm{~mm}$, therefore, the gas conductance is very low into or out of the instrument. This greatly reduces the possibility of contamination to the monochromator by the photon source device or vice versa.

Mechanically, all $0.4 \mathrm{~m}$ instruments are designed to operate in any orientation. Available viewing port locations on fusion plasma experiments do not always allow for horizontal mounting of the instrument. Indeed, during this study, all three instruments were mounted vertically on top of TMX. 
The grating mounted in the LLNL-SIDS measures $40 \mathrm{~mm}$ by $20 \mathrm{~mm}$ with the long dimension being in the spatial imaging direction. The maximum angular field of view allowed by the grating is $f / 10$; for use on the central cell, the detector-exit slit distance set the optics at $f / 11$. The grating is coated with gold to enhance its reflectivity at short wavelengths. It has 24001 ines $/ \mathrm{mm}$, which results in a liner dispersion of $10 \AA / \mathrm{mm}$ at the slits. It was ruled by Hyperfine (\# 18-1-1) in a tripartite configuration. This means that there are three sections on the face of the grating and each is ruled with a slightly different blaze angle so that maximum reflectivity is achieved across the entire grating in its mounted geometry. The effect of the three panels can be seen in Fig. C.1, which is the instrument response vs detector anode, i.e., vs position across the grating. The blaze wavelength of the grating was designed to be at $600 \AA$ in first order in a Littrow mount.

The grating drive has several special features and has been descibed in detail elsewhere [18]. It is designed so that as it rotates the grating to change wavelengths, it also translates the grating in order to keep it on the Rowland circle, i.e., to maintain focus. Furthermore, the drive is constructed as a "sine drive." Recall the grating equation in a Littrow mount (incoming and outgoing rays have the same angle with respect to the grating normal) is $\lambda=2 d \sin \theta$, where $\lambda$ is the observed wavelength in first order, $d$ is the groove spacing, and $\theta$ is the angle between the incident light and the normal to the grating face. The linear motion of the drive screw is nearly proportional to $\sin \theta$; therefore, there is a nearly linear relationship between the observed wavelength and the motion of the drive. This simplifies the wavelength calibration, but more importantly, allows a much more accurate estimate when predicting the location of a previously unscanned emission line.

The grating drive was tested exhaustively after the LLNL-SIDS was first assembled to check the reproducibility of resetting the grating to a desired position. The drive was capable of maintaining less than a $0.07 \AA$ uncertainty in the wavelength setting. This is sufficient since the slits were typically set for 1 \& resolution during these experiments.

\section{C.1.2. The Spatial Imaging Detector System (SIDS)}

The heart of the spatial imaging detector is composed of two $2.54 \mathrm{~cm}$ diameter microchannel plates (MCP's) made by Galileo. They are parallel to each other such that the microchannels form a chevron, or vee, design (see 


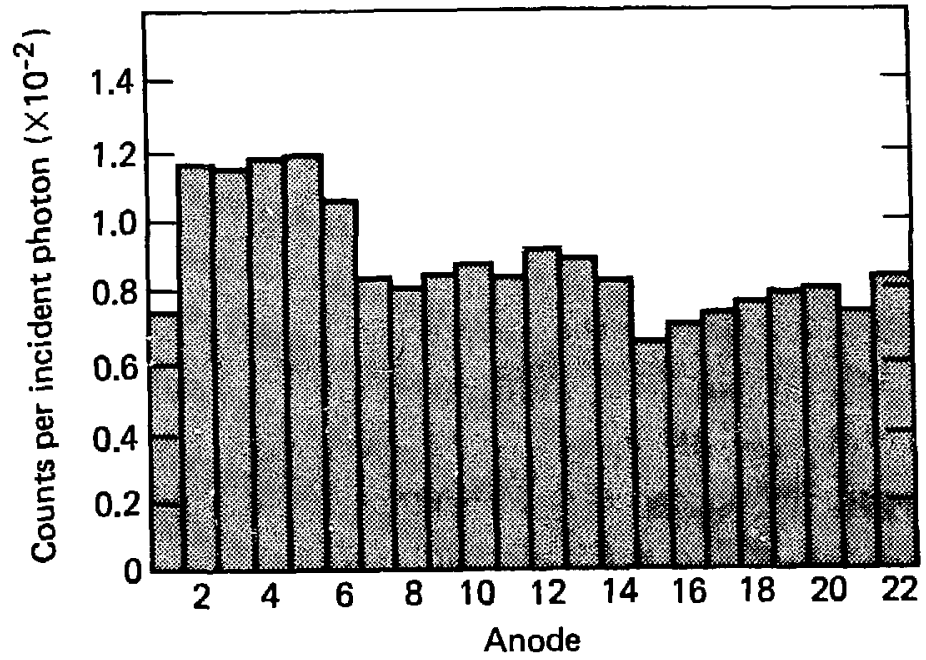

Fig. C.1. Quantum efficiency of the SIDS detector times the transmission of the grating (QT) vS anode in the LLNL-SIDS instrument at $735 \mathrm{~A}$. The effect of the three panels of the grating can be seen. 
Fig. (.2). This helps to assure that the photons will strike the microchannel near the front surface in order to achieve maximum gain from each photoevent and thus provides a better quality pulse height distribution emerging from the second plate. MCP's are known to start saturating when the signal current approaches $10 \%$ of the strip current. (The strip current is the resistive current which flows through the plates due to the applied high voltage.) Each MCP was specially made to have a high strip current-- $0.87 \mathrm{\mu A} / \mathrm{cm}^{2}$ at $1000 \mathrm{~V}$--so that these MCP's can tolerate a high signal level without saturating. These plates were operated at about $600 \mathrm{~V}$ each to provide a total gain of $4 \times 10^{5}$.

The front surface of the first plate is coated with approximately $1000 \AA$ of CuI. This helps to enhance the quantum efficiency at wavelengths above $1200 \AA$. Furthermore, a hollow cylinder biased at about $-100 \mathrm{~V}$ is mounted in front of the first plate so that no electrons are lost in the forward direction.

Approximately $100 \mu$ behind the second MCP are the spatial imaging anodes. They are biased slightly positive with respect to the MCP and rely on proximity focusing to collect the electrons. The 22 anodes are made of aluminum strips which are $525 \mu$ wide and spaced $635 \mu$ apart center-to-center. They fill the entire active area behind the MCP, so that the outer anodes are shorter than the central anodes. Each anode is connected through a ceramic vacuum seal to its own pulse amplifier discriminator (PAD) located outside the vacuum.

The PAD's are mounted on an aluminum plate which serves as the ground plane for all of them. These PAD's were specially designed and built by E. Mackey of Spacom Electronics, Camarillo, CA, to have a very low threshold of $2 \times 10^{-14}$ coul/pulse; this corresponds to $1.2 \times 10^{5}$ electrons/pulse. Therefor: : with a total MCP gain of $4 \times 10^{5}$, nearly every photoevent results in a 3 volt, $250 \mu \mathrm{s}$ TTL pulse from the PAD output. These TTL pulses are sent through 22 coaxial cables to the data conversion unit in the diagnostic room where they are counted for a specified time period, added to information words and then sent serially via direct memory access to an HP-9845 minicomputer. The computer processes the data, displays it on a CRT and stores it on magnetic tape.

\section{C.1.3. Calibration Results of the LLNL-SIDS}

This section contains a brief description of many details of the LLNL-SIDS . operation and calibration. It is mainly intended to provide a source of 


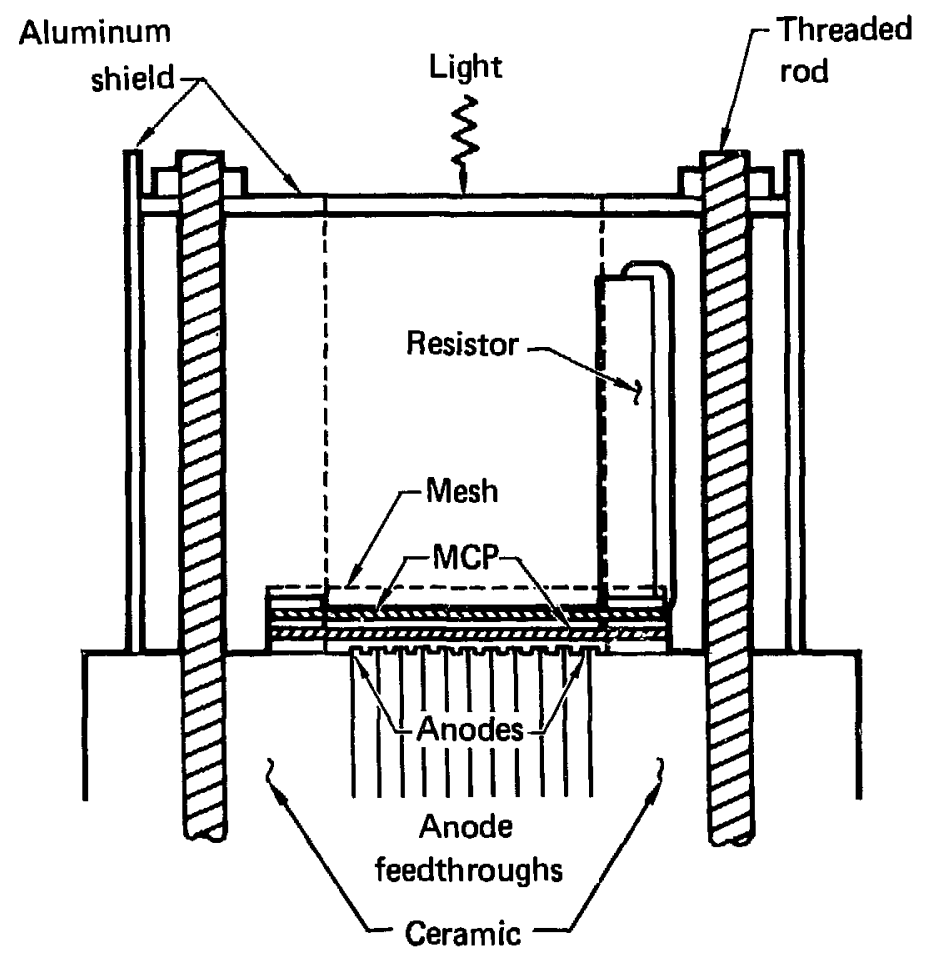

Fig. C.2. Schematic diagram of the Spatial Imaging Detector System (SIDS). 
information for this instrument, since its description will not be published elsewhere.

1. Photometric calibration. Figure C.3 plots quantum efficiency times grating transmission (QT), that is, the total LLNL-SIDS response for anode 11 both before and after the experiment. Table C.l lists all QT values obtained after the experiment on TMX. The values for $300 \AA$ were obtained by dividing the numbers at $406 \AA$ by 10.

2. Reflectivity of the grating. Figure C.4 shows the reflectivity of the center panel of the grating in the LLNL-SIDS. The grating is blazed at $600 \AA$ in first order so that the first order transmission is peaked at that wavelength. Furthermore, the transmission remains quite high at increasing wavelength, while all other orders are suppressed. 0nly zero order increases significantly at higher wavelengths. The behaviors of the other two panels are similar.

3. Scattered light rejection (Fig. C.5). During this test, monochromatic light at $584 \AA$ was allowed to pass into the LLNL-SIDS. (Recall that the grating is blazed at $600 \AA$.) Then the grating drive was scanned and the count rate was measured at various points throughout the wavelength range of the instrument. The values on the vertical axis of the graph are normalized to the count rate at the peak of $584 \mathrm{~A}$.

4. Detector counts vs incident photon flux. The detector counting rate $R$ is given by

$$
R=\frac{J A}{e-G}
$$

where $J$ is the current density due to the signal only, $A$ is the area of a given ancae, $e$ is the electron charge and $G$ is the total gain of the two MCP's. The saturation 1 imit can be estimated by letting $J$ equal $10 \%$ of the strip current density. Therefore, $\mathrm{R}_{\max } \sim 2 \times 10^{5}$ counts/s per anode before saturation effects become important. The graph in Fig. C.6 corresponds to $10^{5}$ counts/s on anode 14 and is still linear as expected.

5. Wavelength calibration. Since the wavelength drive is constructed as a sine drive, there is a nearly linear relationship between the step number reading and the wavelength. Plotting the step number to wavelength ratio vs wavelength should yield a nearly constant value. With a $2400 \mathrm{line} / \mathrm{mm}$ grating, the wavelength drive needs to advance approximately 30 steps to scan a $1 \AA$ spectral width past the exit slit. Figure C.7 gives the wavelength calibration used on TMX. 


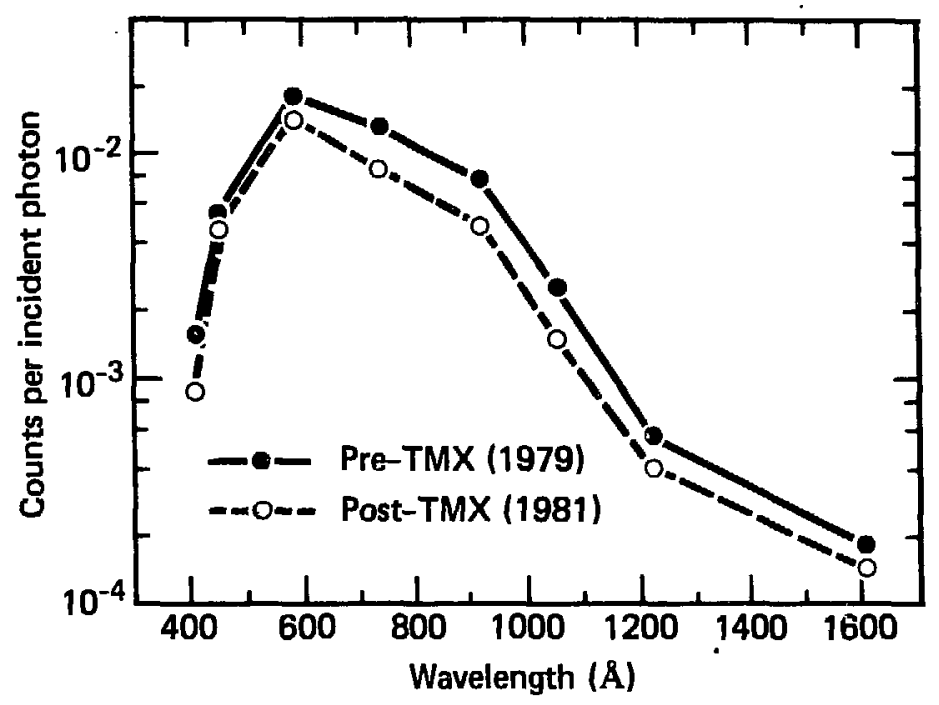

Fig. C.3. Photometric calibration vs wavelength for anode 11 of the LLNL-SIDS before and after the TMX experiment. 
Table C.1. QT values vs anode for LLNL-SIDS.

\begin{tabular}{lcccccccc} 
LAMBDA & 1 & 2 & 3 & 4 & 5 & 6 & 7 & 8 \\
\hline 390 & $3.53(-5)$ & $8.04(-5)$ & $5.53(-5)$ & $4.14(-5)$ & $4.28(-5)$ & $7.92(-5)$ & $1.12(-4)$ & $1.87(-4)$ \\
496 & $3.53(-4)$ & $8.04(-4)$ & $5.53(-4)$ & $4.14(-4)$ & $4.28(-4)$ & $7.92(-4)$ & $1.12(-3)$ & $1.07(-3)$ \\
468 & $2.72(-3)$ & $5.62(-3)$ & $4.72(-3)$ & $3.75(-3)$ & $3.53(-3)$ & $5.08(-3)$ & $6.31(-3)$ & $6.81(-3)$ \\
584 & $9.81(-3)$ & $1.75(-2)$ & $1.75(-2)$ & $1.68(-2)$ & $1.59(-2)$ & $1.47(-2)$ & $1.41(-2)$ & $1.37(-2)$ \\
735 & $7.43(-3)$ & $1.16(-2)$ & $1.15(-2)$ & $1.18(-2)$ & $1.19(-2)$ & $1.06(-2)$ & $8.27(-3)$ & $8.84(-3)$ \\
928 & $1.44(-3)$ & $7.28(-3)$ & $7.64(-3)$ & $7.95(-3)$ & $8.22(-3)$ & $6.74(-3)$ & $4.55(-3)$ & $4.37(-3)$ \\
1048 & $1.63(-3)$ & $6.67(-3)$ & $4.58(-3)$ & $2.77(-3)$ & $2.91(-3)$ & $2.33(-3)$ & $1.54(-3)$ & $1.45(-3)$ \\
1216 & $4.36(-4)$ & $8.41(-4)$ & $7.59(-4)$ & $7.29(-4)$ & $7.85(-4)$ & $7.00(-4)$ & $4.32(-4)$ & $4.10(-4)$ \\
1698 & $4.21(-5)$ & $1.81(-4)$ & $2.18(-4)$ & $2.28(-4)$ & $2.44(-4)$ & $2.26(-4)$ & $1.57(-4)$ & $1.32(-4)$
\end{tabular}

\begin{tabular}{|c|c|c|c|c|c|c|c|c|}
\hline AMB & 9 & 18 & 11 & 12 & 13 & 14 & 15 & 16 \\
\hline $\begin{array}{l}388 \\
486 \\
468 \\
584 \\
735 \\
928 \\
1848 \\
1216 \\
2688\end{array}$ & $\begin{array}{l}9.50(-5) \\
9.50(-4) \\
5.49(-3) \\
1.39(-2) \\
8.37(-3) \\
4.67(-3) \\
1.56(-3) \\
4.18(-4) \\
1.23(-4)\end{array}$ & $\begin{array}{l}1.26(-4) \\
1.26(-3) \\
6.24(-3) \\
1.53(-2) \\
8.66(-3) \\
4.65(-3) \\
1.52(-3) \\
4.52(-4) \\
1.19(-4)\end{array}$ & $\begin{array}{l}9.58(-5) \\
9.58(-4) \\
5.49(-3) \\
1.39(-2) \\
8.37(-3) \\
4.67(-3) \\
1.56(-3) \\
4.29(-4) \\
1.28(-4)\end{array}$ & $\begin{array}{l}8.25 \\
8.25 \\
6.45 \\
1.42 \\
9.85 \\
5.45 \\
2.9 \\
5.38 \\
1.5\end{array}$ & $\begin{array}{l}5.9 \\
5.9 \\
3.8 \\
1.3 \\
8.8 \\
5.3 \\
1.7 \\
4.7 \\
1.5\end{array}$ & $\begin{array}{l}0.4 \\
8.4 \\
4.8 \\
1.2 \\
8.1 \\
4.9 \\
1.6 \\
4.2 \\
1.5\end{array}$ & $\begin{array}{l}1.15(-4) \\
1.15(-3) \\
5.01(-3) \\
1.15(-2) \\
6.46(-3) \\
3.51(-3) \\
1.14(-3) \\
3.84(-4) \\
1.26(-4)\end{array}$ & $\begin{array}{l}1.12(-4) \\
1.12(-3) \\
5.86(-3) \\
1.38(-2) \\
6.98(-3) \\
3.98(-3) \\
1.19(-3) \\
3.38(-4) \\
1.17(-4)\end{array}$ \\
\hline
\end{tabular}

\begin{tabular}{lcccccc} 
LAMBDA & 17 & 18 & 19 & 20 & 21 & 22 \\
\hline 380 & $1.85(-4)$ & $8.36(-5)$ & $7.58(-5)$ & $7.19(-5)$ & $7.38(-5)$ & $5.82(-5)$ \\
486 & $1.85(-3)$ & $9.36(-4)$ & $7.58(-4)$ & $7.19(-4)$ & $7.38(-4)$ & $6.82(-4)$ \\
460 & $5.85(-3)$ & $8.54(-3)$ & $4.26(-3)$ & $3.77(-3)$ & $3.91(-3)$ & $3.92(-3)$ \\
584 & $1.24(-2)$ & $1.23(-2)$ & $1.18(-2)$ & $1.14(-2)$ & $1.18(-2)$ & $1.13(-2)$ \\
735 & $7.26(-3)$ & $7.57(-3)$ & $7.87(-3)$ & $7.95(-3)$ & $7.37(-3)$ & $8.31(-3)$ \\
920 & $4.62(-3)$ & $4.38(-3)$ & $4.74(-3)$ & $4.61(-3)$ & $4.24(-3)$ & $4.48(-3)$ \\
1848 & $1.23(-3)$ & $1.34(-3)$ & $1.54(-3)$ & $1.45(-3)$ & $1.38(-3)$ & $1.44(-3)$ \\
1216 & $3.48(-4)$ & $3.66(-4)$ & $3.82(-4)$ & $4.08(-4)$ & $3.61(-4)$ & $3.53(-4)$ \\
1688 & $1.17(-4)$ & $1.38(-4)$ & $1.43(-4)$ & $1.33(-4)$ & $1.22(-4)$ & $1.33(-4)$
\end{tabular}




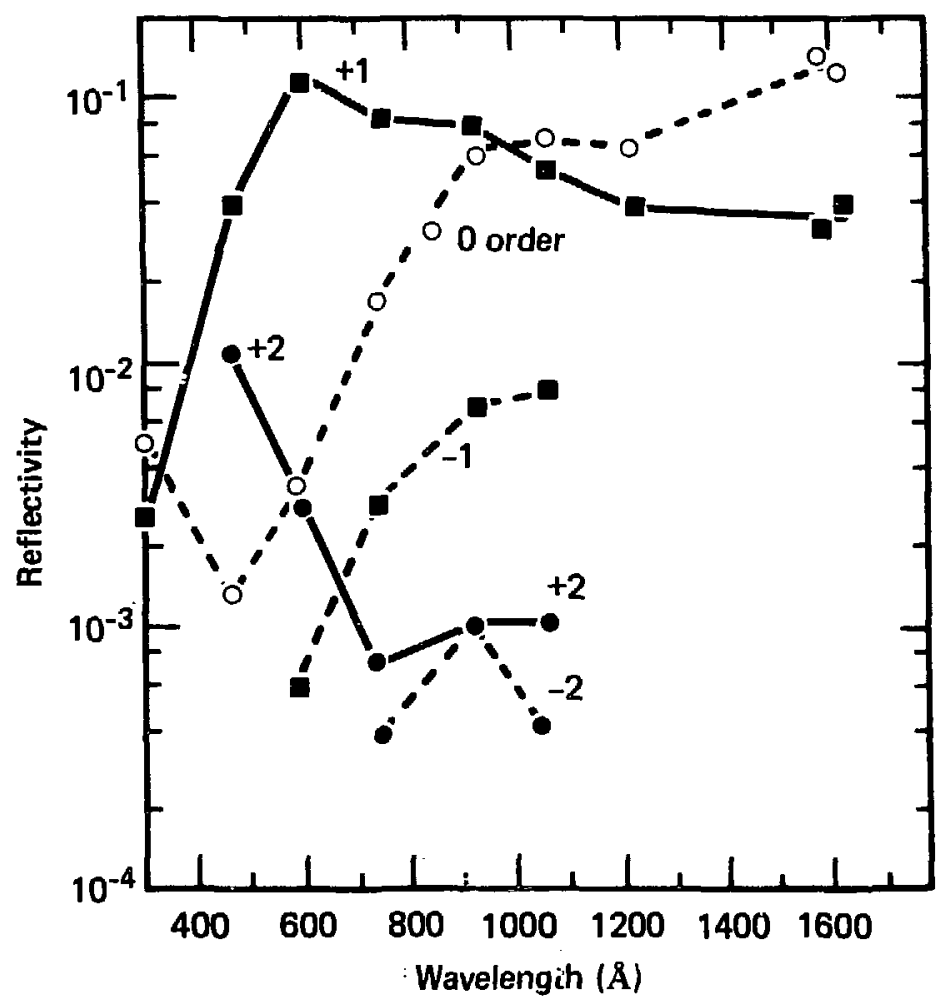

Fig. C.4. Reflectivity of the center panel of the grating in the LLNL-SIDS vs wavelength and order. This grating is blazed at $600 \AA$ in first order. 


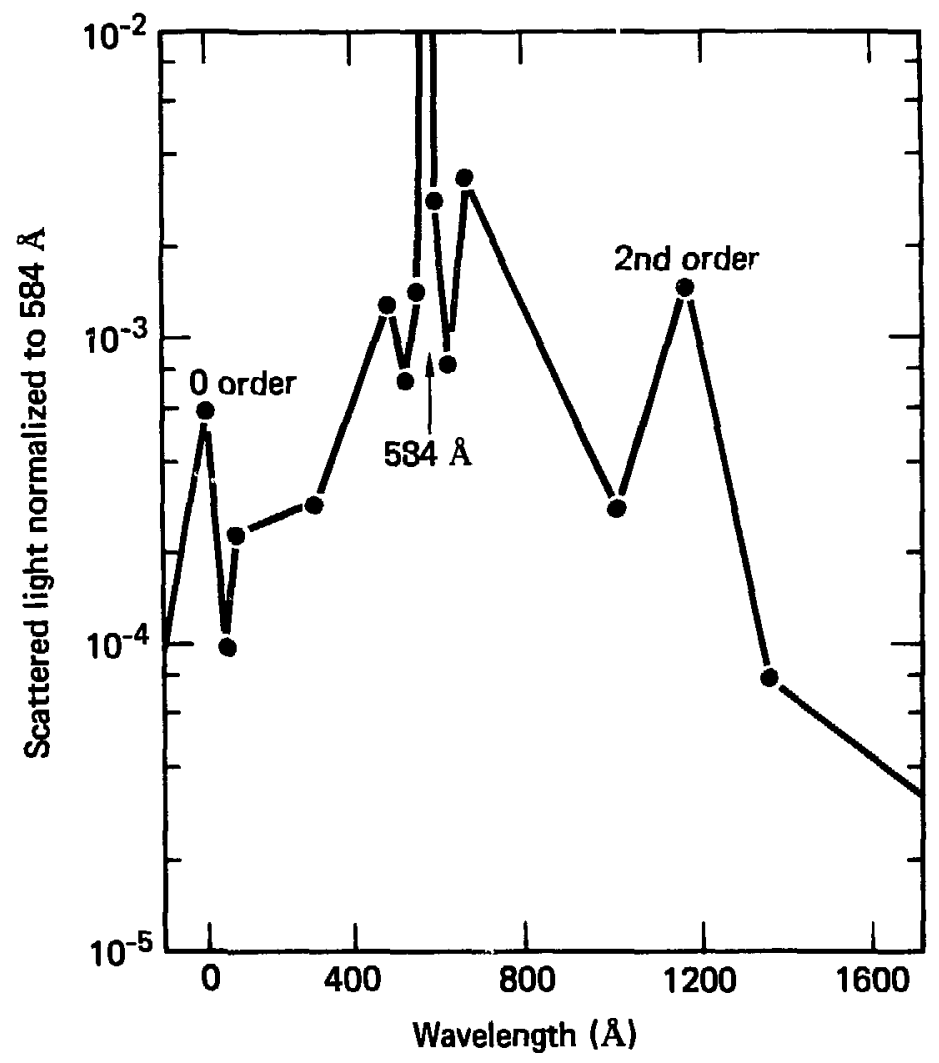

Fig. C.5. Rejection of scattered light by the LLNL-SIDS at $584 \mathrm{~A}$. The values are normalized to the signal obtained at 584 A. Note the increase at second order. 


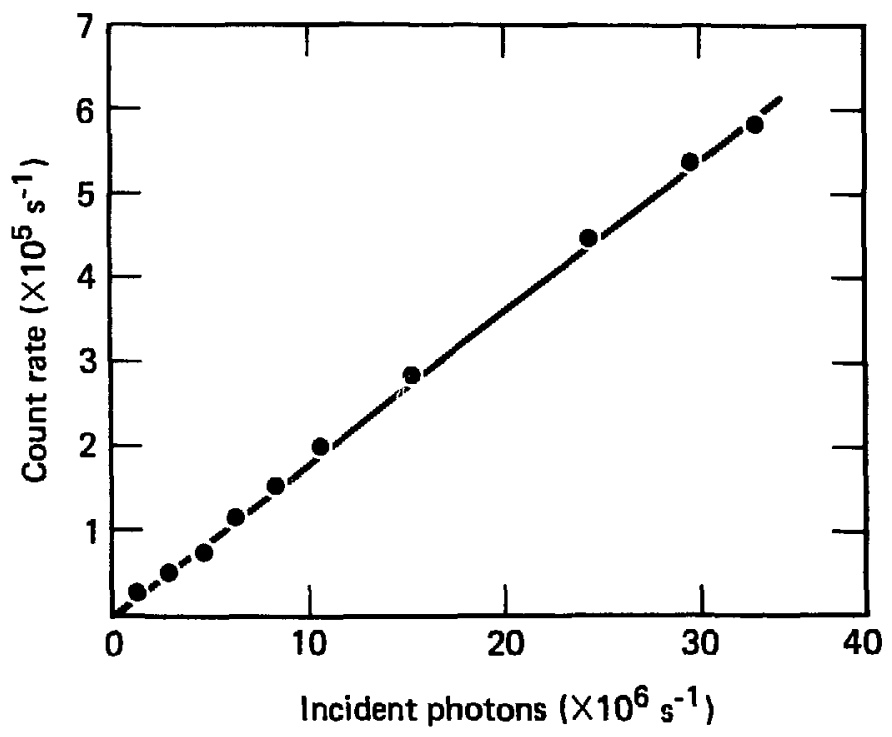

Fig. C.6. LLNL-SIDS detector counting rate vs incident photon flux at $584 \AA$. The response of the detector is still linear at $10^{5}$ counts per second per anode. 


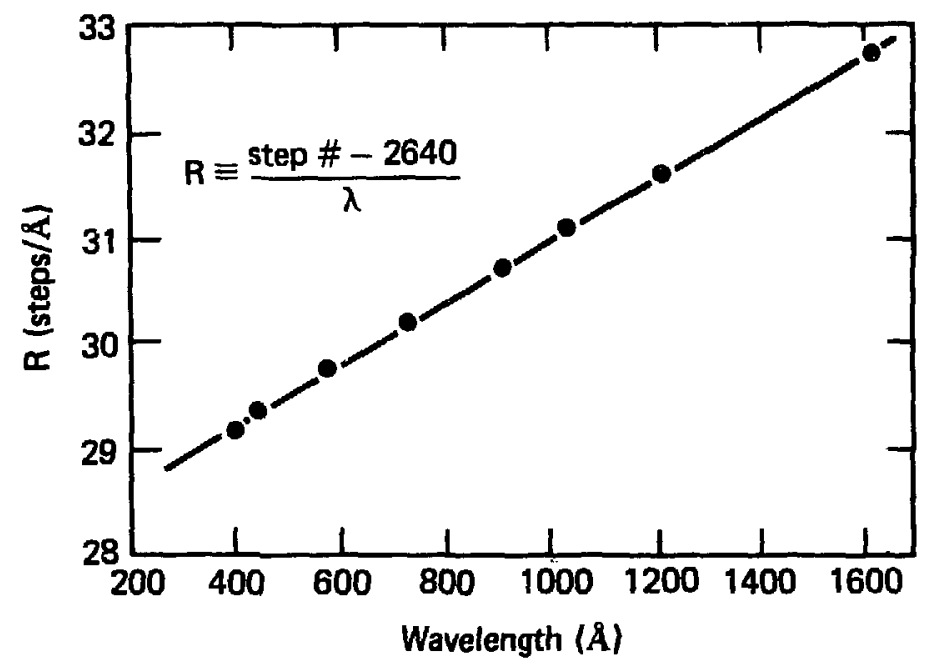

Fig. C.7. Wavelength calibration of the LLNL-SIDS instrument. The dispersion of the grating results in approxiriately 30 steps of the wavelength drive per $\AA$. 
6. Entrance and exit slit calibrations. Figure $\mathcal{C} .8$ plots the entrance slit width and height and the exit slit width vs micrometer setting. Both slits have only one movable jaw; the other is fixed. The exit slit height is fixed at $6 \mathrm{~mm}$. The entrance slit height is adjusted by translating a wedge past the opening, therefore, the entrance slit is actually trapezoidal in shape rather than rectangular. The height given on the graph is an effective height which, when multiplied times the width, will yield the correct entrance slit area.

7. Analysis of MCP circuit and MCP gain. Table C.2 lists values comparing applied high voltage with strip current and voltage per plate along with the gains for two voltages (per plate) as determined by Galileo. The high voltage applied during operation was $2680 \mathrm{~V}$ which resulted in $616 \mathrm{~V}$ per MCP and a strip current of $12 \mu \mathrm{A}(4.6 \mu \mathrm{A} \mathrm{cm}-2)$.

8. Detector counts vs applied high voltage (Fig. C.9). The increased count rate with high voltage is due to the increased gain of the MCP's. If the instrument is not operated on High Voltage Setting \#5, then all QT values must be corrected according to the factor indicated by Fig. C.9.

9. Magnetic shield test. After the magnetic shield was mounted around the detector and the electronics, the system was exposed to 600 gauss d.c. magnetic fields from all possible directions. No effects on the signal were observed at any time.

\section{C.2. THE JOHNS HOPKINS UNIVERSITY SIDS (JHU-SIDS)}

The JHU-SIDS is very similar to the LLNL-SIDS and so is not discussed in detail here. It has, however, been described thoroughly elsewhere $[15,20]$. This instrument is the combination of a $0.4 \mathrm{~m}$ normal incidence monochromator with the original spatial imaging detector built at JHU. It was designed for the higher signal levels expected on tokamaks, so some of the details are slightly different from the LLNL-SIDS. These are mentioned briefly here.

The $0.4 \mathrm{~m}$ monochromator is essentially identical to the one on the LLNL-SIDS except for the grating. This gold-coated grating has the same physical dimensions $(40 \mathrm{~mm} \times 20 \mathrm{~mm})$, and is also tripartite. However, it was ruled by Hyperfine with 3600 lines/mm alı is blazed at $300 \AA$. This smaller groove spacing means that the linear dispersion is greater and so the slits must be set at larger widths to achieve a given spectral resolution, e.g., $1 \AA$ slits are $150 \mu$ wide on the JHU-SIDS vs $100 \mu$ wide on the LLNL-SIDS. 

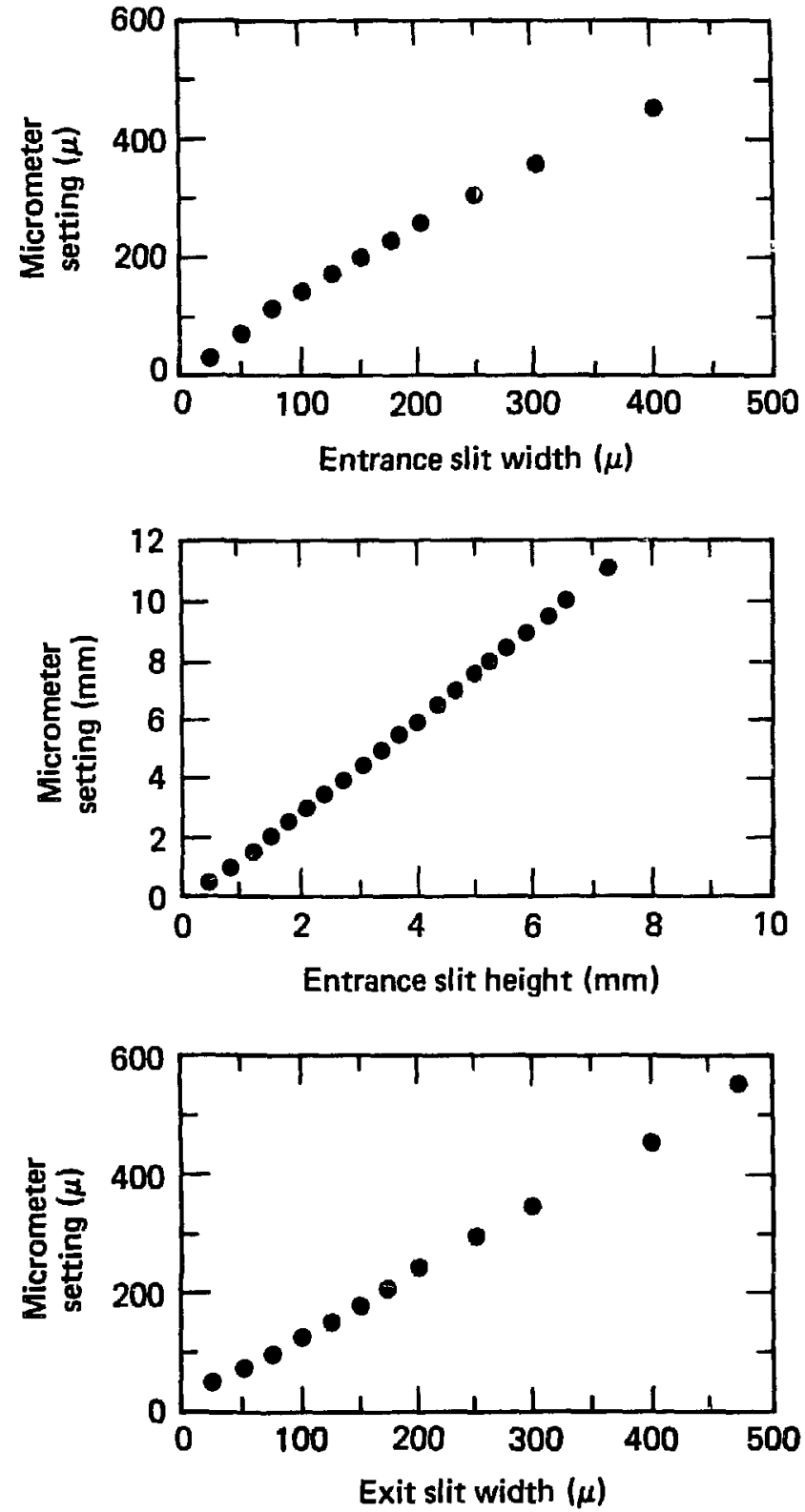

Fig. C.8. Calibration of the LLNL-SIDS entrance and exit slit widths and entrance slit height. Slit widths of $100 \mu$ corresponds to 1 A resolution. 
Table C.2. Strip current, voltage, and gain per MCP of LLNL-SIDS for various voltages from high voltage supply.

\begin{tabular}{lcccc}
\hline HV (volts) & $I_{S}(\mu A)$ & $V_{p l a t e}($ volts) & 1st MCP & 2nd MCP \\
\hline 1000 & 4.4 & 230 & & \\
2000 & 8.8 & 459 & & \\
2680 & 11.7 & 616 & & \\
3043 & 13.4 & 700 & $4.7 \times 10^{2}$ & $8.7 \times 10^{2}$ \\
4000 & 17.7 & 918 & & \\
4348 & 19.1 & 1000 & $2.3 \times 10^{4}$ & $2.5 \times 10^{4}$ \\
\hline
\end{tabular}




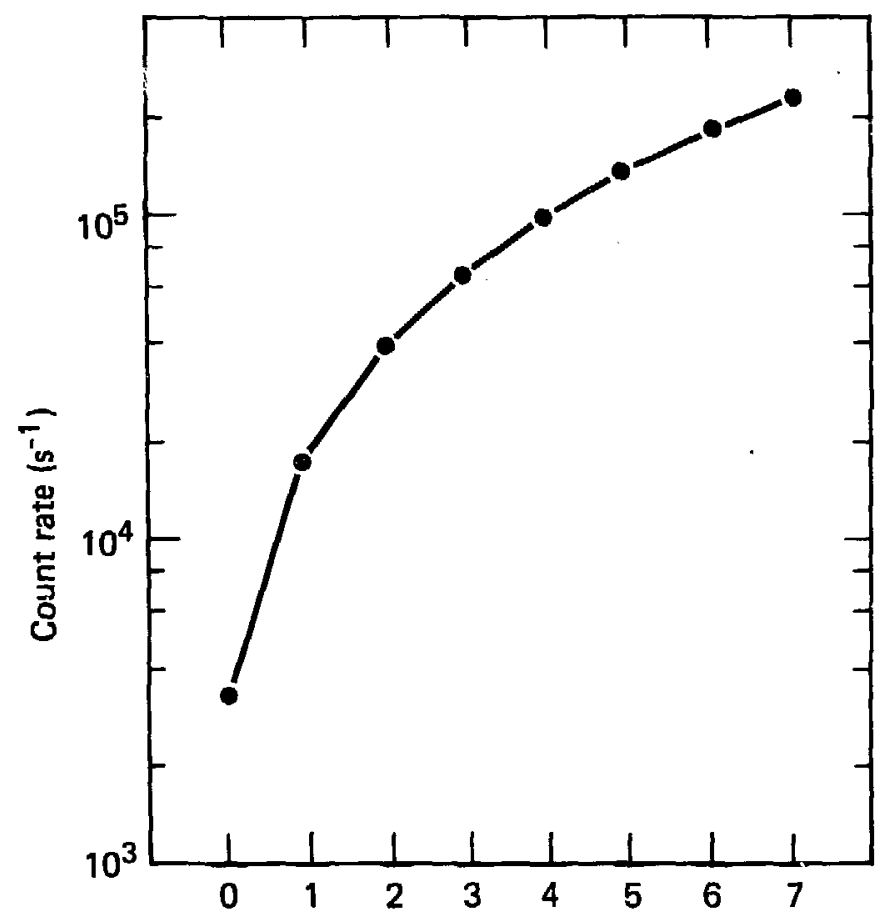

High voltage setting

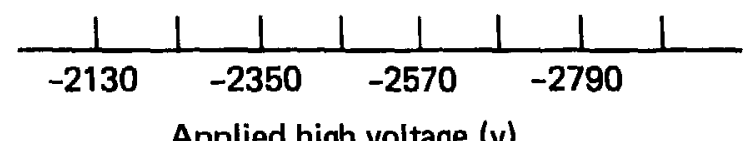

Applied high voltage (v)

Fig. C.9. LLNL-SIDS detector count rate vs applied high voltage. 
Another difference between the two instruments is in the spatial imaging optics. The distance between the exit slit and the detector is greater than on the LLNL-SIDS so that the total angular field of view is $f / 19$ rather than $\mathrm{f} / \mathrm{ll}$. The smaller field of view is more compatible with the smaller plasma radius found on Alcator A where the JHU-SIDS was first used, and so was well suited for the plasma radius in the TMX end cells.

The physical dimensions of the SIDS detector itself are identical to those on the LLNL-SIDS. However, the microchannel plates (MCP's) were made by Varian ( 8900 series) and the first plate is not coated. The first plate is a standard low strip current plate but the second plate was built for high strip currents $\left(7 \mu \mathrm{A} / \mathrm{cm}^{2}\right)$. The total gain is approximately $10^{5}$ for both plates. The PAD threshold for each anode is set at $7 \times 10^{4}$ electrons. Every other aspect of the JHU-SIDS is identical to that of the LLNL-SIDS. In fact, each instrument could be run by the same HP-9845 computer with no change in software; eventually, both instruments were run simultaneously by the HP-9845.

The photometric calibration of the JHU-SIDS is shown in Fig. C.10. It can be seen that these values are approximately a factor of 10 lower than those of the LLNL-SIDS. This allows the JHU-SIDS to observe higher signal levels without saturation. Finally, the complete set of QT values obtained during the post-experiment calibration is given in Table C.3.

\section{C.3. THE CALIBRATION PROCEDURE}

The plasma spectroscopy group at JHU has designed and built a calibration facility [17] which was used to calibrate the equipment used in this study. Known as the extreme ultraviolet calibration equipment (ECE), it has a usable wavelength range of $300 \AA$ to $1600 \AA$. A schematic drawing of the ECE is given in Fig. C.11. The heart of this facility is a photodiode detector which has been absolutely calibrated for photometric response by the National Bureau of Standards [48]. The source of photons on the ECE is a hollow cathode lamp through which various gases are allowed to flow in order to obtain different emission lines from excited neutrals or singly ionized species. The desired wavelength is selected by the premonochromator which is actually another $0.4 \mathrm{~m}$ normal incidence monochromator. The monochromatic beam then passes to a mirror which can be tilted to direct the beam either onto the reference diode or into the instrument to be calibrated. The tilting mirror is concave so that it can focus an image of the premonochromator exit slit to a location 


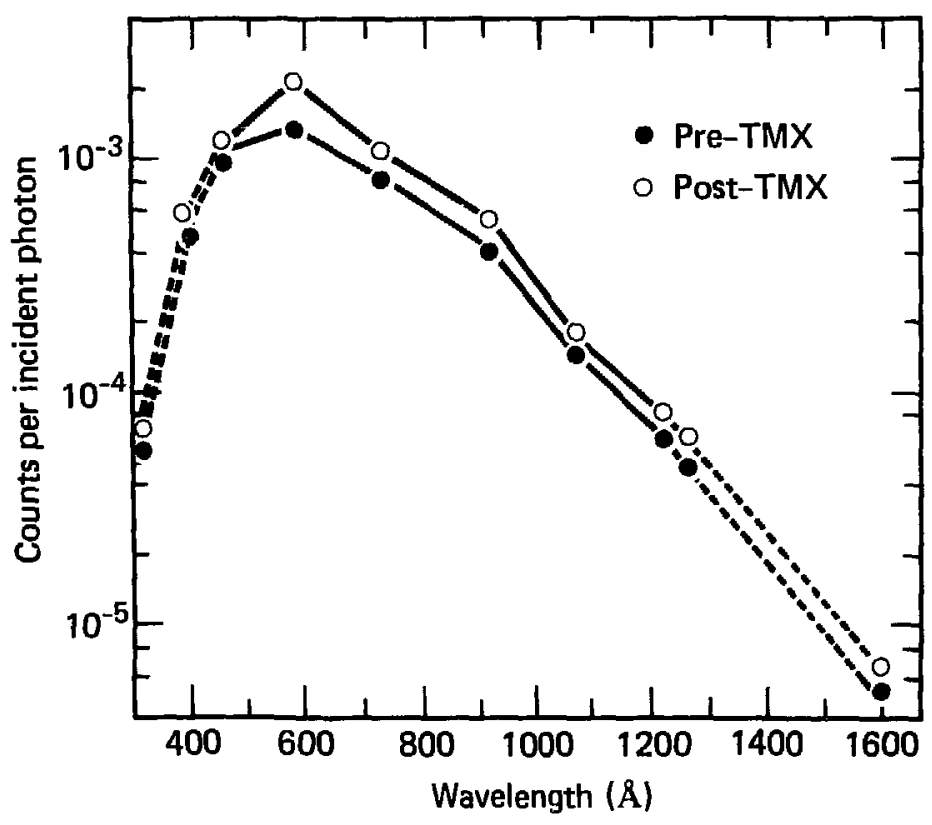

Fig. C.10. Photometric calibration vs wave length for anode 11 of the JHU-SIOS before and after the TMX experiment. 
Table C.3. QT values vs anode for JHU-SIDS.

\begin{tabular}{|c|c|c|c|c|c|c|c|c|}
\hline LAMEDA & 1 & 2 & 3 & 4 & 5 & 6 & $?$ & 8 \\
\hline $\begin{array}{l}300 \\
400 \\
468 \\
584 \\
735 \\
932 \\
1866 \\
1216 \\
1255 \\
1680\end{array}$ & $\begin{array}{l}4.40(-5) \\
4.40(-4) \\
8.80(-4) \\
1.69(-3) \\
7.75(-4) \\
3.93(-4) \\
1.33(-4) \\
4.53(-5) \\
3.62(-5) \\
3.62(-6)\end{array}$ & $\begin{array}{l}3.68(-5) \\
3.68(-4) \\
7.36(-4) \\
1.39(-3) \\
6.48(-4) \\
3.46(-4) \\
1.15(-4) \\
3.33(-5) \\
2.66(-5) \\
2.66(-6)\end{array}$ & $\begin{array}{l}4.67(-5) \\
4.67(-4) \\
9.35(-4) \\
1.59(-3) \\
7.94(-4) \\
4.00(-4) \\
1.30(-4) \\
3.70(-5) \\
2.96(-5) \\
2.96(-6)\end{array}$ & $\begin{array}{l}3.63(-5) \\
3.63(-4) \\
7.27(-4) \\
1.35(-3) \\
6.49(-4) \\
2.97(-4) \\
1.01(-4) \\
4.26(-5) \\
3.48(-5) \\
3.48(-6)\end{array}$ & $\begin{array}{l}3.29(-5) \\
3.29(-4) \\
6.58(-4) \\
1.18(-3) \\
5.39(-4) \\
2.79(-4) \\
9.33(-5) \\
3.05(-5) \\
2.44(-5) \\
2.44(-6)\end{array}$ & $\begin{array}{l}6.97(-5) \\
6.97(-4) \\
1.39(-3) \\
2.15(-3) \\
4.85(-4) \\
2.26(-4) \\
1.18(-4) \\
3.51(-5) \\
2.8 B(-5) \\
2.8 B(-6)\end{array}$ & $\begin{array}{l}0.19(-5) \\
0.19(-4) \\
1.63(-3) \\
2.70(-3) \\
1.60(-3) \\
6.76(-4) \\
1.70(-4) \\
6.27(-5) \\
5.02(-5) \\
5.82(-6)\end{array}$ & $\begin{array}{l}6.42(-5) \\
6.42(-4) \\
1.28(-3) \\
2.65(-3) \\
1.29(-3) \\
7.13(-4) \\
2.38(-4) \\
9.61(-5) \\
7.69(-5) \\
7.69(-6)\end{array}$ \\
\hline
\end{tabular}

\begin{tabular}{|c|c|c|c|c|c|c|c|c|}
\hline LAMBDA & 9 & 10 & 11 & 12 & 13 & 14 & 15 & 16 \\
\hline $\begin{array}{l}300 \\
400 \\
460 \\
584 \\
735 \\
932 \\
1866 \\
1216 \\
1255 \\
1608\end{array}$ & $\begin{array}{l}6.85(-5) \\
6.85(-4) \\
1.21(-3) \\
2.18(-3) \\
1.86(-3) \\
5.68(-4) \\
1.84(-4) \\
7.95(-5) \\
6.36(-5) \\
6.36(-6)\end{array}$ & $\begin{array}{l}6.56(-5) \\
6.56(-4) \\
1.31(-3) \\
2.41(-3) \\
1.12(-3) \\
5.71(-4) \\
2.14(-4) \\
8.85(-5) \\
7.08(-5) \\
7.08(-6)\end{array}$ & $\begin{array}{l}6.05(-5) \\
6.05(-4) \\
1.21(-3) \\
2.18(-3) \\
1.86(-3) \\
5.68(-4) \\
1.84(-4) \\
7.95(-5) \\
6.36(-5) \\
6.36(-6)\end{array}$ & $\begin{array}{l}4.81(-5) \\
4.81(-4) \\
9.63(-4) \\
1.78(-3) \\
8.48(-4) \\
4.51(-4) \\
1.51(-4) \\
6.59(-5) \\
5.27(-5) \\
5.27(-6)\end{array}$ & $\begin{array}{l}5.02(-5) \\
5.02(-4) \\
1.00(-3) \\
1.69(-3) \\
8.85(-4) \\
4.62(-4) \\
1.65(-4) \\
6.09(-5) \\
4.07(-5) \\
4.07(-6)\end{array}$ & $\begin{array}{l}4.10(-5) \\
4.18(-4) \\
8.20(-4) \\
1.33(-3) \\
6.79(-4) \\
3.43(-4) \\
1.11(-4) \\
6.18(-5) \\
4.94(-5) \\
4.94(-6)\end{array}$ & $\begin{array}{l}3.49(-5) \\
3.49(-4) \\
6.98(-4) \\
1.48(-3) \\
9.58(-4) \\
3.82(-4) \\
1.08(-4) \\
5.32(-5) \\
4.25(-5) \\
4.25(-6)\end{array}$ & $\begin{array}{l}7.26(-5) \\
7.26(-4) \\
1.45(-3) \\
2.39(-3) \\
1.41(-3) \\
5.22(-4) \\
1.92(-4) \\
4.15(-5) \\
3.32(-5) \\
3.32(-6)\end{array}$ \\
\hline
\end{tabular}

\begin{tabular}{|c|c|c|c|c|c|c|}
\hline AMBDA & 17 & 18 & 19 & 20 & 21 & 22 \\
\hline $\begin{array}{l}380 \\
480 \\
468 \\
584 \\
735 \\
932 \\
1066 \\
1216 \\
1255 \\
1688\end{array}$ & $\begin{array}{l}8.01(-5) \\
8.01(-4) \\
1.68(-3) \\
3.42(-3) \\
1.72(-3) \\
8.99(-4) \\
3.06(-4) \\
1.15(-4) \\
9.26(-5) \\
9.26(-6)\end{array}$ & $\begin{array}{l}6.36(-5) \\
6.36(-4) \\
1.27(-3) \\
3.01(-3) \\
1.32(-3) \\
8.20(-4) \\
2.98(-4) \\
1.09(-4) \\
8.77(-5) \\
\text { 1.77(-6) }\end{array}$ & $\begin{array}{l}4.23(-5) \\
4.23(-4) \\
8.47(-4) \\
2.65(-3) \\
9.66(-4) \\
4.82(-4) \\
2.10(-4) \\
1.16(-4) \\
9.31(-5) \\
9.31(-6)\end{array}$ & $\begin{array}{l}4.49(-5) \\
4.49(-4) \\
8.99(-4) \\
2.36(-3) \\
1.04(-3) \\
5.12(-4) \\
1.73(-4) \\
8.11(-5) \\
6.49(-5) \\
6.49(-6)\end{array}$ & $\begin{array}{l}3.37(-5) \\
3.37(-4) \\
6.75(-4) \\
2.28(-3) \\
1.01(-3) \\
6.69(-4) \\
2.81(-4) \\
9.52(-5) \\
7.62(-5) \\
7.62(-6)\end{array}$ & $\begin{array}{l}2.49(-5) \\
2.49(-4) \\
4.99(-4) \\
2.86(-3) \\
9.48(-4) \\
6.56(-4) \\
1.89(-4) \\
9.76(-5) \\
7.81(-5) \\
7.81(-6)\end{array}$ \\
\hline
\end{tabular}




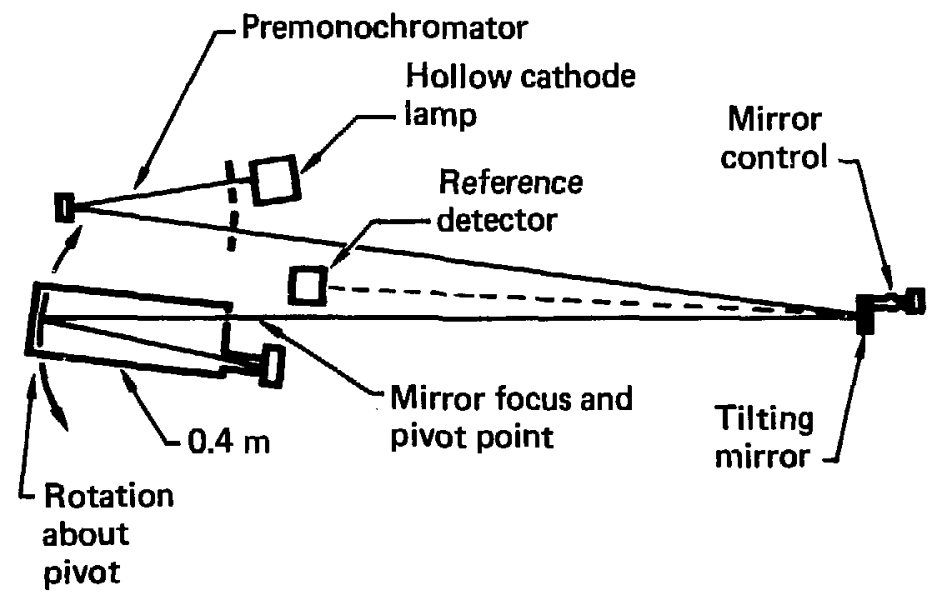

Fig. C.11. Schematic diagram of the extreme ultra-violet calibration equipment (ECE) at Johins Hopkins University. The reference diode has been calibrated at the National Bureau of Standards [48]. 
where the entrance slit of the test instrument can be placed. Care must be taken to assure that all the light monitored by the diode is allowed to pass through the instrument. The mirror is baffled to $f / 40$ so that the beam does not overfill the optics in the instrument to be calibrated. The only requirement with this arrangement is a photon source which is constant in time since the flux monitored by the reference detector is the same as that which enters the test instrument. The procedure is to measure the photon flux by directing the beam onto the diode, then tilt the mirror to direct the beam into the test instrument and take a reading, then remeasure the flux with the diode to ascertain that no drift in signal had occurred during the measurement. 


\section{REFERENCES}

[1] Coensgen, F. H. et al., Nuclear Fusion Supplement, Part I, 125 (1962).

[2] Coensgen, F. H., TMX Major Project Proposal, Lawrence Livermore National Laboratory, L.LL-PROP-148.

[3] Coensgen, F. H. et al., Phys. Rev. Lett. 44, 1132 (1980).

[4] Corre11, D. L. et al., "Ambipolar Potential Formation and Axial Confinement in TMX, " UCRL-86329, submitted to Nuclear Fusion (July 29, 1981).

[5] Chen, F. F., Introduction to Plasma Physics, Plenum Press, New York (1974).

[6] Pastukov, V. P., Nuc lear Fusion 14, 3 (1974).

[7] Coensgen, F. H. et al., Plasma Physics and Controlled Nuclear Fusion Research (Proc. 6th Int. Conf., Berchtesgaden, Germany) 33, 135 (1976).

[8] Spitzer Jr., Lyman, Physics of Fuliy Ionized Gases, Second Edition, Interscience Publishers, John Wiley and Sons, New York (1967).

[9] Baldwin, D. E., Logan, B. G., Phys. Rev. Lett. 43, 1318 (29 0ct. 1979).

[10] Fortner, R. J., private communication (1981).

[11] Drake, R. P. et al., Nuclear Fusion 21, 359 (1981).

[12] Coensgen, F. H. Simonen, T. C., Chargin, A. K., Logan, B. G., TMX Upgrade Major Project Proposal, Lawrence Livermore National Laboratory, LLL-Prop-172.

[13] Baldwin, D. E., Logan, B. G., Simonen, T. C., Physics Basis for MFTF-B, Lawrence Livermore Natiorial Laboratory, UCID-18496 (unpublished).

[14] Griem, H. R., Plasma Spectroscopy, McGraw-Hi11, New York, 176 (1964).

[15] Allen, S. L., Ph.D. thesis, The Johns Hopkins University (1980).

[16] Drake, R. P., Extreme Ultraviolet Diagnosis of a Neutral-Beam-Heated Mirror Mar.hine, Lawrence Livermore National Laboratory, UCRL-52751 (1980).

[17] Terry, J. L., Ph.D. Thesis, The Johns Hopkins University (1978) .

[18] Moos, H. W., Chen, K. I., Terry, J. L., Fastie, W. G., Appl. Opt., 12, 1611 (1979).

[19] Richards, R. K., Rev. Sci. Inst. 49, 8 (1978).

[20] Richards, R. K., Moos, H. W., Allen, S. L., Rev. Sci. Inst, 5l, i (1980).

[21] Fortner, R. J., J. Bu11. Am. Phys. Soc. 25, 881 (1980).

[22] Turner, W. C., Molvik, A. W., Williams, J., Bull. Am. Phys. Soc. 21 (1976) 485.

[23] Breton, C., DeMichelis, C., Mattioli, M., J. Quant. Spect. Rad. Trans. 19 (1978) 367. 
[24] Equipe TFR, Nuclear Fusion 15 (1975) 1053.

[25] Aymar, M., Physica (Utr.) 74 (1974) 205.

[26] Wiese, W. L., Smith, M. W., Miles, B. M., Atomic Transition

Probabilities - Sodium Through Calcium, Vol. II, U.S. Dept. of Commerce, National Bureau of Standards, NSRDS-NBS-22 (1969).

[27] Davis, J., Kepple, P. C., Blaha, M., J. Quant. Spect. Rad. Trans. 16 (1976) 1043.

[28] Van Regemorter, H., Astrophys. J. 136, 912 (1962).

[29] Hulse, R. A., private communication, Aur. 1981.

[30] Breton, C., DeMichelis, C., Finkenthal, M., Mattioli, M., CEA Euratom, EUR-CEA-FC-948 (March 1978).

[31] Hulse, R. A., Post, D. E., Mikkelsen, D. R., J. Phys. B.: Atomic Molec. Phys. 13 (1980) 3895 .

[32] Post, D. E., Jensen, R. V., Atomic Data and Nuclear Data Tables 20 (1977) 397.

[33] Drake, R. P., Moos, H. W., Nuclear Fusion 19 (1979) 407.

[34] Marmar, E. S. et a1., Nuclear Fusion 19 (1979) 485.

[35] Jolly, J., Parkinson, J., Swan, D., 8th Symposium on Engineering Problems of Fusion Research, UCRL-82886 (Nov. 12, 1979).

[36] Rognlien, T. D., Cutler, T. A., Nuclear Fusion 20 (1980) 1003.

[37] Book, D. L., NRL Plasma Formulary (Revised), Naval Research Laboratory Memorandum Report No. 3332.

[38] Hallock, G. A., Ph.D. thesis, Rensselaer Polytechnic Institute (1981).

[39] Merts, A. L., Mann, J. B., Robb, W. D., Magee, Jr., H. H., Electron Excitation Collision Strengths for Positve Atomic Ions: A Collection of Theoretical Data, Los Alamos Scientific Laboratory, LA-8267-MS (1980).

[40] Gabriel, A., Jordan, C., from Case Studies in Atomic Collision Physics, Vol. 2, Eds. E. McDaniel and M. McDowe11, North Holland, Amsterdam, 209 (1971).

[41] Wiese, W. L., Smith, M. W., Glennon, B. M., Atomic Transition Probabilities - Hydrogen Through Neon, Vol. I, U.S. Dept. of Commerce, National Bureau of Standards, NSRDS-NBS-4 (1966).

[42] Davis, J., J. Quant. Spect. Rad. Trans. 14, 549 (1974).

[43] Barnett, E. F., et al., Atomic Data for Controlled Fusion Research, Dak Ridge National Laboratory, ORNL-5207 (1977).

[44] Lotz, W., Z. Physik 206, 205 (1967).

[45] Cranda11, D. H., Physica Scripta 23, 153 (1981). 
[46] Mattioli, M., CEA Euratom, EUR-CEA-FC-761 (February 1975).

[47] Chen, K. I., Ph.D. Thesis, The Johns Hopk ins University (1979).

[48] Canfield, L. R., Johnson, R. G., Madden, R. P., App1. Opt. 12 (1973) 1611. 